\title{
Fiber-Optics Based Pressure and Temperature Sensors for Harsh Environments
}

\author{
by \\ Jason C. Twedt
}

A Thesis submitted to the Faculty of Virginia Polytechnic Institute and State University in partial fulfillment of the requirements for the degree of

\author{
MASTER OF SCIENCE \\ in \\ Aerospace Engineering \\ APPROVED \\ Rakesh K. Kapania, Chair \\ Joseph A. Schetz \\ Mayuresh Patil \\ May 18, 2007 \\ Blacksburg, Virginia
}

Keywords: Fiber optics, Pressure, Temperature, Sensor, Elliptical diaphragm, Harsh environments, Finite element analysis, Bellows, Diaphragm Sensors copyright (C)Jason Twedt 


\title{
Fiber-Optics Based Pressure and Temperature Sensors for Harsh Environments
}

\author{
Jason Twedt
}

\section{$\underline{\text { Abstract }}$}

Monitoring accurate temperature and pressure profiles in harsh environments is currently in high demand in aerospace gas turbine engines and nuclear reactor simulators. Having the ability to measure both quantities continuously over a region, without thermal coupling, using a sensor with a small size (envelope) is also highly desirable. Currently available MEMS (microelectromechanical systems) provide effective small scale pressure and temperature measurement devices, however, they have only been shown to be effective up to $600^{\circ} \mathrm{C}$ and lack the ability to perform distributed measurements unless combined with fiber-optic techniques. In general, fiber-optics provide many advantages over electrical based sensors and are the ideal choice for high temperature regimes and distributed sensing. In this thesis, preliminary designs and suggested future work are presented for a sensor built within an $3.175 \mathrm{~mm}$ radius envelope and capable of distributed pressure and temperature sensing up to temperatures reaching $800^{\circ} \mathrm{C}$. Finite element analysis via ANSYS ${ }^{\circledR}$ along with analytical verification models have been used for the design evolution. Diaphragm based designs, seem to provide easy fabrication methods and good sensi- 
tivity, however, for this design to be realized at high temperature operation, a robust bonding method must be chosen to avoid unwanted deformation due to misfit strains. 


\section{Acknowledgments}

I would like to express my sincere appreciation to Dr. Rakesh K. Kapania, and Dr. Joseph A. Schetz for their continual help, support, patience, and guidance throughout my stay here. I am so grateful for the opportunity to come and study here at Virginia Tech and work under both of these distinguished professors. Thank you both for this opportunity. I would also like to thank Dr. Mayuresh Patil for serving on my committee and for his excellence in teaching the Structural Dynamics course and also to Luna Innovations for their expertise and access to their facilities. My colleagues for creating a friendly working environment and for all their help and

support. My mother, father and extended family for all of their encouragement. Most of all, to my finacé for her understanding and supportive backing. 


\section{Contents}

1 Introduction 1

1.1 Overview. . . . . . . . . . . . . . . . . . . 1

1.2 Goal . . . . . . . . . . . . . . . . . . . 3

1.3 An Overview of Pressure and Temperature Sensors . . . . . . . . 4

1.3 .1 Pressure Sensors . . . . . . . . . . . . . . . . 4

1.3.2 Pressure Measurement Methods Review . . . . . . . . . 5

1.3.3 Temperature Measurement Method Review . . . . . . . . . . 11

1.3.4 An Overview of Fiber-Optic Methods and Applications to Pressure and Temperature Measurement . . . . . . . . . . 15

1.3.5 Development of High Temperature Sensors . . . . . . . . . 23

1.4 Organization of the Thesis $\ldots \ldots \ldots \ldots \ldots \ldots$

2 Evolution of Sensor Design $\quad 27$ 
2.1 Introduction, External Cavity Design _ . . . . . . . . . . 27

2.1.1 Mathematical Model, External Cavity Design . . . . . . . 28

2.1.2 ANSYS Simulation, External Cavity Design _ . . . . . . . 31

2.2 The Bellows Approach . . . . . . . . . . . . . . . . 35

2.2.1 ANSYS Simulation, Bellows Design . . . . . . . . . . 36

2.2.2 Mathematical Model, Bellows Design . . . . . . . . . . . . 40

2.2.3 Fabrication and Preliminary Testing Results, Bellows Design . 43

2.2.4 Conclusions, Bellows Design . . . . . . . . . . . . . . 46

3 Diaphragm Design 48

3.1 Motivation . . . . . . . . . . . . . . . . . 48

3.2 Brief Review of Nonlinear Finite Element Analysis and Shell Elements 53

3.2 .1 Nonlinearities in Structures _. . . . . . . . . . . . 53

3.2 .2 Shell Elements in ANSYS . . . . . . . . . . . . . . . 55

3.3 Deflection of an Elliptical Plate . . . . . . . . . . . . 56

3.3.1 Small-Deflection Theory . . . . . . . . . . . . . . . 57

3.3.2 Large Deformation Theory . . . . . . . . . . . . . . . . . 61

3.4 Development of a Finite Element Model for the Diaphragm Pressure

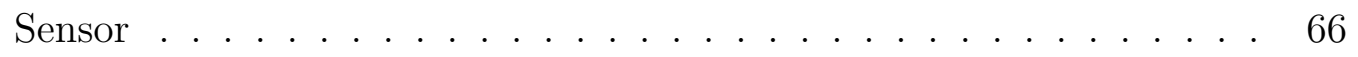

3.4.1 Verification of the Finite Element Model . . . . . . . . . 68 
3.5 Optimizing Diaphragm Geometry . . . . . . . . . . . . . 68

3.6 Summary and Conclusions . . . . . . . . . . . . . . . 73

4 Numerical Simulation of Diaphragm Design $\quad 75$

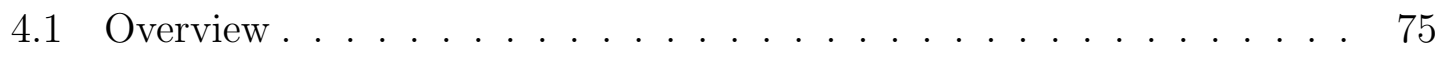

4.2 Square Tube Diaphragm Design Finite Element Model . . . . . . . . 78

4.2.1 Finite Element Model Description . . . . . . . . . . . . . 78

$4.2 .2 \quad$ Finite Element Results . . . . . . . . . . . . . . . . . . . . . 82

4.3 Square Tube Diaphragm Design Preliminary Testing Results . . . . . 88

5 Conclusions and Future Work $\quad 89$

5.1 Discussion . . . . . . . . . . . . . . . . . . . . . . . . . . 89

5.2 Future Work . . . . . . . . . . . . . . . . . 92

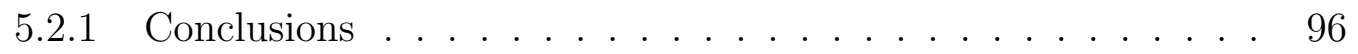

$\begin{array}{ll}\text { Bibliography } & 103\end{array}$

$\begin{array}{ll}\text { Appendices } & 110\end{array}$

A Assembly Details of Bellows Design 110

B Bellows Testing Procedure $\quad 114$ 
C Brief Review of the Structural Concepts Used in Ch. 2

C.1 Strain ........................ . . . . . . . . . . . . . .

C.1.1 A Note About the Compatibility Equations . . . . . . . . . . 118

C.2 The Finite Element Method . . . . . . . . . . . . . . . . . 118

C.2.1 Development of the Weak Form . . . . . . . . . . . . . . . . 120

C.2.2 Assembly of Finite Elements . . . . . . . . . . . . . . . . 122

D Files used in computations

127 


\section{List of Figures}

2.1 Geometry of glass sleeve fused onto a fiber . . . . . . . . . . . . . . 27

2.2 Lame's Problem . . . . . . . . . . . . . . . . . . . . . . . . 29

2.3 Axisymmetric Loading and Boundary Conditions for Fused Capillary Tube Design ....................... 33

2.4 Axial Strain Through Fiber Core From ANSYS Simulation Results, starting from $\mathrm{y}=0 \mathrm{~mm}$ to $\mathrm{y}=5 \mathrm{~mm} \ldots \ldots . . . . . . . .34$

2.5 The Final Sensor Design with Bellows (Dimensions are in inches, 1 inch $=2.54 \mathrm{~cm}) \ldots \ldots \ldots \ldots$

2.6 ANSYS Geometry and Loading for Bellows Design . . . . . . . . . . 38

2.7 Axial Strain Simulation Results Through Core of Fiber, Bellows Design 39

2.8 Bellows Spring Model . . . . . . . . . . . . . . . . . . . . 40

2.9 Reduced Bellows Spring Model . . . . . . . . . . . . . . . . . . . . . 41

2.10 The Prototype After Fabrication . . . . . . . . . . . . . . . . 43 
2.11 Preliminary Testing Results _. . . . . . . . . . . . . . . . 44

2.12 Numerical vs. Experimental Strain Results . . . . . . . . . . . . 45

3.1 Luna Energy Diaphragm Sensor Concept . . . . . . . . . . . . . 50

3.2 Notches Milled into a Circular Tube, Forming an Elliptical Diaphragm 50

3.3 ANSYS model of a pressure sensor formed from a $3.175 \mathrm{~mm}(0.125 ")$ diameter stainless steel tube with a $0.7874 \mathrm{~mm}(0.031$ ") wall thickness and a circular notch $\left(\mathrm{r}=0.7112 \mathrm{~mm}\left(0.028^{\prime \prime}\right)\right)$, resulting in a minimum thickness of $0.0762 \mathrm{~mm}(0.003$ "). See Fig. 3.2 for a more conceptual sketch of the sensor. Applied pressure, $2.413 \mathrm{MPa}(350 \mathrm{psi}) \ldots \ldots . .51$

3.4 Square Tube with Machined Diaphragm _. . . . . . . . . . . . 52

3.5 Structural nonlinearites . . . . . . . . . . . . . . . . . . . 54

3.6 Nash and Cooley dimensionless deflection versus load results as compared to linear deformation theory $\ldots \ldots \ldots \ldots$. . . . . 65

3.7 Diaphragm and loading conditions created by input file . . . . . . 67

3.8 Verification of input file with analytical results . . . . . . . . . . . 69

3.9 Axial Strain distribution for varying lengths . . . . . . . . . . 72

3.10 Axial Strain distribution for a square plate under uniform pressure . . 73

4.1 Silver plate bonding technique . . . . . . . . . . . . 76

4.2 Silver hemisphere bonding technique . . . . . . . . . . 77 
4.3 Loading and boundary conditions for square tube mill design . . . . . 79

4.4 Contact regions between parts . . . . . . . . . . . . . . . . . 83

4.5 ANSYS axial strain results for a plate bonded structure using method two, Applied Pressure: $2.413 \mathrm{MPa}[350 \mathrm{psi}]$. . . . . . . . . . . . . . 84

4.6 ANSYS axial strain results for a hemisphere bonded structure using method two, Applied Pressure: $2.413 \mathrm{MPa}[350 \mathrm{psi}]$. . . . . . . . . 85

4.7 ANSYS axial strain results for a plate bonded structure using method one, Applied Pressure: $2.413 \mathrm{MPa}[350 \mathrm{psi}]$. . . . . . . . . . . . . . 86

4.8 Prototype, diaphragm design, after fabrication. Face opposite to diaphragm was removed for the bonding of the fiber. A back cover was used to seal the opening. . . . . . . . . . . . . . . . 88

5.1 Simple finite element model for thermoelastic response of a bi-layer . 94

5.2 Resulting deformation and axial strain state from model described in

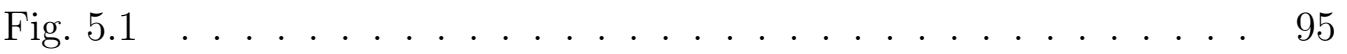

5.3 Material Search (Metals), Maximum Service Temperature vs. CTE . 98

5.4 Material Search (Metals), Figure 5.3 zoomed in, with color rendering turned off. . . . . . . . . . . . . . . . . . . . . 99

5.5 Material Search (Ceramic and glasses), Maximum Service Temperature vs. CTE . . . . . . . . . . . . . . . . 102 
A.1 Detailed Drawing of the Hub . . . . . . . . . . . . 111

A.2 Detailed Drawing of the Bellows . . . . . . . . . . . . . . . . 112 


\section{List of Tables}

2.1 Summary of Simulation Results . . . . . . . . . . . . . . . 35

2.2 Material Properties, Bellows Model . . . . . . . . . . . . . . 37

3.1 Summary of Axial Strain Results from Simulation . . . . . . . . . 70

3.2 Summary of Transverse Strain Results from Simulation . . . . . . . 71

4.1 Material Properties, Diaphragm Model . . . . . . . . . . . . . . . 81

5.1 Material Properties . . . . . . . . . . . . . . . . . . . . 100 


\section{Chapter 1}

\section{Introduction}

\subsection{Overview}

Accurate measurement of pressure and temperature has been of great importance to our society's development. Consider for instance, the aerospace, automotive, oil drilling and medical fields. Developments of highly accurate, reliable pressure and temperature sensors, have provided many benefits to society today. Betterments include safety in travel, by means of both ground and air, as well as human longevity. Several recent pressure and temperature devices are available today that enable us to increase our quality of life.

The engineering concepts for measuring pressure and temperature are relatively 
simple, as will be shown, however the operational environment has become more demanding, pushing researchers to develop sensors that are capable of high and low temperature regimes while keeping their dimensions small. Consider the following examples of various sensing environments. Palmer et al. [1], demonstrate the introduction of a fiber-optic based sensor into an aerospace gas turbine to monitor the dynamic pressure fluctuations for the purpose of a more efficient, lower emission engine. The sensor is capable of measuring pressure of 500 psig at $1922^{\circ} \mathrm{F}$. The automotive industry uses temperature and pressure sensors for engine, fuel systems, and tire monitoring. Temperature regimes in this industry ranges from $-40^{\circ} \mathrm{C}$ to $125^{\circ} \mathrm{C}[2]$. Accurate pressure measuring devices are a necessity for the Deep Draft Caisson Vessel, a 75 story tall, moored oil rig in $4,800 \mathrm{ft}$. of water [3]. It needs to operate at low temperatures and extremely high pressures. Micro-scale sensors are available in the medical field. An implanted pressure sensor in the heart has been designed to monitor the pressure in the heart for patients suffering from congestive heart failure. The implanted pressure sensor is a deflection based pressure sensor the size of a grain of rice [4]. 


\subsection{Goal}

Monitoring accurate temperature and pressure profiles in harsh environments is currently in high demand in aerospace, gas turbine engines and nuclear reactor simulators. Having the ability to measure both quantities continuously over a region, without thermal coupling, and within a small envelope is also highly desirable. The work performed in this thesis is for the Safe Affordable Fission Engine (SAFE-100), a simulator being developed to supplement the introduction of early flight fission. The SAFE-100 is located at the Early Flight Fission Test Facility of the NASA Marshall Space Flight Center. It provides the capability to simulate the heat of fission in an electrically heated nuclear reactor thermal simulator. The idea is, that by properly characterizing the thermo-mechanical activity within non-nuclear reactor simulators, nuclear operation can be more accurately controlled and confidence in thermo-mechanical simulations will be high. However, the ability to characterize non-nuclear test core simulators is currently limited by the lack of instrumentation options available for distributing large numbers of sensors throughout the core to monitor these parameters in real-time. Resistance temperature detectors (RTDs) and thermocouples provide only single point measurements and, because of logistical problems associated with limited physical accessibility, cannot be used in any significant numbers. To address this need, we have proposed to develop a fiber-optic 
instrumentation system based on Luna Innovations' unique distributed sensing technology, which uses fiber Bragg gratings or special fiber as the sensing transducers for temperature, strain, and pressure measurements. The end result of this research is an advanced instrumentation system capable of monitoring multiplexed fiber-optic sensor arrays that are capable of high-temperature operation.

\subsection{An Overview of Pressure and Temperature Sensors}

\subsubsection{Pressure Sensors}

Pressure is defined as the force per unit area applied perpendicular to a surface.

Pressure is typically expressed in many different units depending on one's preference.

Here are some conversions that may help in dealing with these units:

$$
1 \mathrm{~atm}=14.70 \mathrm{psi}=1.013 \mathrm{E} 05 \mathrm{~Pa}=1.013 \mathrm{bar}=760 \mathrm{torr}
$$

Pressure is measured relative to a reference pressure. There are different terms used to describe pressure measurement, each of which differ only by the reference pressure. 


\section{Absolute Pressure}

Absolute pressure is measured with reference to a perfect vacuum. Thus to make an absolute pressure gage a reference vacuum must be sealed within the device.

\section{Gage Pressure}

Gage pressure is measured relative to the ambient atmospheric pressure. Atmospheric pressure varies with altitude above sea level and is a result of the weight of air in the Earth's atmosphere.

\section{Differential Pressure}

Differential pressure measures one pressure relative to another. Gage pressure is a type of differential pressure where one pressure is always the atmospheric pressure.

\subsubsection{Pressure Measurement Methods Review}

Pressure detection methods can be divided into two groups [5] - techniques that directly measure the pressure applied to a surface area and indirectly by use of a transducer (converts one type of energy to another allowing pressure measurement). An example of the former is a simple pressure manometer while indirect measurements can be obtained through mechanical deflection or various electrical techniques. 
These methods will be described in more detail in the following paragraphs.

\section{Manometer}

This popular pressure measuring device dates back to the mid-1600's [6]. This device measures pressures through change in height of a column of fluid. Different liquids with varying specific gravities can be used depending on the desired pressure range. The biggest drawback to this method is the fluid's density dependence on temperature, so at higher/lower temperatures, errors can be induced into the pressure measurement. For this reason manometers are typically used as a standard to calibrate other pressure devices.

\section{Mechanical Pressure Measuring Devices}

An example of a mechanical pressure sensor is a bellows gage. This is a simple device that will work well at low pressures. A bellows, thin walled metal device with convolutions, is essentially a spring with stiffness dependent on its outside diameter, thickness, and number of convolutions [6]. As pressure changes, the bellows will extend or contract, moving a calibrated needle arm revealing the pressure.

Bourdon Tube elements, patented by Eugene Bourdon in 1849 is another example of a mechanical element used to measure pressure. Bourdon elements are made of elastic materials and shaped into a number of different configurations, for instance, a 
spiral tube or C-shaped tube. While pressure is applied to one end of the shaped tube it tends to straighten out. This movement on the other end of the tube is detected by a pointer over a calibrated dial. The deflection of each tube is dependent on the material properties of the tube, its dimensions, and curvature.

Mechanical devices are typically good for relatively low pressure measurements. Typically the accuracy of a mechanical gage is $2.0 \%$ of full scale and can be refined in some cases to $0.1 \%$ full scale [7]. The culprit for these errors lies in the devices' flexing element, both hysteresis and temperature dependent properties attribute to error in the system.

\section{Electrical Transducers}

Electronic methods of pressure measurement greatly improved the accuracy and reliability of mechanical transducers. Electronic transducers are devices that convert mechanical deflection (strain) into an electrical signal. These types of transducers offer many advantages over mechanical pressure sensors. For example, electric devices provide the ability to transmit data over long distance while offering ways to compensate for nonlinear material effects. Years of research and development has lead to a variety of electrical pressure transducers:

- Piezoresistive 


\section{- Capacitive}

- Strain-Gage

- Piezoelectric

- Linear Variable Differential Transformers (LVDTs)

All of the above transducers convert a mechanical deflection, due to external pressure, into an electrical signal. Each method varies in its overall performance and thus its typical application. Capacitive transducers for instance have a pressure range from one inch of water to 5000 psi, full scale, with accuracies of 0.1 to $0.5 \%$ [6]. Piezoelectric sensors on the other hand are known for their very high natural frequency and low noise, making these sensors suitable for measuring very fast pressure changes, such as in shock waves [6].

MEMS (microelectromechanical systems), have been brought about due to the success in the semiconductor industry and are typically silicon based devices. Silicon is the second most common element on earth making for a low cost and ideal material for large batch production. The other advantage of silicon based devices is silicon's single-crystal structure, which allows for high strength and virtually no hysteresis [6]. Highly precise micromachining allows for dimensions as low as a few microns resulting in a highly sensitive device. The process of micromachining involves various 
steps such as photolithography and silicon etching.

Silicon etching originally was classified as isotropic or anisotropic and was accomplished by either wet chemical or dry etching techniques. Isotropic techniques have no directional preference while anisotropic etching takes advantage of etch rate in different directions, due to the crystallographic orientation of the material, resulting in more precise cuts. More recently, highly directional plasma etching techniques have been developed allowing for faster etch rates [8]. This dry etching technique uses high density sulfur hexafluoride and oxygen plasma. Generally the sensors are fabricated by sealing two wafers together.

MEMS based pressure sensors are generally piezoresistive or capacitive. In the former, strain-sensitive resistors are implanted into silicon wafers and connected to a Wheatstone bridge configuration. A diaphragm is etched on the other side, as pressure is applied, the resistors are strained causing an imbalance in the Wheatstone bridge proportional to the pressure. A capacitive pressure transducer uses deflection of a thin micromachined silicon diaphragm, due to the onset of pressure, to change the distance between the plates of a parallel-plate capacitor, thus changing its capacitance proportionally. Through two electrical wires the capacitor is connected to a circuit, designed to convert capacitance into a voltage or frequency signal for sensor read out [6]. 
Today most commercial MEMS pressure sensors are of the piezoresistive type due to their inherent strong linearity of pressure and resistance. However, their major drawbacks are in their cross-sensitivity to temperature and large power consumption. These sensors can drift 100 Pa per day due to temperature coupling effects, making them inadequate for long term measurements [9]. In contrast, capacitive MEMS pressure sensors offer some alleviation to above stated problems of piezoresistive sensors, while being supreme in sensitivity. Though there exists a nonlinear capacitancepressure relation, the deflection basis device is less sensitive to temperature variation [10] and require less power. But, as only small magnitudes of capacitance occur, outside noise can affect the signal requiring short well placed wires, thus making packaging an issue.

Though MEMS systems are small and highly sensitive and offer low cost manufacturing, they are limited in their application at high temperatures. Silicon based MEMS systems are only capable of operating at $150^{\circ} \mathrm{C}[11,12]$. Much research has been conducted in developing high temperature pressure sensors using MEMS devices and will be discussed later. One overlying problem with all electronic based sensors is their sensitivity to EMI (electromagnetic interference), a problem not faced by fiber-optic based sensors. 


\subsubsection{Temperature Measurement Method Review}

The definition of temperature was stated by James Clerk Maxwell as: temperature of a body is its thermal state, regarded as a measure of its ability to transfer heat to other bodies [13]. Today, the three most common temperature scales are the Kelvin scale, Celsius Scale and the Fahrenheit scale where temperature is denoted by ${ }^{\circ} \mathrm{K}$, ${ }^{\circ} \mathrm{C}$, and ${ }^{\circ} \mathrm{F}$ respectively. The relation between temperature scales are given by:

$$
\begin{aligned}
K & =t_{c}+273.15 \\
{ }^{\circ} F & =\frac{9}{5} t_{c}+32
\end{aligned}
$$

where $t_{c}$ is the temperature in degrees Celsius. Temperature sensors can most generally be catorigized into three types, non-electric, electric, and fiber-optic. Examples of these types of thermometers will be discussed in the following paragraphs.

\section{Non-Electric Thermometers}

Non-electric thermometers encapsulate temperature measuring devices that are based on physical properties of either a solid, liquid or gas. Liquid-in-gas thermometers is one such case that has a working principal that is based on temperature dependent volume properties of the liquid, used in a capillary tube. This effect is governed by 
the following relation:

$$
\Delta V=V \beta_{a} \Delta T
$$

Here, it is assumed that the bulb volume, $V_{b}$, is much greater than the liquid contained in the capillary tube of volume $V . \beta_{a}$ is defined as the average apparent coefficient of cubic thermal expansion of the liquid used, $\Delta V$ represents the change in volume of the liquid due to temperature variation $\Delta T . \beta_{a}$ is a constant defined as the difference between the cubic coefficients of thermal expansion between the liquid and glass, $\beta_{a}=\beta_{l}-\beta_{g}$. From the above information an expression for the thermometer sensitivity of liquid filled bulb connected to a thin capillary tube of diameter $d$ can be found out.

$$
\frac{\Delta l}{\Delta T}=\frac{4 V_{b}\left(\beta_{a}\right)}{\pi d^{2}}
$$

It can be noticed, that the sensitivity increases proportionally with the bulb volume, the coefficient of cubic expansion, and the inverse square of the diameter of the capillary. Thus for high sensitivity, long thin tubes are necessary. This poses a problem due to the frailty of the capillary tube and its tendency to break due to surface tension and rough handling, which is why this design is limited in its applications.

The expansion of solids is temperature dependent, this is another physical property used to estimate temperature. Dilation thermometers are a class of thermome- 
ters that work on this principal. The change in length of a composite rod, that is one part solid material with CTE, $\alpha_{1}$ and the other part a different material with CTE, $\alpha_{2}$ is given by:

$$
\Delta l=l\left(\alpha_{1}-\alpha_{2}\right) \Delta T
$$

Because this change in length is small, the effect is normally mechanically amplified. Dilation thermometers can measure temperatures below $1000^{\circ} \mathrm{C}$ with errors of $\pm 1 \%$ $\pm 2 \%[13]$

\section{Electric Based Thermometers}

In today's industry, there are two predominant types of electrical based thermometers: thermoelectric, and resistance thermometers. In 1821, T. Seebeck observed current flow through two different metals connected in a closed loop when their junctions were subjected to a thermal gradient. He described this as thermoelectric force given by:

$$
E_{A}\left(t_{1}, t_{2}\right)=\int_{t_{2}}^{t_{1}} \sigma_{A}(t) d t
$$

where $\sigma_{A}$ is the materials Seebeck coefficient. Thermocouples are designed around Seebeck's phenomenon, comprised of two dissimilar conductors connected together at one end; will create a current when their junction is subject to a change in temperature. Some examples of an effective pair of conducting materials are as follows; 
the platinum-rhodium/platinum, code $\mathrm{S}$, is a popular rare-metal thermocouple that is specified for normal continuous use in the range $-50^{\circ} \mathrm{C}$ to $1300^{\circ} \mathrm{C}$ and can be extended to $1600^{\circ} \mathrm{C}$ for short term readings. Alternatively, nickel-chromium/nickelaluminum, code $\mathrm{K}$, is the most commonly used base-metal type thermocouple. Its application ranges from $-200^{\circ} \mathrm{C}$ to $1100^{\circ} \mathrm{C}$, extending to $1300^{\circ} \mathrm{C}$ for short term measurements. Though, this type of thermocouple has a problem with hysteresis at temperatures above $500^{\circ} \mathrm{C}[13]$. Here code $\mathrm{S} / \mathrm{T}$ indicate the type of method used to permanently connect the conductors at their measuring junctions. For instance, in a K-type thermocouple construction, both conductors are twisted together and subsequently welded in non-reducing oxyacetylene flame. Although thermocouples are very durable up to large temperatures their main limitation is accuracy, errors less than $1^{\circ} \mathrm{C}$ can be difficult to achieve.

Resistance temperature detectors (RTDs) are another class of electric type thermometers. RTDs are known as a modulating sensor because they require the supply of energy to support temperature measurement [13]. These devices work on the basis of temperature dependence of resistance for metal conductors. RTD's are capable of operating between $-260^{\circ} \mathrm{C}$ and $700^{\circ} \mathrm{C}$ with good repeatability and stability [13] These high accuracy devices are suitable for precision applications under $600^{\circ} \mathrm{C}$.

In summary, research in electric based thermometers has resulted in high temper- 
ature sustaining, good stability devices. However, these devices can only give a single

point measurement. In contrast, an array of connected sensors can give a profile of the whole environment. This type of multiplexing is a highly proven method using fiber-optic Bragg grating sensors and will be the subject of the following subsections.

\subsubsection{An Overview of Fiber-Optic Methods and Applica- tions to Pressure and Temperature Measurement}

Fiber optic sensors hold many advantages over other sensing methods, for instance they are, nonelectrical, small in size and weight, immune to radio frequency interference (RFI) and electro-magnetic interference (EMI), show high accuracy, and secure data transmission [14]. Fiber optic sensors transmit light through optical fibers and detect changes in light intensity, phase, and wavelength induced by a perturbing environment. Displacement, temperature, pressure, strain, rotation, flow, vibration, magnetic fields and electric fields are just some of the measuring capabilities of optical fibers. Krohn[14] states that there are three basic categories of fiber optic sensors: phase-modulated, intensity-modulated, and wavelength-modulated, all of which are briefly introduced below. 


\section{Intensity-Modulated Fiber Optic Sensors}

This type of sensor generally measures displacement or some other physical perturbation with the fiber or mechanical transducer by detecting the amount of light that has been lost due to the perturbing environment. There are three general concepts involved in intensity modulation: transmission, reflection, and microbending.

Transmission is a relatively simple concept, light travels through an optical probe exits into a medium and then enters another fiber optic probe where light intensity is measured. The light intensity follows the $1 / r^{2}$ law, where $\mathrm{r}$ is the distance between the probes. The reflective concept is comprised of a pair of single fibers or two bundles of fibers, one bundle/fiber transmits light onto a reflecting target at some distance away while the other bundle/fiber traps the reflected wave and transmits it to a detector. The light intensity is a function of the distance from the target. The linear slope allows potential accuracy of one millionth of an inch [14]. Microbending offers another attractive sensor concept. Here a transducer is used to bend the fiber due to a small change in some physical quantity (pressure). As the fiber is bent, light is lost through the wall of the fiber, thus the amount of received light is related to the physical property. Like reflective sensors, microbending sensors are potentially low cost and accurate. Their accuracy increases as the number of bend points increases and/or the spacing between the bend points decreases [14]. Microbend pressure 
sensors have been reported to give very high resolution (typically better than $0.1 \%$ ), but large hysteresis and the power fluctuation associated with the fiber loss limit their accuracy within a few percent of the full scale [15].

Enhanced sensitivity can be obtained by further adding absorption gratings to the fiber face or with a fixed and a rotating polarizing lens. The major problems associated with intensity modulated sensors are variations in light source intensity and microbending in the leads to and from the sensing region [14]. It is apparent that the large size of the mechanical transducer necessary for high resolution makes the microbend fiber optic pressure sensor impractical for small envelope requirements.

\section{Phase-Modulated Fiber Optic Sensors}

In these types of sensors generally light is split and injected into two separate fibers, one path remains unphased while the other fiber flows through the sensing environment. If the environment perturbs the fiber a phase shift occurs. This phase shift relative to the unphased wave can be detected very precisely using an interferometer. There are four types of interferometer configurations, Mach-Zehnder, Michelson, Fabry-Perot, and Sagnac. The Fabry-Perot scheme offers extremely high sensitivity, giving twice the sensitivity of the other techniques [14]. Phase detection works off the principal of constructive and destructive interference. When the fiber undergoes a 
change in length due to the transducing element there is an associative phase change causing the two waves in the detecting region to destructively interfere.

\section{Wavelength-Modulated Fiber Optic Sensors}

As described earlier, wavelength modulated sensors measure the change in wavelength of emitted light due to a perturbation of the surrounding environment. Bragg gratings are used to accurately calculate this change in wavelength and offer several advantages, for instance, their ability to make the wavelength shift independent of

light source. Gage lengths can be as small as $0.01 \mathrm{~mm}$, creating the ability for small sensor construction. More importantly (for this research), Bragg gratings can be used in multiplexing schemes for distributive sensing [14]. In fact, FBGs combined with a readout system based on Optical Frequency Domain Reflectometry (OFDR) have the capability to read several thousand individual sensors on a single optical fiber [16].

Bragg gratings are basically sections of index of refraction variations made into the core of a fiber through intense UV light. The change in index of refraction $(\Delta n)$ in the gratings region is typically $10^{-5}$ to $10^{-3}$. The resonant wavelength of the Bragg grating is given by:

$$
\lambda=2 n \Lambda
$$


where, $\Lambda$ is the spacing between gratings periods and $n$ in the refractive index of the core. At wavelengths other than $\lambda$, light passes through the gratings essentially unaffected, at the resonant wavelength the signal is reflected [14]. Thus the wavelength shifts can be measured by either the transmitted or reflected signal.

The index of refraction is sensitive to both change in length and temperature change. As the fiber is elongated, by a transducer or temperature change, its radial dimension decreases due to Poisson's effect causing a change in the index of refraction. The refraction index is also a temperature dependent quantity. The change in resonant Bragg wavelength, $\Delta \lambda_{B}$, is given by [17]:

$$
\Delta \lambda_{B}=2 n \Lambda\left[\left(1-\left(\frac{n^{2}}{2}\right)\left[P_{12}-\nu\left(P_{11}+P_{12}\right)\right]\right) \epsilon+\left[\alpha+n \frac{d n}{d T}\right] \Delta T\right]
$$

where:

- $\epsilon=$ the applied axial strain

- $P_{i j}=$ the stress optic coefficient

- $\alpha=$ coefficient of thermal expansion

- $\nu=$ Poisson's ratio

- $\mathrm{n}=$ the refractive index of the core of the fiber optic cable 
- $\Delta T=$ Temperature change

For silica, the term $\left(\frac{n^{2}}{2}\right)\left[P_{12}-\nu\left(P_{11}+P_{12}\right)\right]=0.22$, for constant temperature Eq. 1.9 becomes:

$$
\Delta \lambda_{B}=1.56 n \Lambda \epsilon
$$

Interferometric detection schemes are used to detect wavelength shifts in Bragg gratings [14]. Light from the Bragg grating is passed through a interferometer of differing path lengths. A phase shift associated with the Bragg gratings wavelength shift is then detected, this relationship is given by [17]:

$$
\Delta \Phi=\frac{2 \pi n d}{\lambda^{2}} \Delta \lambda
$$

In summary, optical sensors employing Bragg gratings offer multiplexing of many sensors that use the same signal processing unit along with immunity to power loss variations [9]. Early in Bragg grating development, there existed some drawbacks to using Bragg gratings and since then these problems have been mitigated.

Fiber Bragg grating properties, i.e. index of refraction, have been shown to decay with rising temperature, that is wavelength shifts become also time dependent [18]. The mechanical strength of the fiber is dependent upon the flaws (scratches) on 
its surface. In addition to the natural flaws associated with manufacturing, human interaction (handling, stripping, re coating for UV exposure) add further structural flaws and internal stresses altering the overall reliability of the fiber. However, [19] reports that higher-strength stripped and re coated fiber bragg gratings obtained through proof testing have a $99 \%$ survival probability when loaded at $1.5 \%$ static strain lifetime in excess of 25 years. [19] also shows that pre-annealing the FBG's prior to their application will reduce wavelength drift due to UV-illumination effects. Luna Innovations have shown that they can fabricate Bragg gratings that are less sensitive to temperature and radiation fading though the use of heat treatment [16]. In addition to this, their method of interrogation is considerably more sensitive than traditional Bragg measurement instruments, as much as 1000 times more sensitive, thus reducing any affects in wavelength shift due to small decay. Luna also has the capability to measure wavelength shifts using their optical backscatter reflectometer (OBR), technology based on the Rayleigh scattering concept. Rayleigh scattering is yet another way to achieve distributed sensing. In this method, the natural flaws in the fiber create a unique reflection/transmitted wavelength profile. Before each measurement is taken, a baseline profile is found. Then any change in wavelength due to a perturbing environment is compared to the baseline to correlate the measured quantity with the wavelength shift. 
Temperature discrimination from strain effects is yet another issue to be resolved for harsh environment sensing, recall Eq. 1.9. There are many approaches for doing so. One approach uses a reference grating near the measurement site isolated from strain effects, thus while one grating measures wavelength shifts due to temperature and strain, the reference grating only reads a shift due to temperature [17]. The difference is the strain alone. It is interesting to note that a bare fiber can accurately measure temperatures up to $1000^{\circ} \mathrm{C},[20]$, with $\pm 10^{\circ} \mathrm{C}$ accuracy using Rayleigh scattering. Many other approaches to temperature discrimination use two gratings, each with different responses to temperature and strain as defined by the following equation [14].

$$
\left(\frac{\Delta \lambda_{1}}{\Delta \lambda_{2}}\right)=\left(\frac{K_{\epsilon_{1}} K_{T 1}}{K_{\epsilon_{2}} K_{T 2}}\right)\left(\frac{\epsilon}{T}\right)
$$

Here, $K_{\epsilon_{i}}$ is the $i^{\text {th }}$ grating response to strain and similarly $K_{T i}$ is the $i^{t h}$ grating response to temperature. Kersey, et al. [17] achieved different responsivities by varying the cladding diameter between two gratings. Here, both gratings experience the same temperature response and the thinner of the two experiences more strain sensitivity, making it possible to subtract the temperature effect. Reekie, et al. [21] superimposed two Bragg gratings over one another, each having different resonant wavelengths achieving different responsivities and thus temperature and strain dis- 
crimination.

\subsubsection{Development of High Temperature Sensors}

As a result of growing demands for higher temperature measurements, especially in the automotive and aerospace fields, there have been numerous publications in high temperature sensing involving mostly MEMS and fiber-optic based systems.

High temperature electronic approaches have been unveiled in recent years due to the increasing demand in the aerospace, automotive and oil drilling fields. Originally, piezoresistive MEMS sensors using the silicon technology could only be useful up to $120^{\circ}-150^{\circ} \mathrm{C}$ because in this temperature range pn-junction leakage occurs $[11,12]$. Diem et al. [11] show that with the use of silicon on insulator (SOI) substrate, particularly SIMOX (separation by ion implantation of oxygen) substrates, can create highly efficient piezoresistive and capacitive surface micromachined sensors. SIMOX substrates offer a perfect insulation between the sensing element and silicon, allowing temperature operation up to $300^{\circ} \mathrm{C}$. Kasten et al.[22] demonstrated this technology. They proposed the use of capacitive, surface-micromachined, pressure sensor based on SIMOX-technology and show its ability to measure at temperatures up to $340^{\circ} \mathrm{C}$. They report that future research is being conducted to enhance the prolonged stability of the sensor. Above $500^{\circ} \mathrm{C}$, silicon's mechanical properties begin to deteriorate 
and plastic deformation sets in [23]. Kroetz et al. [12] investigate new silicon compatible material systems that will allow for even higher temperature ranges. They find that silicon carbide on SOI substrates, namely 3C-SiC on silicon surfaces provide good performance up to $500^{\circ} \mathrm{C}$. Beyond that, Ned et al. [24] introduced a $6 \mathrm{H}-\mathrm{SiC}$ piezoresistive pressure sensor operational between room temperature and $600^{\circ} \mathrm{C}$.

Fiber-optical based methods have been very successful in high temperature sensing, eclipsing the temperature ranges of MEMS based systems. Fang et al. [25] developed a fiber-optic temperature sensor capable of withstanding temperatures as high as $1500^{\circ} \mathrm{C}$ with $0.15 \%$ accuracy. The sensor uses the concept of bierefringence of a sapphire flat, which is placed at the end of a fiber. Palmer et al. [1] demonstrate the "world's highest" temperature pressure sensor based on the idea of a Fabry-Perot cavity. The prototype has shown to be capable of measuring pressure accurately at temperatures exceeding $1050^{\circ} \mathrm{C}$. They argue that because fiber-optic sensors do not rely on electrical material phenomenom and are immune to electromagnetic interference, fiber-optic sensors offer a more robust solution as compared to electronic sensing techniques.

In summary, previous literature has shown that multiplexing of sensors is best achieved by use of fiber-optic methods. Dasgupta, Scofield, and Boyd [26] proposed the development of MOEMS (microoptoelectromechanical systems) based sensors 
using wide band gap semiconductor materials that have the potential to operate in temperature environments approaching $1000^{\circ} \mathrm{C}$. In this development, they recognize the need for multiplexed arrays of a single sensor and have proposed optical multiplexed sensor methods to employ this idea.

\subsection{Organization of the Thesis}

The problem being investigated in this thesis is to successfully design and build a Distributed Pressure Sensor (DPS) capable of high temperature operation, with a target performance window of operation at $800^{\circ} \mathrm{C}$, dynamic pressure range of 0 $350 \mathrm{psi}$, and a maximum diameter of $\frac{1}{8}$ inch. These restrictions stem from NASA SAFE100 requirements. Chapter 2 will begin with background information pertaining to the structural concepts used in design. Then a description of our initial design approaches, from the conception stage to analytical models complimented with sim-

ulations from ANSYS ${ }^{\circledR}$ finite element software. The chapter will conclude with fabrication methods and preliminary test results of the bellows design sensor. Chapter 3 will discuss the motivation and development of the diaphragm based design. Verification of the design is introduced with a comparison between plate theory and ANSYS simulation results. Chapter 4 will describe the numerical simulations of the final sensor design along with experimental testing results. This thesis will conclude 
with Chapter 5, where a summary of the work performed along with future work will be included. 


\section{Chapter 2}

\section{Evolution of Sensor Design}

\subsection{Introduction, External Cavity Design}

The main objective in the sensor design was to find a way to enhance axial strain within a Corning SMD28e fiber-optic cable due to surrounding pressure. In the initial attempt, a portion of the fiber was housed in an external glass capillary tube, end caps made from fused silicon were used to create a hermetic seal, see Fig. 2.1. A

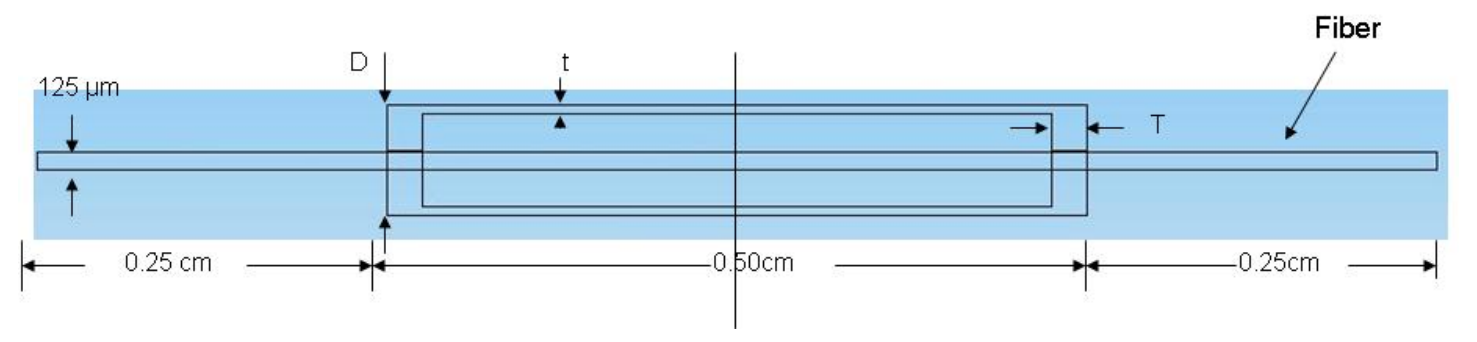

Figure 2.1: Geometry of glass sleeve fused onto a fiber 
simple analytical model was created to predict the axial strain through the center of the fiber. This development was undertaken to gain a broader insight into the parameters that would affect the strain. These results were then compared to a 2-

D axisymmetric finite element model created in ANSYS ${ }^{\circledR}$. A review of structural concepts such as strain and the finite element method are reviewed for the reader's convenience in Appendix C.

\subsubsection{Mathematical Model, External Cavity Design}

To approximate the axial strain through the core of a fiber optic cable with surrounding fused capillary tube and subjected to external pressure loading, a linear combination of tensile strain resulting from Poisson's ratio and the compressive strain due to the end pressure was assumed. Let us first, consider the tensile strain resulting from externally applied pressure to the sleeve. This is a common axisymmetric problem where stresses and strains can easily be found through the use of solution of the Lame's problem. Lame's problem involves stress calculation through the use of the Airy's stress function, $\Phi$, which is a function that implicitly satisfies the equations of equilibrium [27]. Adding together solutions to the Bi-Harmonic equation, (Eq. 2.1, Eq. 2.2) found from satisfying the compatibility conditions, and applying the boundary conditions, yields expressions for both the radial and hoop stress (Eq. 2.3 and 
Eq. 2.4) for a capillary tube with outer radius $b$, inner radius $a$ and internal pressure $P_{i}$ and external pressure $P_{o}$.

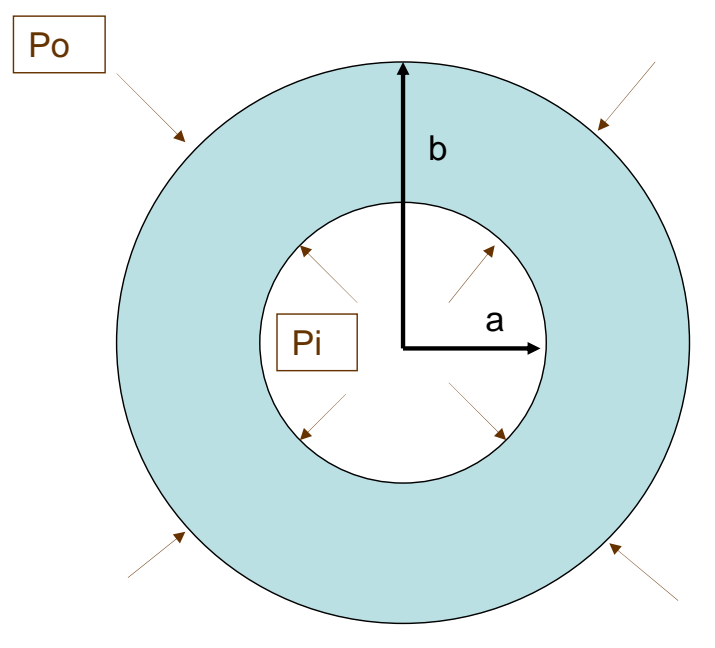

Figure 2.2: Lame's Problem 


$$
\begin{aligned}
\nabla^{4} \Phi & =0 \\
\Phi & =A r^{2}+B \log r+C r^{2} \log r+D \\
\sigma_{r r} & =\frac{a^{2} p_{i}-b^{2} p_{o}}{b^{2}-a^{2}}+\frac{1}{r^{2}}\left[\frac{a^{2} b^{2}\left(p_{0}-p_{i}\right)}{b^{2}-a^{2}}\right] \\
\sigma_{\theta \theta} & =\frac{a^{2} p_{i}-b^{2} p_{o}}{b^{2}-a^{2}}-\frac{1}{r^{2}}\left[\frac{a^{2} b^{2}\left(p_{0}-p_{i}\right)}{b^{2}-a^{2}}\right]
\end{aligned}
$$

Here, A,B,C,D are constants to be solved for upon application of the boundary conditions. We assume a plane stress condition on a very thin slice of the sleeve, and calculate the axial strain. Plane stress implies, $\sigma_{z z}=0$, and the stress strain relationship in the axial direction reduces to:

$$
\epsilon_{z z_{P}}=-\frac{\nu}{E}\left(\sigma_{r r}+\sigma_{\theta \theta}\right)
$$

The axial compressive strain due to the end force is simply calculated form Hooke's law:

$$
\epsilon_{z z_{E}}=\frac{-P_{o} A}{E A_{e f f}}
$$


where,

$$
A_{e f f}=\pi\left(b^{2}-a^{2}+r_{f}^{2}\right)
$$

Here, $\mathrm{A}$ is the outer cross-sectional area at the end cap of the sleeve $\left(\pi b^{2}\right)$ and $A_{\text {eff }}$ represents the effective area which the axial force is being transferred to. The linear combination of Eq. 2.5 and Eq. 2.6 yields an approximate expression for the axial strain induced in the core of the fiber due to external pressure. As the deformation of the structure was not accounted for, we can expect this approximation to differ from the finite element result. However, the expression is useful for discovering the parameters which directly effect the axial strain. The final expression is:

$$
\epsilon_{\text {core }}=\frac{-P_{o} A}{E\left(A_{e f f}\right)}-\frac{\nu}{E}\left(\sigma_{r r}+\sigma_{\theta \theta}\right)
$$

\subsubsection{ANSYS Simulation, External Cavity Design}

An axisymmetric finite element model was created in ANSYS workbench 10 to compare with the analytical result. In most commercial finite element packages today, the simulation begins with creation of the geometry via the preprocessor. Subsequently, the geometry is meshed and the boundary/loading conditions are applied. The type of analysis desired should then be defined. Examples of typical structural analysis include, buckling, modal, static, or nonlinear analysis. 
For the current analysis, an axisymmetric half model of the fiber and surrounding housing was made in ANSYS workbench 10, defining the Y-axis as the axisymmetric axis. The model was meshed using Plane183 elements. The Plane183 element type is a 2-D element with quadratic displacement behavior that can be used for plane stress, plain strain, or axisymmetric simulations. It has 8-nodes with two degrees of freedom at each node; translation in the $\mathrm{x}$ and $\mathrm{y}$ directions. The element includes capabilities such as plasticity, hyperelasticity, creep, stress stiffening, large deflection, and large strain [28].

Figure 2.3 illustrates the model's loading and boundary conditions. It can be noticed that only a static load of 1atm is applied externally along with symmetric boundary conditions, meaning that out-of-plane translations and in-plane rotations are set to zero. It is apparent that a linear static analysis was defined. It is linear because non-linearities are not present, i.e. large deformation, plasticity, creep, stress stiffening, contact (gap) elements, or hyperelastic materials exist, and static because there are no time-varying loads present on the structure. A relatively refined mesh was created and the model was solved. The axial strain through the fiber, as calculated in ANSYS is shown in Fig. 2.4. Table 2.1 gives a detailed description of the geometry and the solution information. Results shown in Fig. 2.4 shows that the outer cavity structure is successful in enhancing the axial strain. The strain on the 


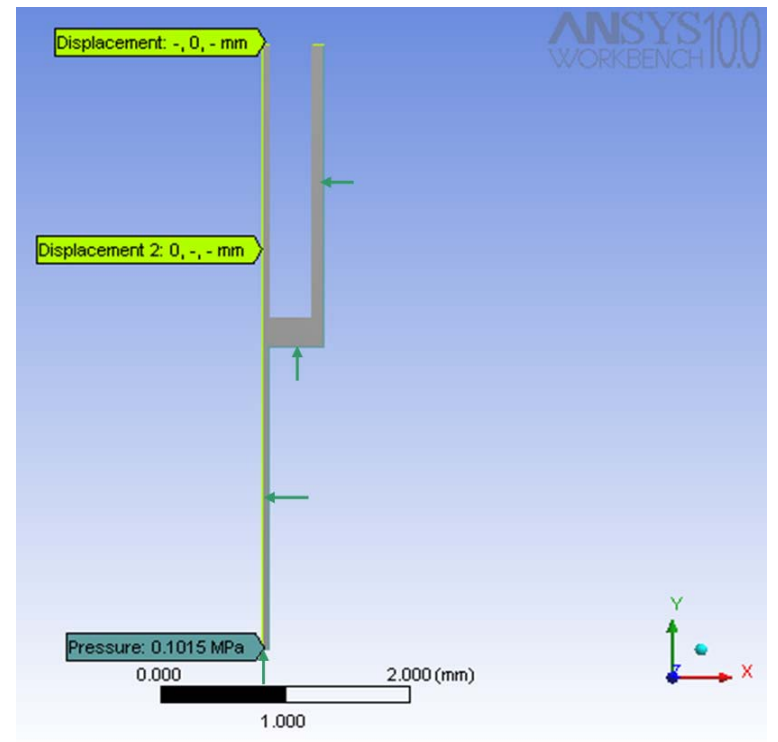

Figure 2.3: Axisymmetric Loading and Boundary Conditions for Fused Capillary Tube Design

fiber itself, due to external pressure of $1 \mathrm{~atm}$, is nominally $-0.8 \mu \epsilon$. The axial strain in the fiber reaches a constant value within the cavity with a magnitude 3.56 times larger than the bare fiber alone.

The cavity design is not a novel idea, but was a good start in the right direction. US patents of similar designs already exist with the same objective to enhance longitudinal strain within a fiber optic cable [29, 30, 31]. 


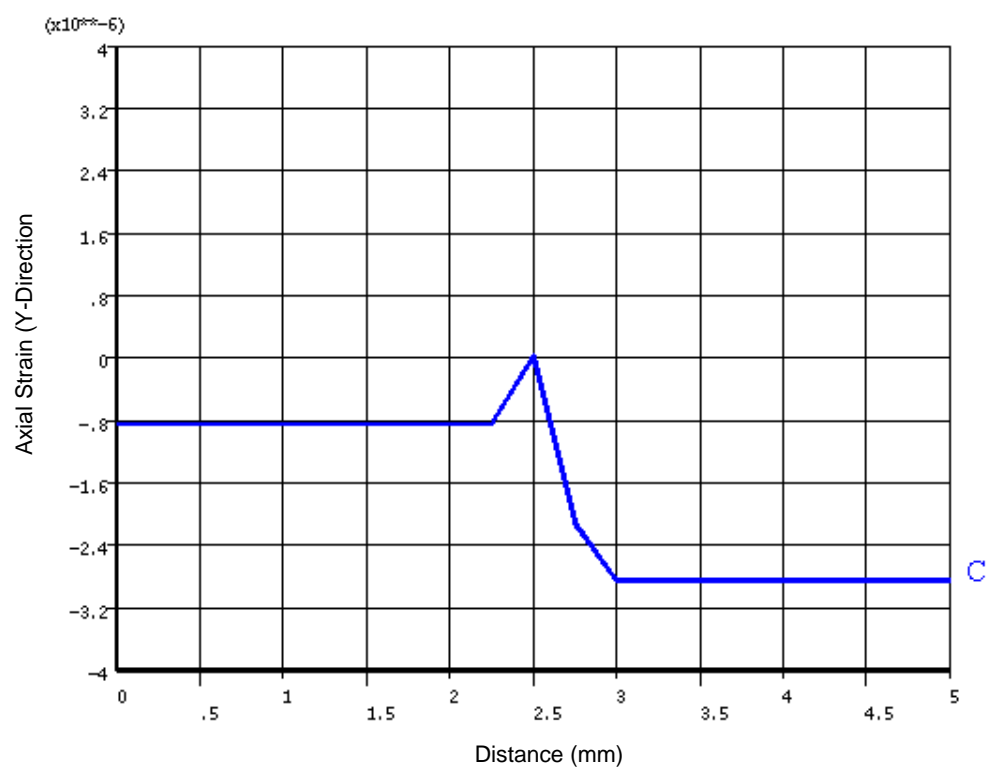

Figure 2.4: Axial Strain Through Fiber Core From ANSYS Simulation Results, starting from $\mathrm{y}=0 \mathrm{~mm}$ to $\mathrm{y}=5 \mathrm{~mm}$ 
Table 2.1: Summary of Simulation Results.*The axial strain results tabulated below refer to a point in the fiber, which is within the surrounding cavity. A comparison is made between the previously derived mathematical expression Eq. 2.8 and the ANSYS simulation results.

\begin{tabular}{|c|l|}
\hline Parameter & Value \\
\hline Tube Outer Diameter (D) & $1 \mathrm{~mm}$. \\
\hline Tube Thickness (t) & $0.1 \mathrm{~mm}$. \\
\hline End Cap Thickness (T) & $0.25 \mathrm{~mm}$. \\
\hline Fiber Diameter & $0.125 \mathrm{~mm}$. \\
\hline Element Type & Plane183 \\
\hline Number Elements used & 2636 \\
\hline Number Nodes used & 7849 \\
\hline Axial Strain FEM & $-2.85 \mathrm{e}-6$ \\
\hline Axial Strain Analytic* & $-2.17 \mathrm{e}-6$ \\
\hline Young's Modulus Glass & $72.4 \mathrm{GPa}$ \\
\hline Poisson Ratio Glass & 0.2 \\
\hline
\end{tabular}

\subsection{The Bellows Approach}

In considering Eq. 2.8, we are limited in what we can do to obtain higher strain sensitivity. It can be noticed that the right hand term yields a tensile strain, that is it is being subtracted from the total compressive strain. Thus, to maximize the compressive strain through the core, one must either reduce Poisson's ratio (optimally to zero), or provide an increase in the Young's modulus of the cavity material. However, increasing the Young's modulus decreases the compressive strain term. Thus we are left with maximizing the first term. It appears that the best way to increase the sensitivity is to increase the diameter of the external cavity. However, due to 
our problem restraints, the diameter of the sensor itself is only allowed a maximum value of $3.175 \mathrm{~mm}$, giving potentially only $-8.55 \mu \epsilon$ for $1 \mathrm{~atm}$ of pressure.

In light of this, a new design approach was considered to further increase the axial strain in the fiber. In this approach, an application of a bellows at the ends of the capillary tube was considered. The choice of a bellows came from the earlier stated design technique of reducing Poisson's effect while decreasing the stiffness. A bellows essentially is a spring with small stiffness that will have the effect of absorbing the tensile strain due to Poisson's effect while allowing more compressive deflection due to the end pressure. Preliminary ANSYS simulations provided exciting results. The modified design yielded 7.6 times more strain at the same pressure difference. Because of this result, plans to create an approximate ten times scaled prototype were made. We felt that an easy to handle prototype would aid in showing proof of the concept while creating a more efficient fabrication procedure. Figure 2.5 illustrates the final bellows based design. All of the commercially available components along with dimensions and the detailed assembly procedure, can be found in appendix A.

\subsubsection{ANSYS Simulation, Bellows Design}

It is apparent from Fig. 2.5 axisymmetric symmetry and half symmetry can be

used. Figure 2.6 depicts the geometry and boundary conditions for the ANSYS 


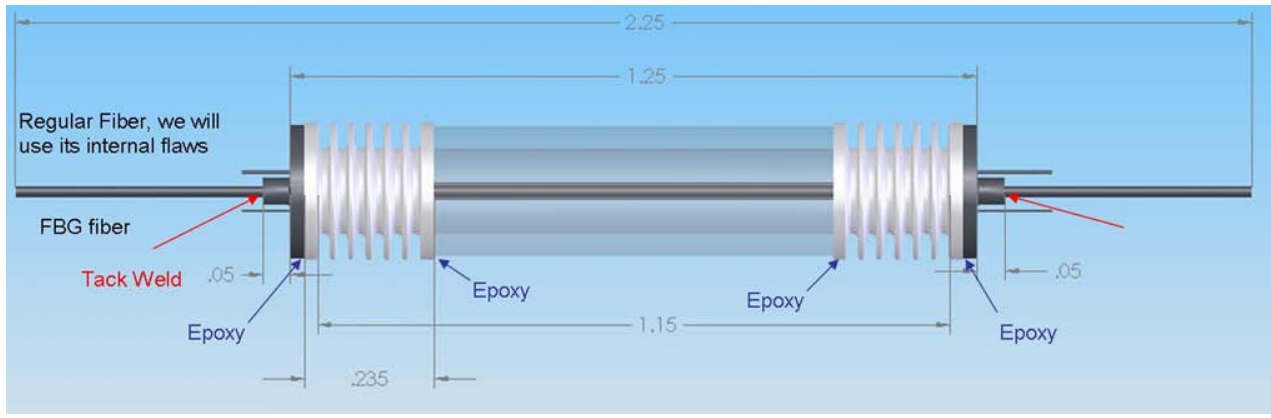

Figure 2.5: The Final Sensor Design with Bellows (Dimensions are in inches, 1 inch $=2.54 \mathrm{~cm})$

Table 2.2: Material Properties, Bellows Model

\begin{tabular}{|c|c|c|c|}
\hline Material & Young's Modulus $(\mathrm{GPa})$ & Poisson's Ratio & Density $\left(\frac{\mathrm{kg}}{\mathrm{m}^{3}}\right)$ \\
\hline Pyrex Glass & 72.4 & 0.2 & 2570 \\
\hline Nickel & 207 & 0.31 & 8880 \\
\hline Stainless Steel & 193 & 0.25 & 2699 \\
\hline
\end{tabular}

environment. The model was meshed using 7,018 Plane2 elements. The Plane2 element is a six-node triangular element with quadratic displacement behavior, well suited for irregular meshes. Each node has two degrees of freedom; displacement in the $\mathrm{x}$ and $\mathrm{y}$ directions. Plasticity, creep, swelling, stress stiffening, large deflection, and large strain effects can be included. The material properties used in the model are shown in Table 2.2. The axial strain results through the core of the fiber from the simulation is shown in Fig. 2.7. 


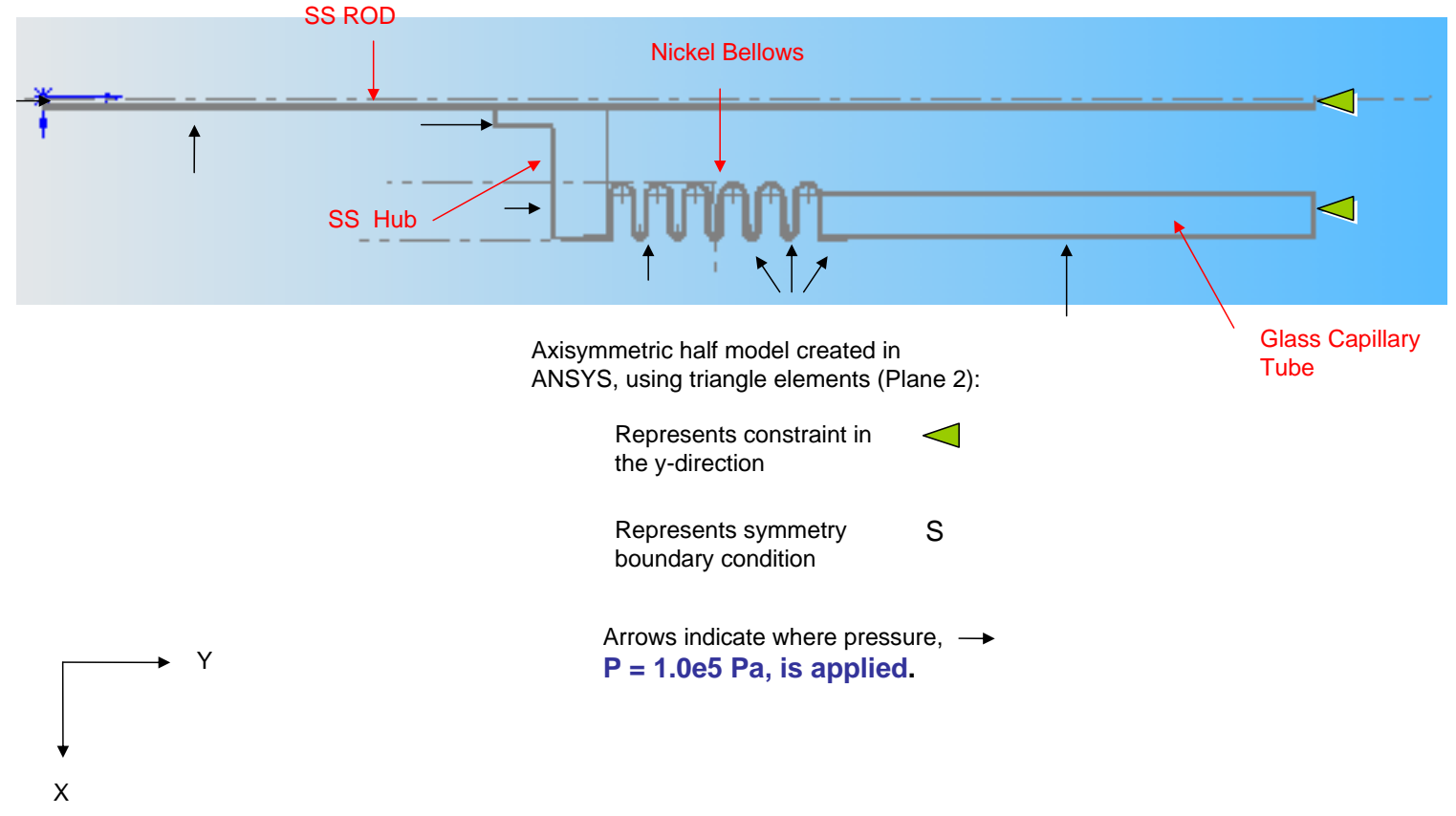

Figure 2.6: ANSYS Geometry and Loading for Bellows Design 


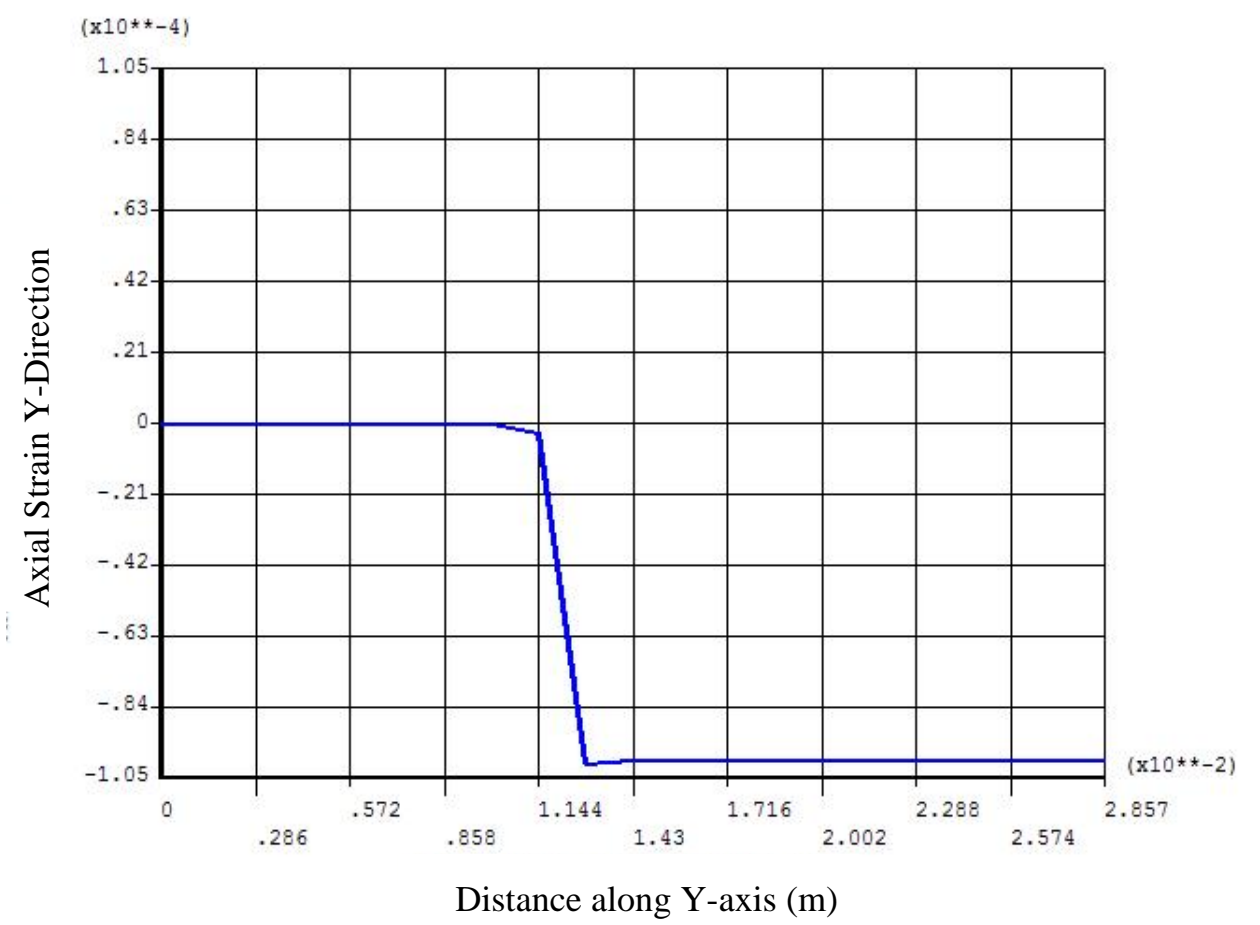

Figure 2.7: Axial Strain Simulation Results Through Core of Fiber, Bellows Design 


\subsubsection{Mathematical Model, Bellows Design}

The constant value of $-100 \mu \epsilon$ found in Fig. 2.7 was validated using a simple, one dimensional, spring model. In this model, the structural components are represented as a linear springs with stiffness $\mathrm{K}$ 's, see Fig. 2.8. Here $K_{B}, K_{G}$, and $K_{S}$ represent

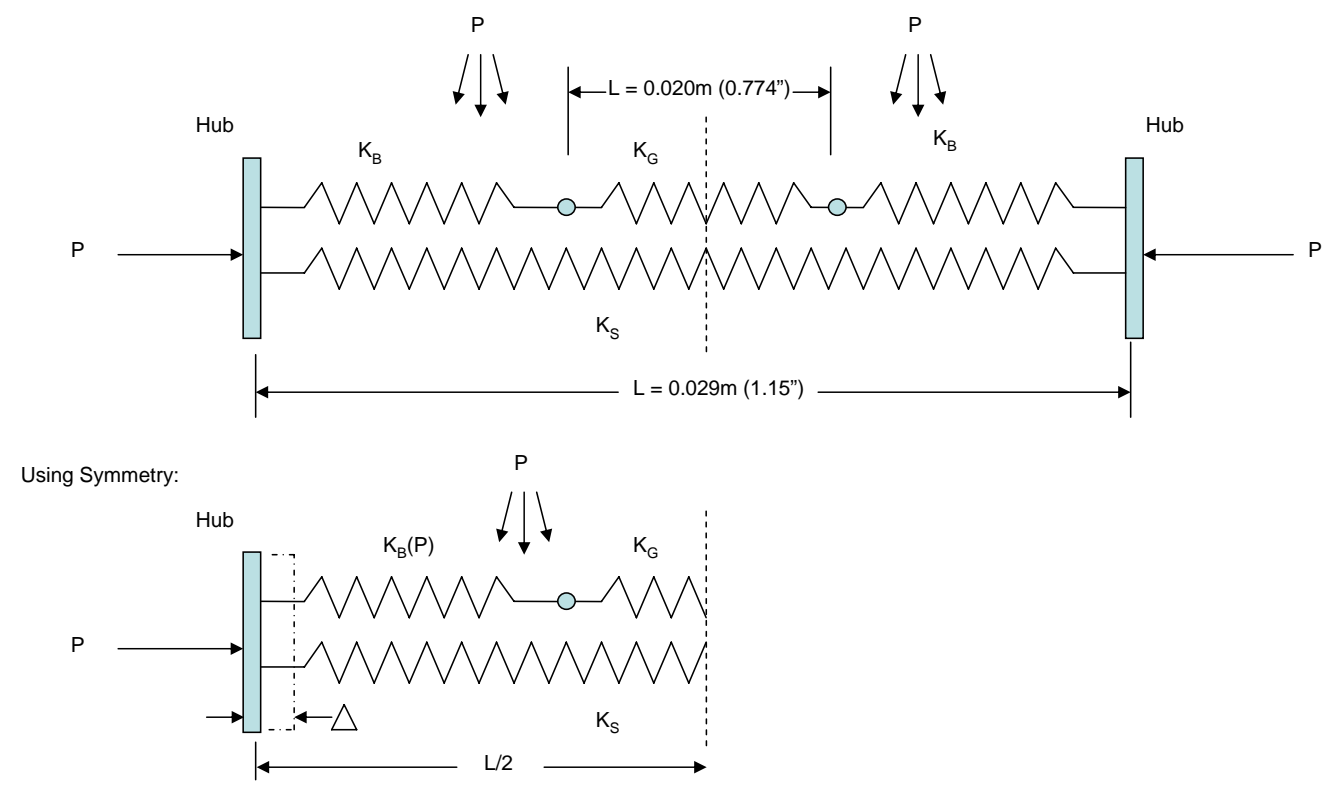

Figure 2.8: Bellows Spring Model

the axial stiffness of the bellows, glass tube and the stainless steel rod respectively.

The bellows stiffness was found to be, $K_{B}=16313 \frac{N}{m}$, using ANSYS. Here, an ax- 
isymmetric bellows model was created using dimensions and material as specified by the manufacturer. A unit load was applied to the tip while the other end was constrained, the resulting displacement was noted. $K_{G}$ and $K_{S}$ are given from the axial bar relation, $P=\frac{E A}{L} u$, that is, $K=\frac{E A}{L}$. Where $\mathrm{E}$ is the Young's modulus of the material, $\mathrm{A}$ is the area of cross-section and $\mathrm{L}$ is the length. Using this relation the glass and rod stiffnesses were calculated to be, $K_{G}=1.14 E 08 \frac{N}{m}$ and $K_{S}=1.38 E 06 \frac{N}{m}$. It is apparent that $K_{B}<<K_{G}$. Thus the equivalent stiffness of the upper springs in series, becomes just, $K_{e q} \cong K_{B}$ and the problem simplifies further, Fig. 2.9. Now

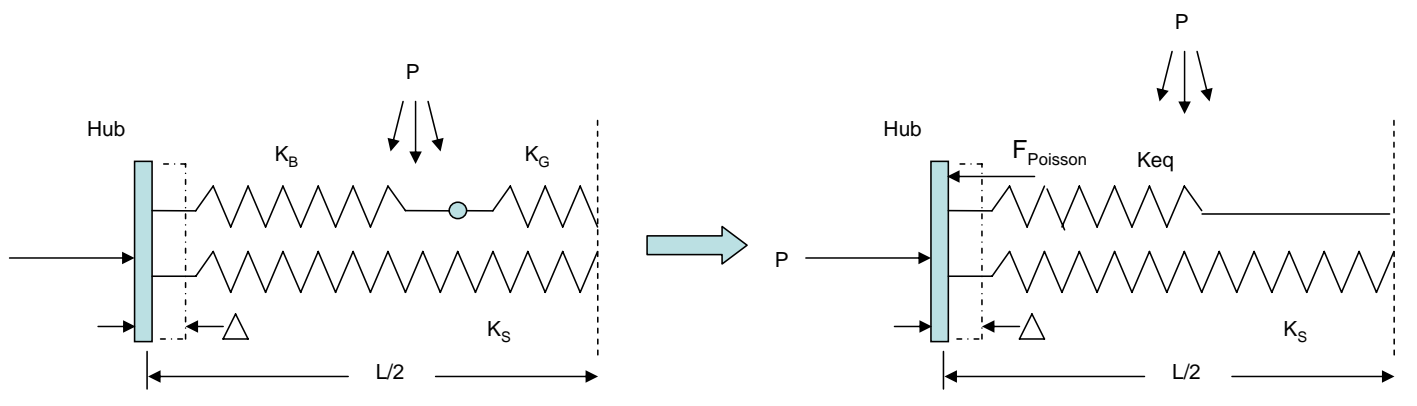

Figure 2.9: Reduced Bellows Spring Model

the displacement of the hub and therefore the stainless steel rod can be determined from the following relation:

$$
\Delta=\frac{F_{h u b}-F_{\text {Poisson }}}{K_{B}+K_{S}}
$$


Here, $F_{h u b}$ is the force acting on the hub from the external pressure, $F_{h u b}=P A_{h}=$ $3.20 N$, where $A_{h}$ is the cross-sectional area of the hub and $\mathrm{P}$ is the external pressure applied. $F_{\text {Poisson }}$ is the force generated in the glass from Poisson's effect plus the additional net force due to the extension of the bellows under pressure. Predicting the behavior of a bellows is not the aim of this project, which is why ANSYS has been used to define its parameters.

$$
F_{\text {Poisson }}=P A_{\text {Bell }}+\frac{\epsilon_{G} L_{G} K_{B}}{2}
$$

Here, $\epsilon_{G}$ is the axial strain induced by Poisson effect in the glass tube, this value can be found through use of Airy's stress function. The axial strain value found in this case is very small, $\epsilon_{G}=1.0 E-06 \mu \epsilon$, and thus this term is neglected. The $A_{\text {Bell }}$ is the effective planar area in which the pressure acts. Because of the bellows irregular shape, this value was found through trial and error using ANSYS to be $1.01 E-05 \mathrm{~m}^{2}$. Now with the known displacement of the stainless steel rod, the compressive axial strain can be calculated,

$$
\epsilon_{s}=\frac{2 \Delta}{L}=103 \mu \epsilon
$$


Notice, that this value agrees closely with the ANSYS simulation results, shown in Fig. 2.7.

\subsubsection{Fabrication and Preliminary Testing Results, Bellows}

\section{Design}

A detailed assembly procedure of the sensor prototype can be found in Appendix A. The final product is shown in Fig. 2.10. The sensor was tested in an enclosed

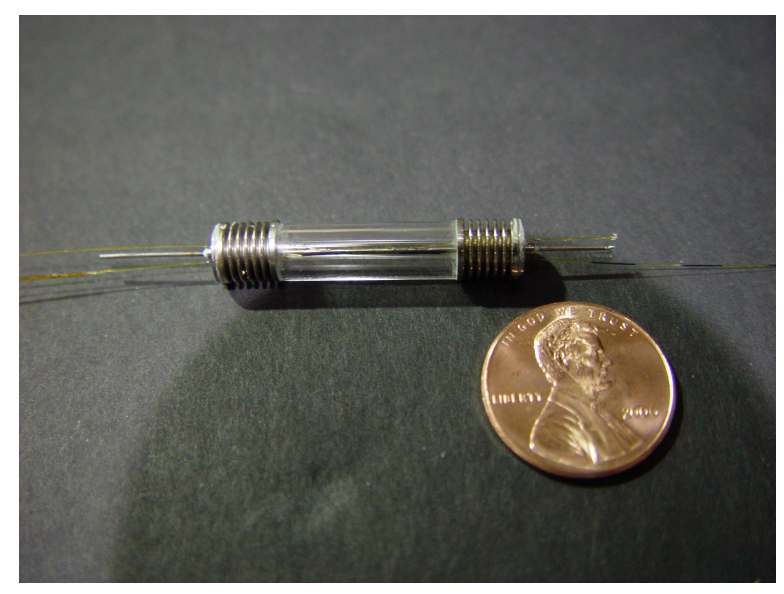

Figure 2.10: The Prototype After Fabrication

chamber, the pressure inside the chamber began at 0 psi and was stepped up in 5 psi increments, every 5 minutes, up to 55 psi and back down to 5 psi. The strain induced in a fiber bonded on the stainless steel rod was monitored with Luna's distributed sensing system (DSS). A complete summary of the test setup can be found in Appendix B. A summary of the testing results is presented in the following 
graph: The overall general behavior of the sensor was observed to be as expected.

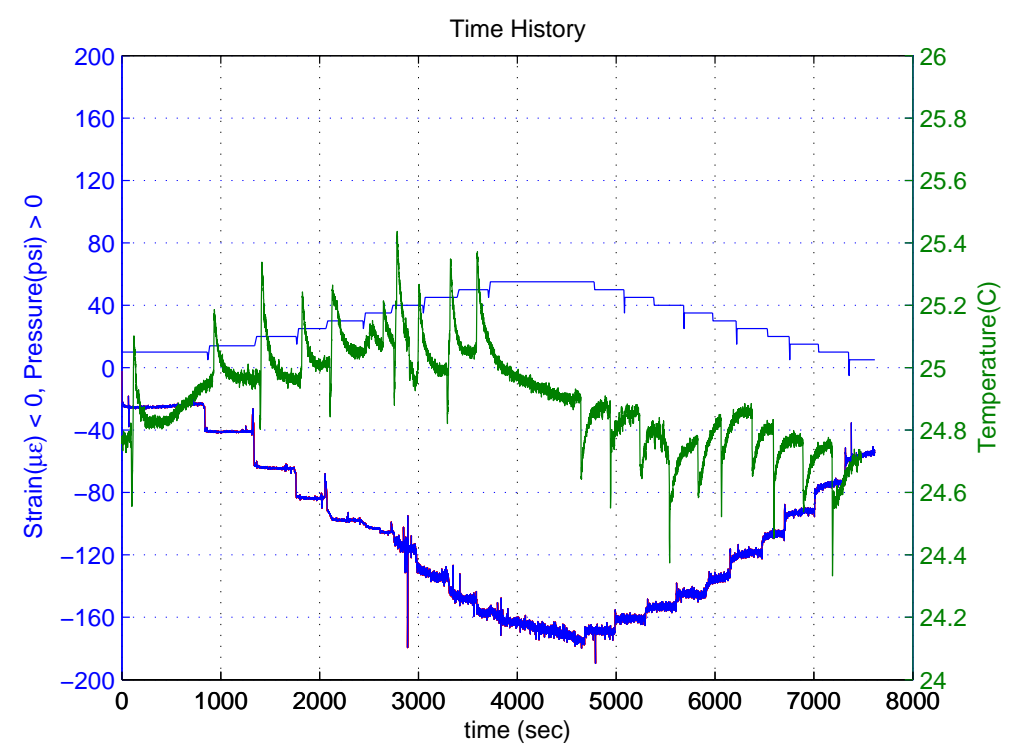

Figure 2.11: Preliminary Testing Results; Pressure(blue, $y>0$ ), Strain(blue, $y<0$ ), and Temperature(green) as a function of time

That is, as the pressure was cycled up to 55 psi and back down, the compressive axial strain is steadily increasing then decreasing. However, the results from the test did not correspond exactly to the numerical predictions found doing an analysis using ANSYS. The numerical results using the ANSYS model described above depict a linear variation of strain with an increase of the pressure difference. A plot comparing the numerical and experimental results is shown in Fig. 2.12. Hysteresis can be observed from Fig. 2.12. That is, the system does not respond to the same forces in the same manor on the path while cycling the pressure back down to 5 psi. Also, the compressive axial strain from the experiment is considerably lower than the 


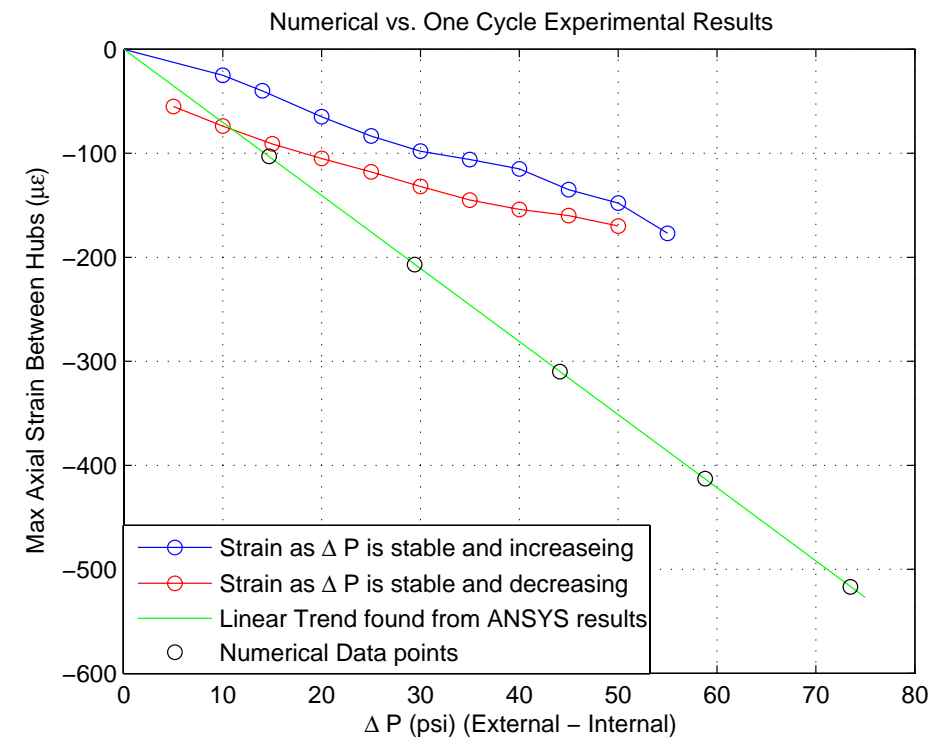

Figure 2.12: Numerical vs. Experimental Strain Results; One Cycle Numerical Comparison with Actual Test Results, Here the Strain Points from the Experiment were taken when the Temperature within the Test Chamber had Stabilized back to room temperature 
expected numerical simulation results. These discrepancies have been attributed to the combination of poor bonding between the fiber and stainless steel rod and more notably, the effect of the end reflection wash out, due to one of the fibers being cut to close to the grating. An indication of bond failure can be seen in the axial strain data of Fig. 2.11, it can be noticed in the time interval from [0-2000s], that the strain data is stepping down and the data is clear, shortly thereafter, between [2000-8000s], the strain signal becomes very fuzzy. Most likely indicating that the fiber has become loose. Another indication of bond failure stems from the fact that the strain data is not symmetric as would be expected.

The low values of strain detected throughout the testing has been attributed to signal wash out. This can be seen on the right side of Fig. 2.10. It can be noticed that one fiber has been cut too short, leaving an interface to the ambient air very near the grating. Any such interface has a tendency to cause large reflections of light. Some of this end reflection may have washed out a portion of our strain signal.

\subsubsection{Conclusions, Bellows Design}

Initially the bellows design showed promising results. The major shortcomings of the design, however, are the multiple adhesive bondings needed between many small

parts, making it very difficult to be mass produced on a $\frac{1}{8}$ inch scale. Because of this 
disadvantage, a new design approach was introduced, one whose key feature is easy fabrication. 


\section{Chapter 3}

\section{Diaphragm Design}

\subsection{Motivation}

Follwing the bellows concept, we considered several pressure sensor designs from the perspective of performance and cost. Initially, an alternative design, including a recessed elliptical diaphragm (Fig. 3.1), was proposed. This design was motivated by previous work done by Luna Energy. Diaphragm sensors were considered easier to fabricate than the original bellows design by reducing complex bonding of different materials. To further simplify machining, a diaphragm sensor created from a notched round tube was proposed, (Fig. 3.2) but it's sensitivity was found to be inadequate according to ANSYS simulations (Fig. 3.3). In the 3D model, Solid95 
elements were used to mesh a quarter model of a single notched tube, refer to Fig. 3.2. The boundary conditions were such that, the end away from the notch was fixed, and symmetry boundary conditions were imposed on the symmetry faces. Solid95 is a 3-D 20-node element with three degrees of freedom at each node, translation in the $\mathrm{x}-\mathrm{y}-\mathrm{z}$ directions, which tolerates irregular meshes without much loss of accuracy. Notice in Fig. 3.3(a), that the tensile strain is concentrated only in the thinnest region of the diaphragm (shown in red) and there is a small region of compressive strain in the tapered boundaries (shown in darker blue). Figure 3.3(b), shows a plot of the axial strain along the inner wall of the stainless steel tube as a function of the distance from the thinnest region. It can be noticed that the axial strain distribution is not evenly distributed and the maximum tensile strain is only $3.5 \mu \epsilon$, which would be barely detectable using the optical backscatter reflectometer (OBR, Luna Innovations' interferometer device to measure strain). The next approach proved to be an ideal one, as it provides ease in production along with desirable sensitivity at our target operating pressure 2.413 MPa (350 psi).

Finally, a milled diaphragm in a square cross-section tube (Fig. 3.4) provided both simple machining and reasonable theoretical pressure sensitivity. The concept is simple, pressure is detected by a deflection of the diaphragm, which creates tensile strain on an optical fiber bonded to the opposite side of the diaphragm in an internal 


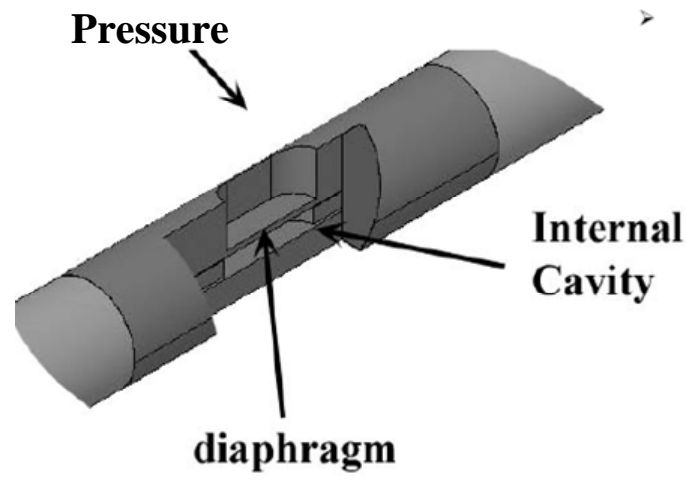

Figure 3.1: Luna Energy Diaphragm Sensor Concept; a differential pressure causes the diaphragm to deflect into an internal cavity, at atmospheric pressure, straining an optical fiber directly underneath.

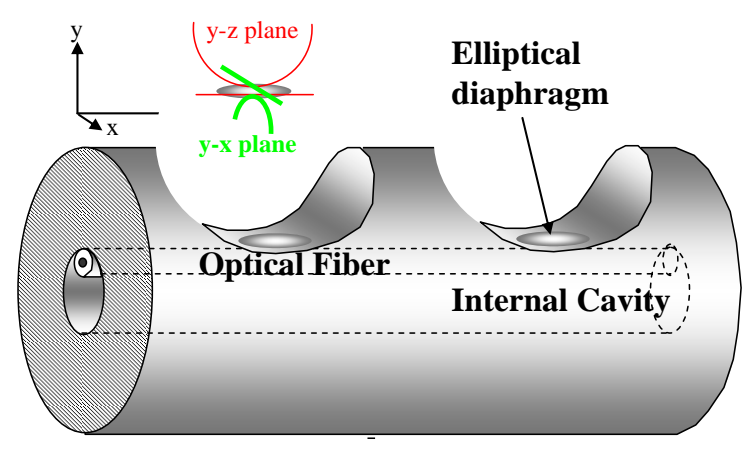

Figure 3.2: Notches Milled into a Circular Tube, Forming an Elliptical Diaphragm 


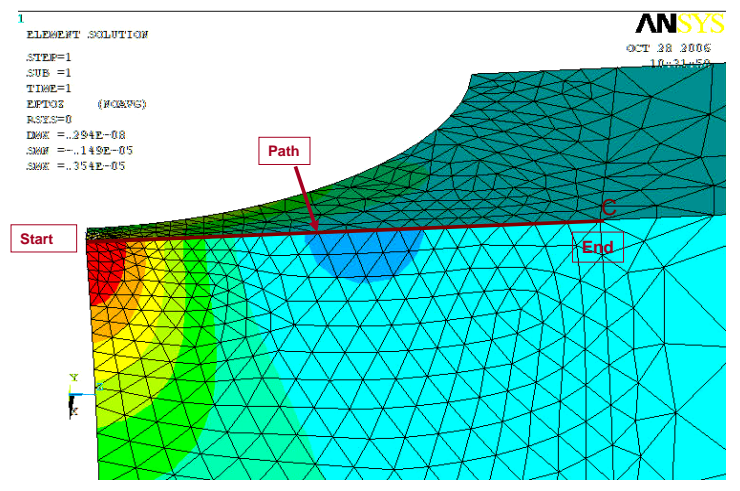

(a) Closeup of mesh and axial strain contours of portion just underneath the diaphragm section

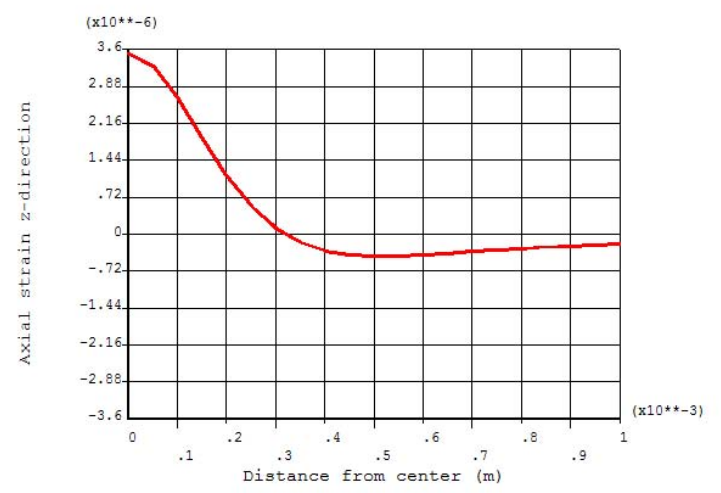

(b) Axial strain vs. Distance along path; Graphically depicts the strain distribution from the ANSYS model

Figure 3.3: ANSYS model of a pressure sensor formed from a $3.175 \mathrm{~mm}(0.125 ")$ diameter stainless steel tube with a $0.7874 \mathrm{~mm}(0.031$ ") wall thickness and a circular notch $\left(\mathrm{r}=0.7112 \mathrm{~mm}\left(0.028^{\prime \prime}\right)\right)$, resulting in a minimum thickness of $0.0762 \mathrm{~mm}$ (0.003"). See Fig. 3.2 for a more conceptual sketch of the sensor. Applied pressure, $2.413 \mathrm{MPa}(350 \mathrm{psi})$ 
cavity at a lower reference pressure. ANSYS simulations of the behavior of the diaphragm itself was done in order to recommend dimensions for a prototype pressure sensor. Since FBGs can detect strain with a noise floor sensitivity of about $2 \mu \epsilon$, the goal was set to measure at least $200 \mu \epsilon$ at target pressure of 350 psi $( \pm 50 \%)$ while keeping the outside diameter within a constraint of $\frac{1}{8}$ inch. Under these conditions, the signal-to-noise ratio would be above 80 .

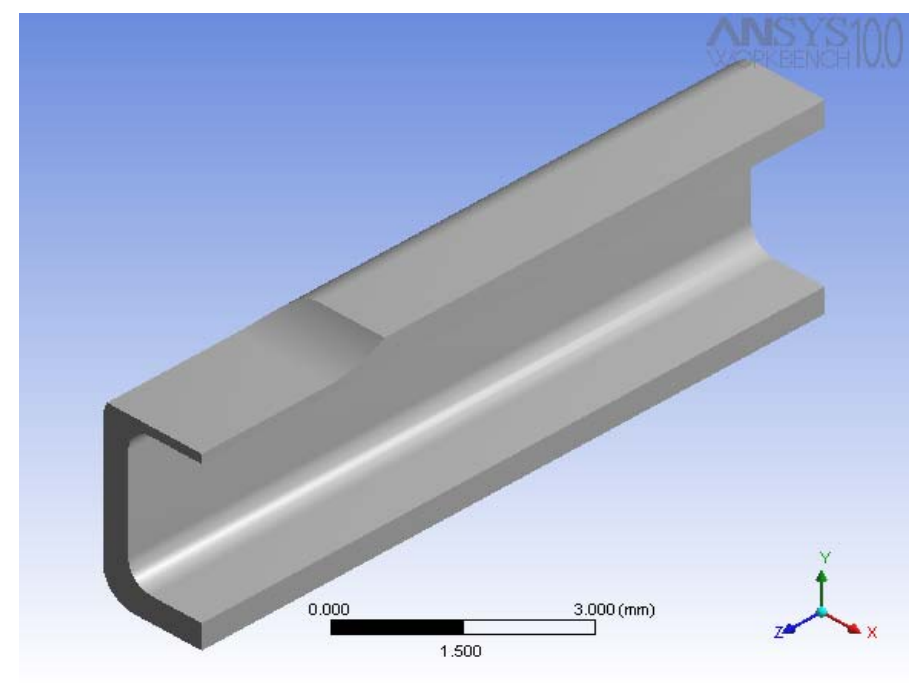

Figure 3.4: Square Tube with Machined Diaphragm; Quarter model of a $3.175 \mathrm{~mm} x$ $3.175 \mathrm{~mm} \times 0.4064 \mathrm{~mm}\left(\frac{1}{8}\right.$ inch $\times \frac{1}{8}$ inch $\times 0.016$ inch $)$ square tube is shown. A milled diaphragm is depicted with thickness, $150 \mu \mathrm{m}$ a flat length of $2 \mathrm{~mm}$. The flat portion of the diaphragm is transitioned with the square tube through a $1.5875 \mathrm{~mm}$ radius 


\subsection{Brief Review of Nonlinear Finite Element Anal- ysis and Shell Elements}

In the following developments a nonlinear finite element simulation using shell elements was performed. This review is intended to aid the reader in understanding the concepts used and why design decisions were made.

\subsubsection{Nonlinearities in Structures}

Nonlinearities occur often in real world situations and are apparent in many engineering problems. Under certain simplifying assumptions, these problems can be described as linear, which is typically done in industry. Nonlinear structural behavior can be grouped into three categories, changing status, geometrical nonlinearities, and material nonlinearities [28]. Many structural problems exhibit nonlinear behavior that is displacement or load dependent, for instance, a roller support is sometimes in contact or not in contact.

A geometric nonlinearity occurs when a structure experiences large deformation, because its resulting geometric configuration corresponds to a nonlinearity. In structural mechanics, the change in the geometric shape, i.e. the curvature of a plate, can no longer be treated small when large strains or large deflections are present, 
as the strain field becomes dependent on nonlinear effects that cannot be ignored. Geometric nonlinear structures are visualized in their load vs. displacement curve, Fig. 3.5(a)

Material nonlinearities occur when the stress-strain relationship becomes nonlinear. Many factors can influence the stress-strain relationship, for instance, load history (elastic plastic regimes), amount of time the load is applied (creep), and environmental changing conditions (temperature) [28]. Figure 3.5(b) shows stress as a nonlinear function of strain. Solving nonlinear problems with the finite element method is a broad, detailed subject, and will not be given here in detail. However, J.N. Reddy gives an excellent introduction to nonlinear finite element analysis in [32].
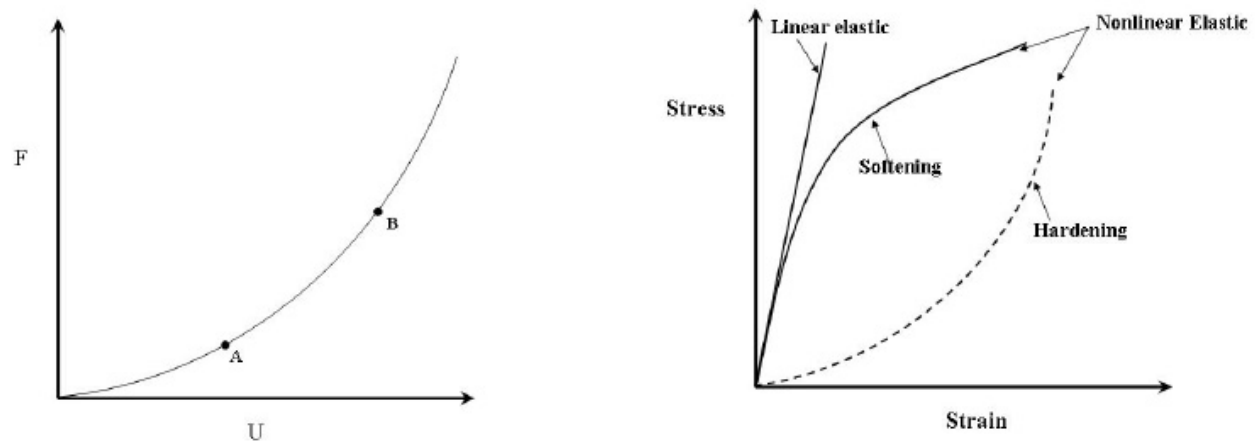

(a) Geometric nonlinear structure; Load (b) Material nonlinear structure; Stress vs. Strain vs. Displacement

Figure 3.5: Structural nonlinearites 


\subsubsection{Shell Elements in ANSYS}

Shell structures are characterized as a three dimensional body with its thickness small as compared to the other two dimensions. Shell structures are very common in virtually every discipline. Their light weight and high strength makes them ideal for aircraft, spacecraft, and automobile body components. Small thin plates are also very important to the micro pressure sensing technologies, MEMS depend heavily on the deflection of very small diameter circular diaphragms. ANSYS provides a library of highly accurate shell elements, [28], in the following elliptical diaphragm analysis, the ANSYS element Shell181 was used.

Shell181, is well-suited for linear, large rotation, and/or large strain nonlinear applications, and also for analyzing thin to moderately thick shell structures. It is a 4-node element with six degrees of freedom at each node; translations in the $\mathrm{x}, \mathrm{y}$, and $\mathrm{z}$ directions, and rotations about the $\mathrm{x}, \mathrm{y}$, and z-axes, while having the capability to toggle between both membrane (in-plane stresses) and bending element formulation or membrane only formulation. If membrane only is chosen, the element has translational degrees of freedom only. In our analysis the membrane and bending stiffness option was used. Some other special features include, plasticity, large deflection, creep, stress stiffening, large strain, and initial stress input.

The objective in the following analysis was to determine the optimal diaphragm 
configuration, that is, to find the dimensions of the diaphragm which would yield our target strain, $200 \mu \epsilon$ at $2.413 \mathrm{MPa}$ (350 psi), evenly distributed over at least $4 \mathrm{~mm}$ (the minimal length in which a clear strain signal could be obtained using Luna's interrogation system). A parameter investigation was done by writing an input file in ANSYS, designed to loop through the various geometrical configurations of a diaphragm, each with the same uniform pressure load and clamped boundary conditions, and outputting the transverse deflection at the center along with the axial strain distribution through the semimajor axis. The capabilities of Shell181 fit this analysis nicely, the various geometries given in the input file may yield geometric nonlinearites. Also the option to add an initial stress state is appealing.

In order to determine whether our finite element results were valid, we compared them with results obtained from the classical theory of plates.

\subsection{Deflection of an Elliptical Plate}

A plate is a flat body, symmetric with respect to a plane dividing its height and whose thickness is considered small when compared to its other dimensions. Many structural elements can be treated as plates, especially in the development of diaphragm based pressure sensor transducers. Having an analytical solution available is highly desirable in the development of small diaphragm based pressure sensors. 
Eaton, et al. developed a new analytical solution for the deflection of clamped circular plates applicable to MEMS based systems [33]. For convenience, the diaphragm shape to be used in our parameter optimization was chosen to be the same as that in the Luna design, Fig. 3.1. Because it is an elliptical like shape, in the following paragraphs, solution to the clamped elliptical plate subjected to uniform pressure is sought [34]. First, however, an introduction to classical plate theory is presented.

\subsubsection{Small-Deflection Theory}

Consider the bending of a plate due to a load intensity, q, distributed over the surface. The governing equation for the plate can be derived from the equilibrium equations for a plate element with uniformly distributed bending moments over the plate's edges $[35,36]$. The thickness of the plate is denoted by $h$, and is positive in the positive z-direction. This derivation comes directly from the classical theory of the bending of plates. In this derivation the following assumptions, including Kirchoff hypothesis' are used [36],

1. The material of the plate is elastic, homogeneous, continuous, and isotropic

2. The bending deflections are small compared to the thickness of the plate and do not alter the plate's geometry. Thus, the slope of the deflected surface is small and its square is a negligible quantity. 
3. The deformations are such that the straight lines normal to the midplane before bending remain straight and normal to the middle surface after bending. Therefore shear strains, $\gamma_{x z}$ and $\gamma_{y z}$ are negligible.

4. The transverse normals do not experience elongation, $\epsilon_{z z}=0$

5. The deflections of the plate are due to the displacements of points of the mid surface in the direction normal to the initial plane.

The following equation results after application of equilibrium of forces and moments on the plate element:

$$
\frac{\partial^{2} M_{x}}{\partial x^{2}}-2 \frac{\partial^{2} M_{x y}}{\partial x \partial y}+\frac{\partial^{2} M_{y}}{\partial y^{2}}=-q
$$

We want to express the obtained equation in terms of the plate's deflection, $\mathrm{w}(\mathrm{x}, \mathrm{y})$. To do so, the moments must be evaluated using:

$$
\begin{aligned}
M_{x}=\int_{-h / 2}^{h / 2} \sigma_{x x} z d z & M_{y}=\int_{-h / 2}^{h / 2} \sigma_{y y} z d y \\
M_{x y}=\int_{-h / 2}^{h / 2} \tau_{x y} z d z & M_{y x}=\int_{-h / 2}^{h / 2} \tau_{y x} z d z
\end{aligned}
$$


The kinematics resulting from pure bending show that the normal and shear strain expressions are:

$$
\begin{array}{r}
\epsilon_{x x}=\frac{z}{\rho_{x}} \quad \epsilon_{y y}=\frac{z}{\rho_{y}} \\
\gamma_{x y}=-2 z \frac{\partial^{2} w}{\partial x \partial y}
\end{array}
$$

where for small deflections the principal curvatures are,

$$
\frac{1}{\rho_{x}}=-\frac{\partial^{2} w}{\partial x^{2}}, \quad \frac{1}{\rho_{y}}=-\frac{\partial^{2} w}{\partial y^{2}}
$$

Using Hooke's law and the above strain relationships, Eq. 3.1 can be reformulated into the well known expression:

$$
\nabla^{4} w=\frac{q}{D}
$$

where, $D=E h^{3} / 12\left(1-\nu^{2}\right)$ is known as the flexural rigidity. There are two common methods typically used to solve Eq. 3.7. The Ritz method is one option. In this method the deflection is represented as a summation of constants (Ritz coefficients) and appropriately chosen functions, Eq. C.6 (Appendix C). For a linear elastic material, the total potential energy $(\Pi=U+V)$ for the plate can be obtained from 
its strain energy (U) and the potential of the external loading (V). Subsequently an assumed approximate deflection is substituted into the total potential energy and minimized to obtain the Ritz coefficients, leading to an approximate solution for the deflection. Using Bubnov-Galerkin method one can arrive at the same expression for deflection [36]. This method is a weak form approach, that is, the appropriate deflection profile (in terms of trial functions) are substituted into the equilibrium equation, Eq. 3.1, multiplied by a weight function, which is just the displacement (Galerkin), and integrated over the plate's area, the resulting expression is then set equal to zero.

The following one term approximation yields the exact solution for an elliptical plate subject to small deformations and clamped boundary conditions, [27].

$$
\begin{array}{r}
w=a_{1} \Phi_{1} \\
\Phi_{1}=\left[\left(\frac{x}{a}\right)^{2}+\left(\frac{y}{b}\right)^{2}-1\right]^{2}
\end{array}
$$

Notice that $\Phi$, resembles the equation of an ellipse, where $a$ is the semimajor axis and $b$ is the semiminor axis. Using the method of minimum total potential energy, the one-term solution is found to be [27]:

$$
w=\frac{q}{D\left(24 / a^{4}+24 / b^{4}+16 / a^{2} b^{2}\right)}\left[\left(\frac{x}{a}\right)^{2}+\left(\frac{y}{b}\right)^{2}-1\right]^{2}
$$




\subsubsection{Large Deformation Theory}

In the small deflection theory development we neglected forces in the plane of the plate, also known as membrane forces. Timoshenko [35], suggests that small deflection theory becomes inaccurate when the transverse deflections are larger than 0.5h. To obtain more accurate solutions, we can reformulate the governing equations to include membrane effects. Consider a differential plate element in the xy plane, with normal and shear forces acting, $T_{x}, T_{y}, T_{x y}$. The in-plane equilibrium equations become [36]:

$$
\begin{aligned}
& \frac{\partial T_{x}}{\partial x}+\frac{\partial T_{x y}}{\partial y}=0 \\
& \frac{\partial T_{x y}}{\partial x}+\frac{\partial T_{y}}{\partial y}=0
\end{aligned}
$$

To add the effects of membrane forces to the transverse load $q$, in Eq. 3.7, we must calculate the projection of the in-plane forces in the z-direction:

$$
T_{x} \frac{\partial^{2} w}{\partial x^{2}}+T_{y} \frac{\partial^{2} w}{\partial y^{2}}+2 T_{x y} \frac{\partial^{2} w}{\partial x \partial y}
$$

The modified Eq. 3.7, becomes:

$$
\nabla^{4} w=\frac{1}{D}\left(q+T_{x} \frac{\partial^{2} w}{\partial x^{2}}+T_{y} \frac{\partial^{2} w}{\partial y^{2}}+2 T_{x y} \frac{\partial^{2} w}{\partial x \partial y}\right)
$$


The task of relating the membrane forces to the deflection still exists. It can be shown that the strain in the midplane takes on additional terms dependent of the deflection.

$$
\begin{aligned}
\epsilon_{x}^{\circ} & =\frac{\partial u}{\partial x}+\frac{1}{2}\left(\frac{\partial w}{\partial x}\right)^{2} \\
\epsilon_{y}^{\circ} & =\frac{\partial v}{\partial y}+\frac{1}{2}\left(\frac{\partial w}{\partial y}\right)^{2} \\
\epsilon_{x y}^{\circ} & =\frac{\partial u}{\partial x}+\frac{\partial v}{\partial y}+\frac{1}{2} \frac{\partial w}{\partial x} \frac{\partial w}{\partial y}
\end{aligned}
$$

resulting in the following compatibility condition

$$
\frac{\partial^{2} \epsilon_{x}}{\partial y^{2}}+\frac{\partial^{2} \epsilon_{y}}{\partial x^{2}}=\left(\frac{\partial^{2} w}{\partial x \partial y}\right)^{2}-\frac{\partial^{2} w}{\partial x^{2}} \frac{\partial^{2} w}{\partial y^{2}}
$$

Combining Eq. 3.17, Eq. 3.18, and Hooke's Law, results in the following differential equation:

$$
\frac{\partial^{2} T_{x}}{\partial y^{2}}-\nu \frac{\partial^{2} T_{y}}{\partial y^{2}}+\frac{\partial^{2} T_{y}}{\partial x^{2}}-\nu \frac{\partial^{2} T_{x}}{\partial x^{2}}-2(1+\nu) \frac{\partial^{2} T_{x y}}{\partial x \partial y}=E h\left[\left(\frac{\partial^{2} w}{\partial x \partial y}\right)^{2}-\frac{\partial^{2} w}{\partial x^{2}} \frac{\partial^{2} w}{\partial y^{2}}\right]
$$


Introducing the stress function $\phi$ :

$$
\begin{array}{r}
\sigma_{x}=\frac{T_{x}}{h}=\frac{\partial^{2} \phi}{\partial y^{2}}, \quad \sigma_{y}=\frac{T_{y}}{h}=\frac{\partial^{2} \phi}{\partial x^{2}} \\
\sigma_{x y}=\frac{T_{x y}}{h}=\frac{-\partial^{2} \phi}{\partial x \partial y}
\end{array}
$$

Finally, the governing equations of a plate for large deflections, known as von Kármán's equations are:

$$
\begin{aligned}
& D \nabla^{4} w=h L(w, \phi)+q, \quad \frac{1}{E} \nabla^{4} \phi=\frac{1}{2} L(w, w) \\
& L(w, \phi)=\frac{\partial^{2} \phi}{\partial y^{2}} \frac{\partial^{2} w}{\partial x^{2}}-2 \frac{\partial^{2} \phi}{\partial x \partial y} \frac{\partial^{2} w}{\partial x \partial y}+\frac{\partial^{2} \phi}{\partial x^{2}} \frac{\partial^{2} w}{\partial y^{2}}
\end{aligned}
$$

An approximate solution to the von Kármán equations for an elliptical plate under uniform pressure, was presented by W.A. Nash and I.D. Cooley, [34]. They arrived at their approximate solution by employing the perturbation method based on the smallness of the central deflection of the elliptical plate. This means that they denote the uniform pressure loading, and all three components of displacement as a 
series of ascending powers of the central deflection, $W_{o}$.

$$
\begin{aligned}
q & =\alpha_{1} W_{o}+\alpha_{3} W_{o}^{3}+\ldots \\
w(x, y) & =w_{1}(x, y) W_{o}+w_{3}(x, y) W_{o}^{3}+\ldots \\
u(x, y) & =s_{2}(x, y) W_{o}^{2}+s_{4}(x, y) W_{o}^{4}+\ldots \\
v(x, y) & =t_{2}(x, y) W_{o}^{2}+t_{4}(x, y) W_{o}^{4}+\ldots
\end{aligned}
$$

Here, $\mathrm{q}$ is the uniform pressure, $\mathrm{w}(\mathrm{x}, \mathrm{y})$ is the transverse deflection, $\mathrm{u}(\mathrm{x}, \mathrm{y})$ is the displacement in the $\mathrm{x}$-direction and $\mathrm{v}(\mathrm{x}, \mathrm{y})$ is the displacement in the $\mathrm{y}$-direction. It is necessary that $w_{1}(0,0)=1$ and $w_{j}(0,0)=0, j=1 . . n$. Polynomial approximations which satisfy the boundary conditions are made and substituted into the von Kármán equations. Equating appropriate terms involving $\mathrm{x}$ and $\mathrm{y}$, yields the needed linear equations to be used to solve for the coefficients in the polynomial approximations. Figure 3.6 is a plot showing how their solution deviates from the linear solution. Notice, when a dimensionless load of $\frac{q b^{4}}{E h^{4}}>1$, the straining of the midplane can no longer be neglected and the von Kármán equations must be used to attain an accurate solution. Nash and Cooley [34], compared their results to experimental data they collected. It is worth noting that their solution accurately predicted the experimental results for $0<\frac{q b^{4}}{E h^{4}}<4$, beyond this the error between 


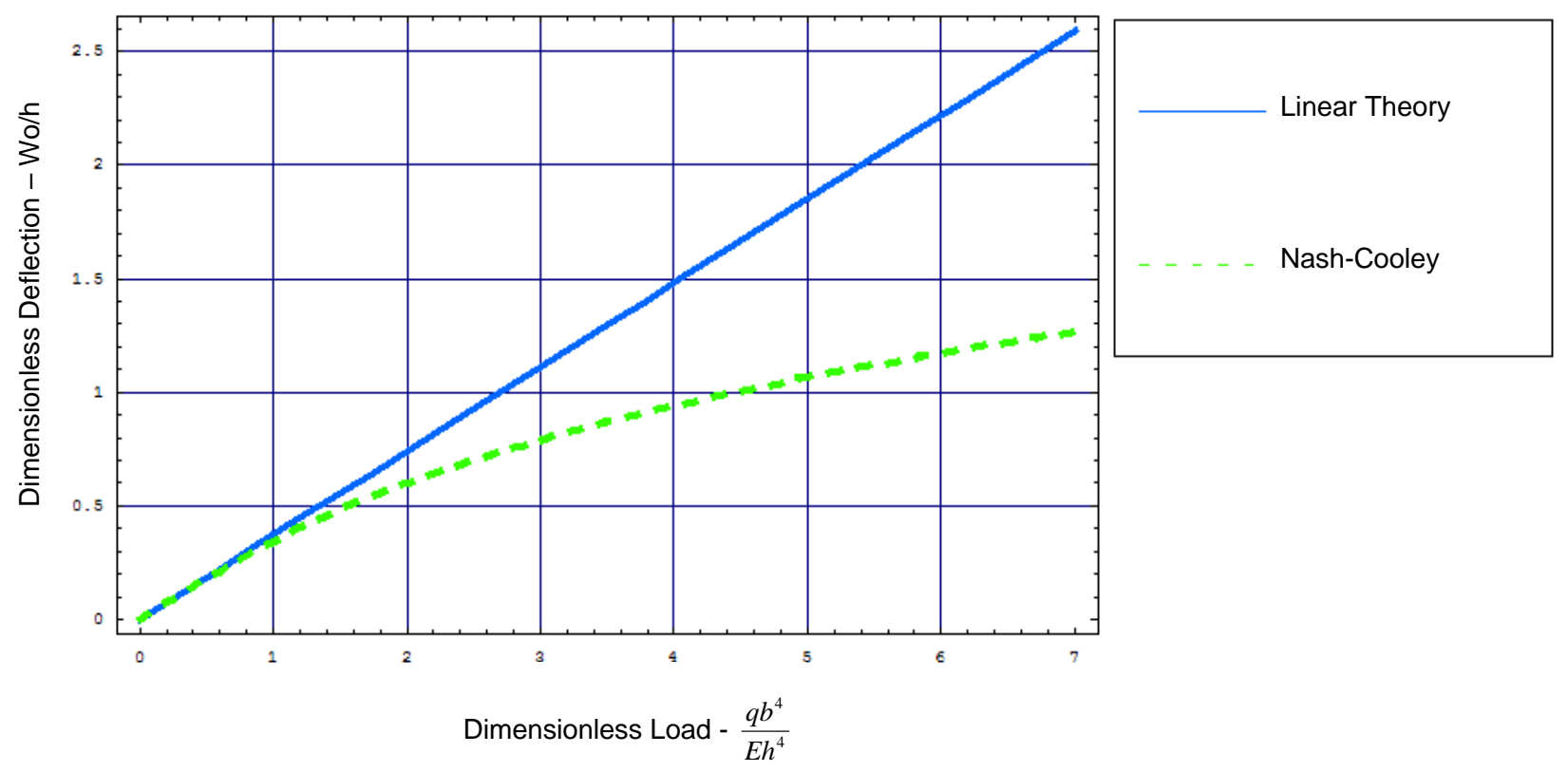

Figure 3.6: Nash and Cooley dimensionless deflection versus load results as compared to linear deformation theory; Note: Results presented are for an elliptical plate with a major to minor axis ratio of $2: 1, h \equiv$ thickness, $b \equiv$ semiminor axis, and $E \equiv$ Young's Modulus 
them, progressively became larger.

\subsection{Development of a Finite Element Model for the Diaphragm Pressure Sensor}

ANSYS finite element modeling software was used to help understand how the diaphragm would behave for different geometries at a pressure load of $2.413 \mathrm{MPa}$ (350 psi). Bending, membrane stiffness, and large deformation were all accounted for in the Shell181 elements used in the model. An input file for the ANSYS software was created to make the geometry, mesh, apply the loading and boundary conditions, and implement the solver. Postproccessing was also included to retrieve results such as axial strain along a path and deflection at various points. The input file was written in such a way so that it would loop through different ellipse like diaphragm configurations. To simplify the model both $\mathrm{x}$ and y-axis symmetry was used, Fig. 3.7 illustrates the quarter model of the elliptical diaphragm with the applied loading and boundary conditions.

Geometric nonlinear checking was also implemented in the input file. Upon receiving the displacement solution, the code would check for large deformations (transverse displacement $>50 \%$ diaphragm thickness), if this condition was found, 


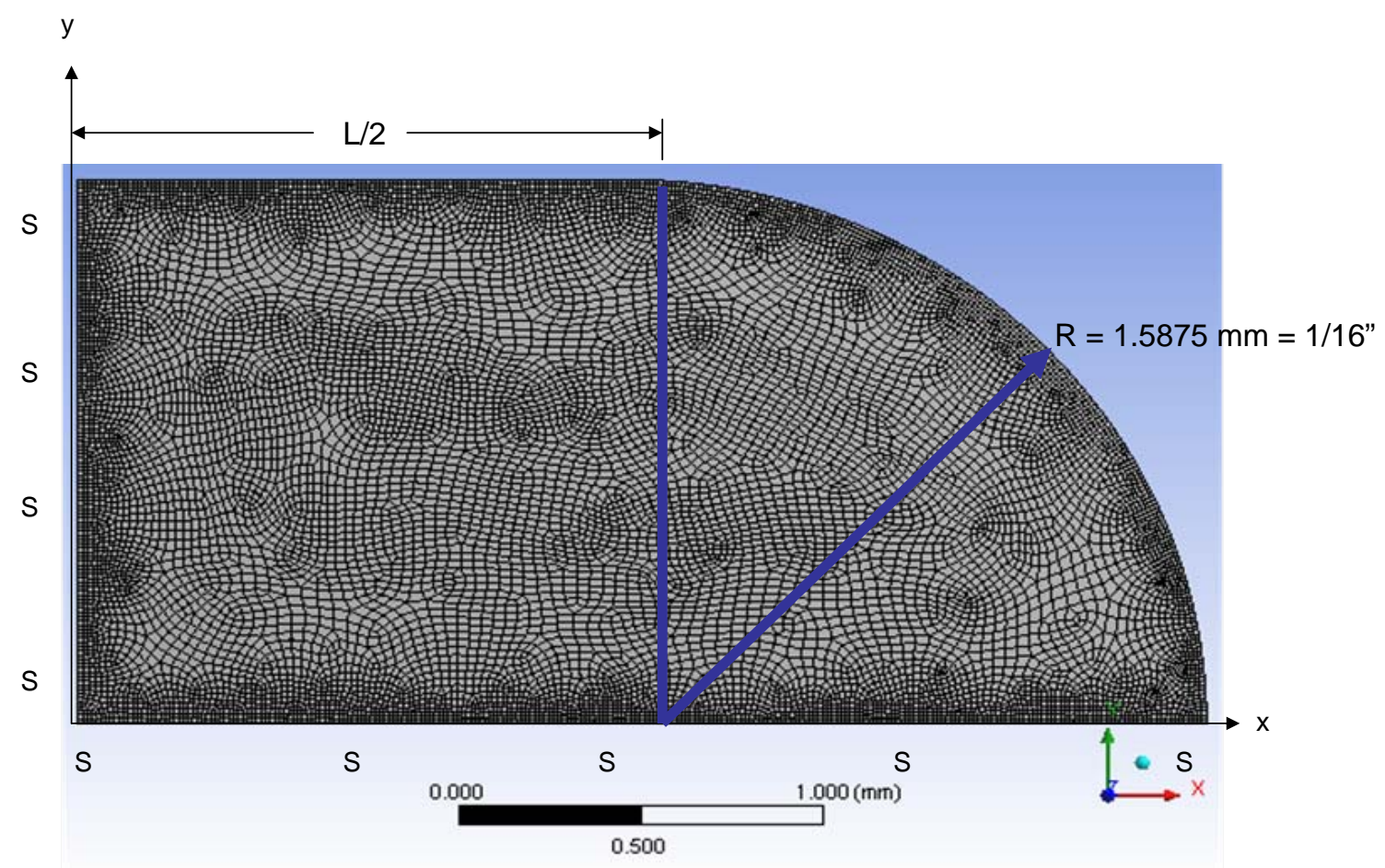

Figure 3.7: Diaphragm and loading conditions created by input file, $\mathbf{S}$ indicates symmetry boundary conditions were implemented along the depicted lines. The remaining lines were clamped. 
the finite element simulation would recommpute the solution again but with large deformation effects included in the shell elements, see [28] for theoretical details.

\subsubsection{Verification of the Finite Element Model}

To verify that the input file to the finite element analysis was written correctly, results for both linear and nonlinear regimes were compared to the large deformation solution of Nash and Cooley [34]. Deflection and strain results along the semimajor

axis were compared for dimensionless loads of, $\frac{q b^{4}}{E h^{4}}=0.157$ and $\frac{q b^{4}}{E h^{4}}=2$. Using [34], a comparable solution was derived using Mathematica for a major to minor axis ratio of 1.94 ( $a=3.0875 \mathrm{~mm}, b=1.5875 \mathrm{~mm})$. The convergence between the two methods can be seen in Fig. 3.8. It can be noticed that there is some discrepancy in the axial strain at the boundary in the nonlinear regime.

\subsection{Optimizing Diaphragm Geometry}

As described above, we took an iterative approach to explore the effects of the diaphragm dimensions on the resulting strain distribution in order to quickly zero in on recommended dimensions for prototype construction. Both the thickness(h) and the length(L) of a diaphragm (Fig. 3.7) were varied, and the central longitudinal and transverse strains were calculated at a target operating pressure of $2.413 \mathrm{MPa}(350$ 
Transverse Deflection vs. Position, For a Thin Eliptical Plate in the Linear and Nonlinear Deformation Regimes

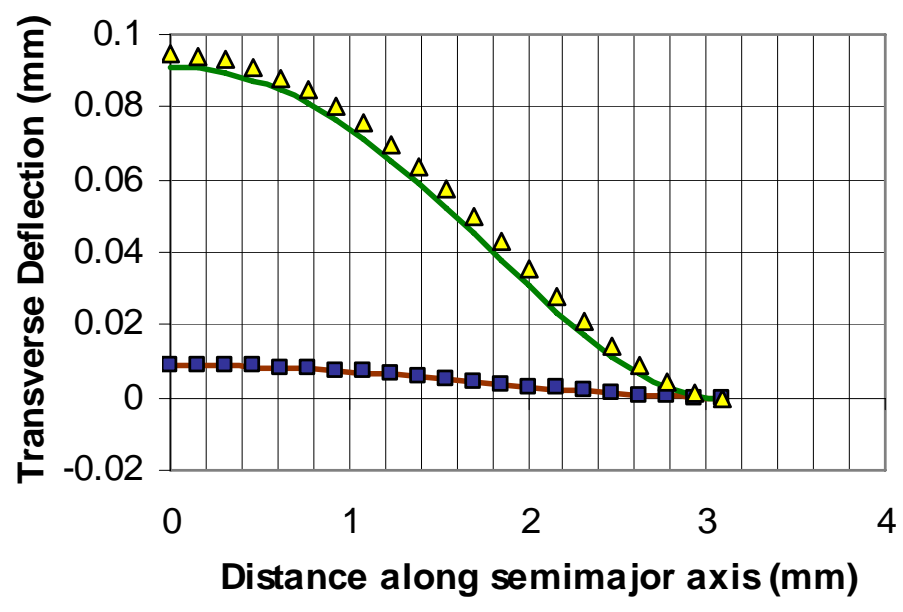

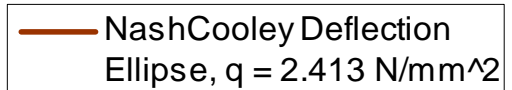

口 FEM Deflection via Input File, $\mathrm{q}=2.413 \mathrm{~N} / \mathrm{mm}^{\wedge} 2$ NashCooley Deflection, $\mathrm{q}=30.7679 \mathrm{~N} / \mathrm{mm}^{\wedge} 2$

$\triangle$ FEM Deflection via Input File, $\mathrm{q}=30.7679 \mathrm{~N} / \mathrm{mm}^{\wedge} 2$

(a) Transverse deflection verification

Axial Strain vs.Position, For a Thin Eliptical Plate in the Linear and Nonlinear Deformation Regimes

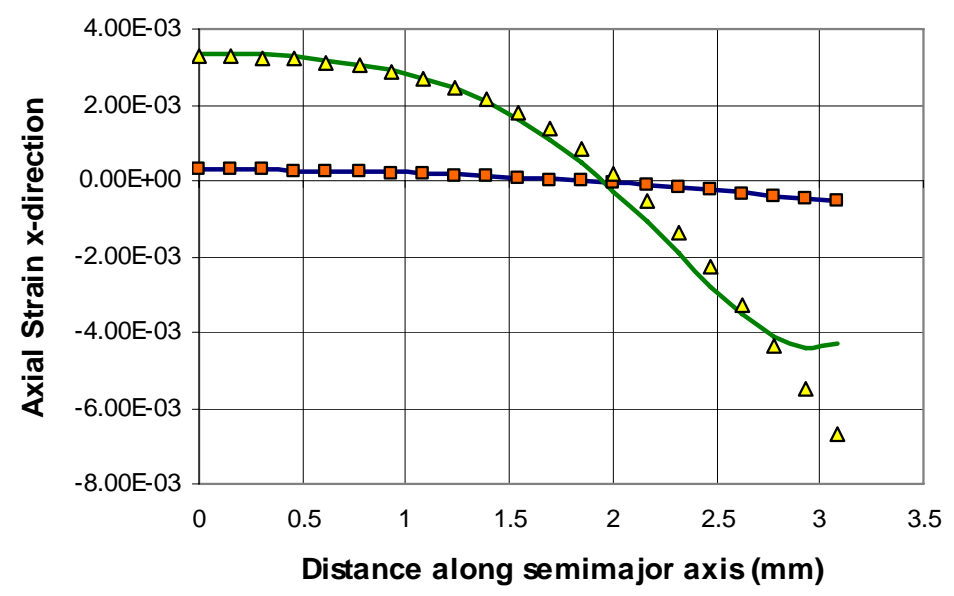

NashCooley Strain, q=2.413 N/mm²

․ FEM Strain via Input File, $\mathrm{q}=2.413$ $\mathrm{N} / \mathrm{mm}^{\Upsilon 2} 2$

NashCooley Strain, $q=30.7679 \mathrm{~N} / \mathrm{mm}^{\wedge} 2$

$\Delta \quad$ FEM Strain via Input File, $q=30.7679$ $\mathrm{N} / \mathrm{mm}^{\uparrow} 2$

(b) Axial Strain Verification

Figure 3.8: Verification of input file with analytical results 
Table 3.1: Summary of Axial Strain Results from Simulation; Longitudinal strain (xdirection) at the center of a rounded end diaphragm with 23 bar of pressure [350 psi] applied on the opposite side are shown as a function of varied diaphragm geometry. Strain values in bold achieved at least $200 \mu \epsilon$ of tensile strain without exceeding the yield strain for stainless steel $(1500 \mu \epsilon)$. Notice that for some thicker and longer diaphragms, the strain at the center dropped significantly and even became weakly compressive in the longitudinal direction. Figure 3.7 explains this behavior in more detail.

\begin{tabular}{|c|c|c|c|c|}
\hline Thickness: & $\begin{array}{c}50 \mu \mathrm{m} \\
{[2 \mathrm{mils}]}\end{array}$ & $\begin{array}{c}100 \mu \mathrm{m} \\
{[4 \mathrm{mils}]}\end{array}$ & $\begin{array}{c}150 \mu \mathrm{m} \\
{[6 \mathrm{mils}]}\end{array}$ & $\begin{array}{c}200 \mu \mathrm{m} \\
{[8 \mathrm{mils}]}\end{array}$ \\
\hline Length & & & & \\
\hline $2 \mathrm{~mm}$ & $\mathbf{1 . 2 1 5 e - 3}$ & $\mathbf{7 . 3 6 e - 4}$ & $\mathbf{3 . 2 0 e - 4}$ & $-1.76 \mathrm{e}-4$ \\
$5 \mathrm{~mm}$ & $\mathbf{2 . 4 5 e - 4}$ & $9.140 \mathrm{e}-5$ & $3.97 \mathrm{e}-5$ & $2.07 \mathrm{e}-5$ \\
$8 \mathrm{~mm}$ & $8.67 \mathrm{e}-5$ & $5.47 \mathrm{e}-6$ & $-5.1 \mathrm{e}-8$ & $-5.51 \mathrm{e}-7$ \\
$11 \mathrm{~mm}$ & $3.45 \mathrm{e}-5$ & $2.88 \mathrm{e}-6$ & $-1.25 \mathrm{e}-7$ & $-2.755 \mathrm{e}-7$ \\
\hline
\end{tabular}

psi). The results from the input file, in Table 2 and Table 3 , suggested that $150 \mu \mathrm{m}$ was the ideal diaphragm thickness. This is because, at this thickness, the strain in both the $\mathrm{x}$ and $\mathrm{y}$ directions are greater than the target $(200 \mu \epsilon)$ but less than the yield strain of stainless steel $(1500 \mu \epsilon)$.

The input file was then modified to create a more detailed study of the longitudinal strain distribution in a diaphragm with a fixed thickness of $150 \mu \mathrm{m}$, the axial strain results for varying lengths, $\mathrm{L}$ is shown in Fig. 3.7. The ideal strain distribution is a reasonably constant tensile strain over a distance of 3 to $4 \mathrm{~mm}$, which is the gauge length of optical fiber using either Rayleigh scattering or FBGs. Thus, the 
Table 3.2: Summary of Transverse Strain Results from Simulation; Transverse strain (y-direction) at the center of a rounded end diaphragm with 23 bar of pressure [350 psi] applied on the opposite side. According to the finite element simulations, the ideal diaphragm thickness was near $150 \mu m$ [6 mils], so that 23 bar of pressure [350 psi] produced at least $200 \mu \epsilon$ of tensile strain (shown in bold) without exceeding the yield strain for stainless steel $(1500 \mu \epsilon)$.

\begin{tabular}{|c|c|c|c|c|}
\hline Thickness: & $\begin{array}{c}50 \mu \mathrm{m} \\
{[2 \mathrm{mils}]}\end{array}$ & $\begin{array}{c}100 \mu \mathrm{m} \\
{[4 \mathrm{mils}]}\end{array}$ & $\begin{array}{c}150 \mu \mathrm{m} \\
{[6 \mathrm{mils}]}\end{array}$ & $\begin{array}{c}200 \mu \mathrm{m} \\
{[8 \mathrm{mils}]}\end{array}$ \\
\hline Length & & & & \\
\hline $2 \mathrm{~mm}$ & $4.368 \mathrm{e}-3$ & $2.487 \mathrm{e}-3$ & $\mathbf{1 . 0 9 e - 3}$ & $\mathbf{6 . 0 4 e - 4}$ \\
$5 \mathrm{~mm}$ & $4.669 \mathrm{e}-3$ & $2.972 \mathrm{e}-3$ & $\mathbf{1 . 3 3 e - 3}$ & $\mathbf{7 . 3 8 e - 4}$ \\
$8 \mathrm{~mm}$ & $4.076 \mathrm{e}-3$ & $2.975 \mathrm{e}-3$ & $\mathbf{1 . 3 4 e - 3}$ & $\mathbf{7 . 4 1 e - 4}$ \\
$11 \mathrm{~mm}$ & $4.722 \mathrm{e}-3$ & $2.970 \mathrm{e}-3$ & $\mathbf{1 . 3 4 e - 3}$ & $\mathbf{7 . 4 0 e - 4}$ \\
\hline
\end{tabular}

target strain distribution is about $200 \mu \epsilon$ when the diaphragm length is 3 or $4 \mathrm{~mm}$.

Since, the geometry of the diaphragm Fig. 3.7 is predominantly a square plate, to see if the results in Fig. 3.9 made sense, the interest lengths just found $(L=3 \mathrm{~mm}$ and, $L=4 \mathrm{~mm}$ ) were compared to the linear solution by Kantorovich [27] for a clamped square plate subjected to uniform pressure. As shown in Fig. 3.10, the trend is the same, with the square plate solution yielding a slightly more strain distribution throughout.

Note: the input files for the above analysis can be found in Appendix C. 
Simulated Strain Distribution on the Inside of a $150 \mu \mathrm{m}$ Thick, 3mm Wide,

304 Stainless Steel Diaphragm with 23 bar of Pressure Applied from the Outside

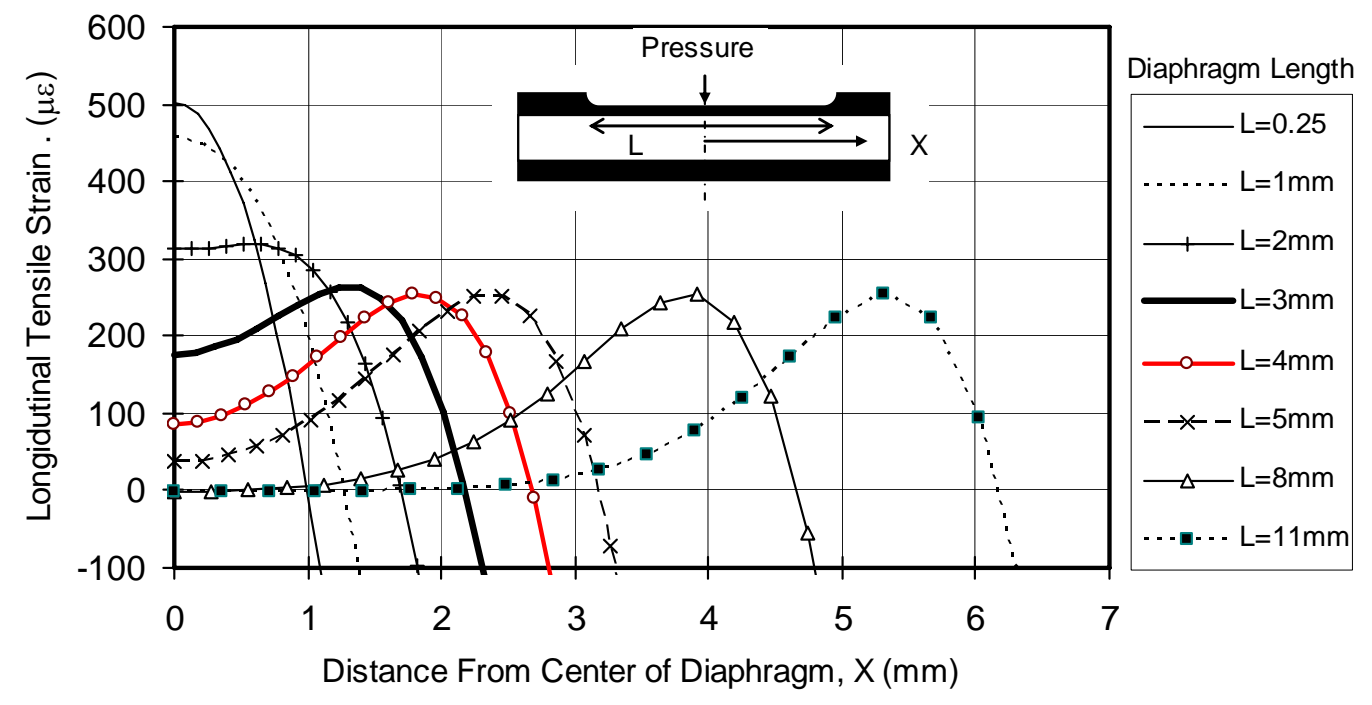

Figure 3.9: Axial Strain distribution for varying lengths; Modeled strain distributions in diaphragms of various lengths made by machining a flat rectangular diaphragm into square cross-section stainless steel tubing. For a 24.13 bar [350 psi] pressure sensor based on Rayleigh scattering in an optical fiber, the target strain distribution is about $200 \mu \epsilon$ with minimal fluctuation over $3-4 \mathrm{~mm}$. The desired behavior is approximated when the diaphragm length is 3 or $4 \mathrm{~mm}$. 

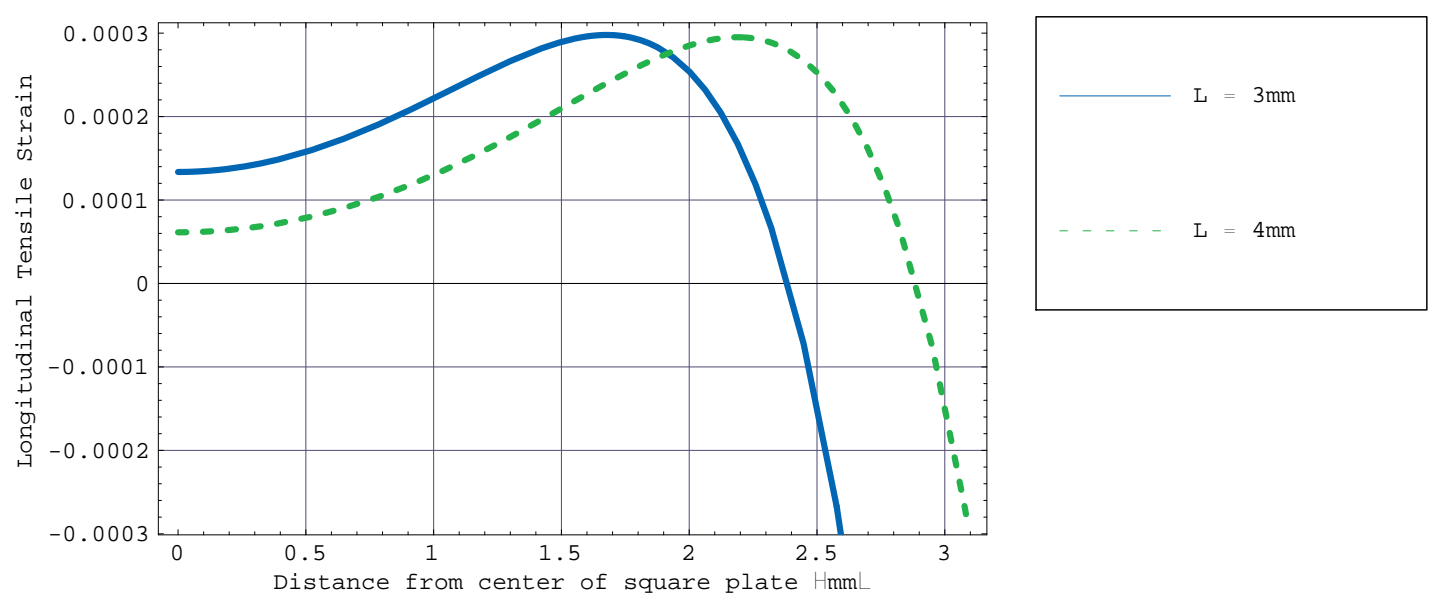

Figure 3.10: Axial Strain distribution for a square plate under uniform pressure; Variation of Longitudinal tensile strain as a function of distance from the mid-point for a clamped square plate subjected to 24.13 bar [350 psi] and the half width $\equiv b \equiv$ $1.5875 \mathrm{~mm}$.

\subsection{Summary and Conclusions}

In this chapter, a new diaphragm design, allowing easy fabrication as compared to the bellows design, was introduced. Commercially available, general-purpose finite element software, ANSYS, was used to gain experience with the software as well as to analyze arbitrary plates under transverse pressure. Results were compared with analytical, geometric nonlinear, results available for an elliptical plate. The agreement of both solutions show that the input file was written correctly. Using the input file, optimized diaphragm dimensions were found such to yield our desired strain distribution. The final design includes a stainless steel square tube with a thin 
flat milled section producing the diaphragm, see Fig. 3.4. Chapter 4 introduces the fiber-optic bonding scheme and its finite element formulation. 


\section{Chapter 4}

\section{Numerical Simulation of}

\section{Diaphragm Design}

\subsection{Overview}

This chapter focuses on the modeling of the square tube diaphragm sensor design. In this model the fiber and adhesive elements are added in addition to the stainless steel square tube. Two feasible fabrication techniques for the bonding (adhesion) of the fiber to the bottom of the diaphragm were suggested by the fiber-optic experts at Luna Innovations. Each of the two approaches were modeled in ANSYS 10 workbench to approximate the axial strain through the core of the copper coated fiber. The fiber 
is coated with a thin film of copper to allow for proper adhesion to take place with silver. Again, symmetry was used and a quarter model of the system was meshed and results were calculated. Details of the model are discussed below. Figures 4.1 and 4.2, illustrate the front symmetry plane for the two bonding methods. One technique considers using a plate of silver epoxy as the main bonding agent. More details of the bonding procedure will be given in Chapter 5 . The other technique uses a hemisphere rather than a plate of the Ag epoxy, surrounding the fiber completely (Fig. 4.2).

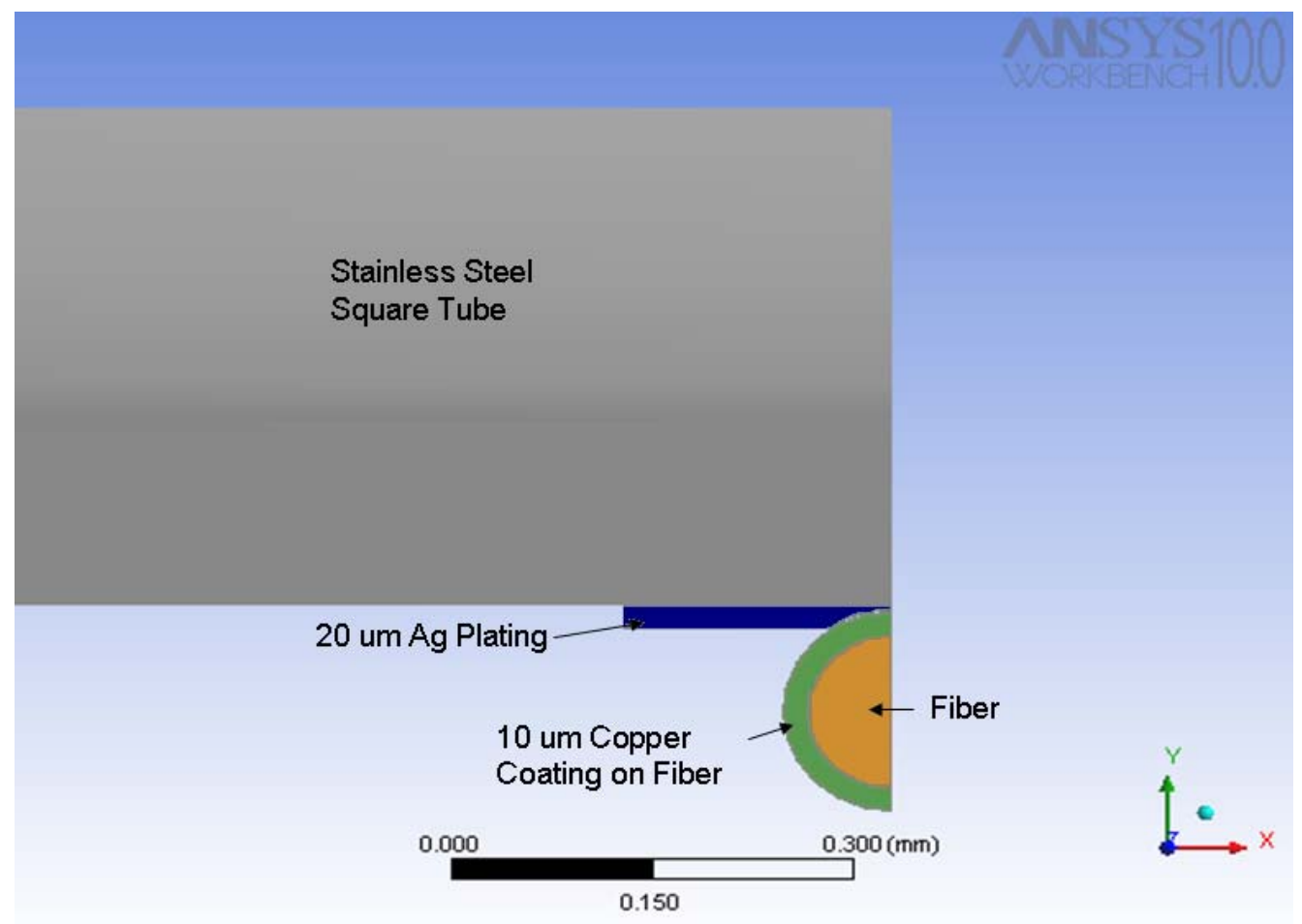

Figure 4.1: Silver plate bonding technique 


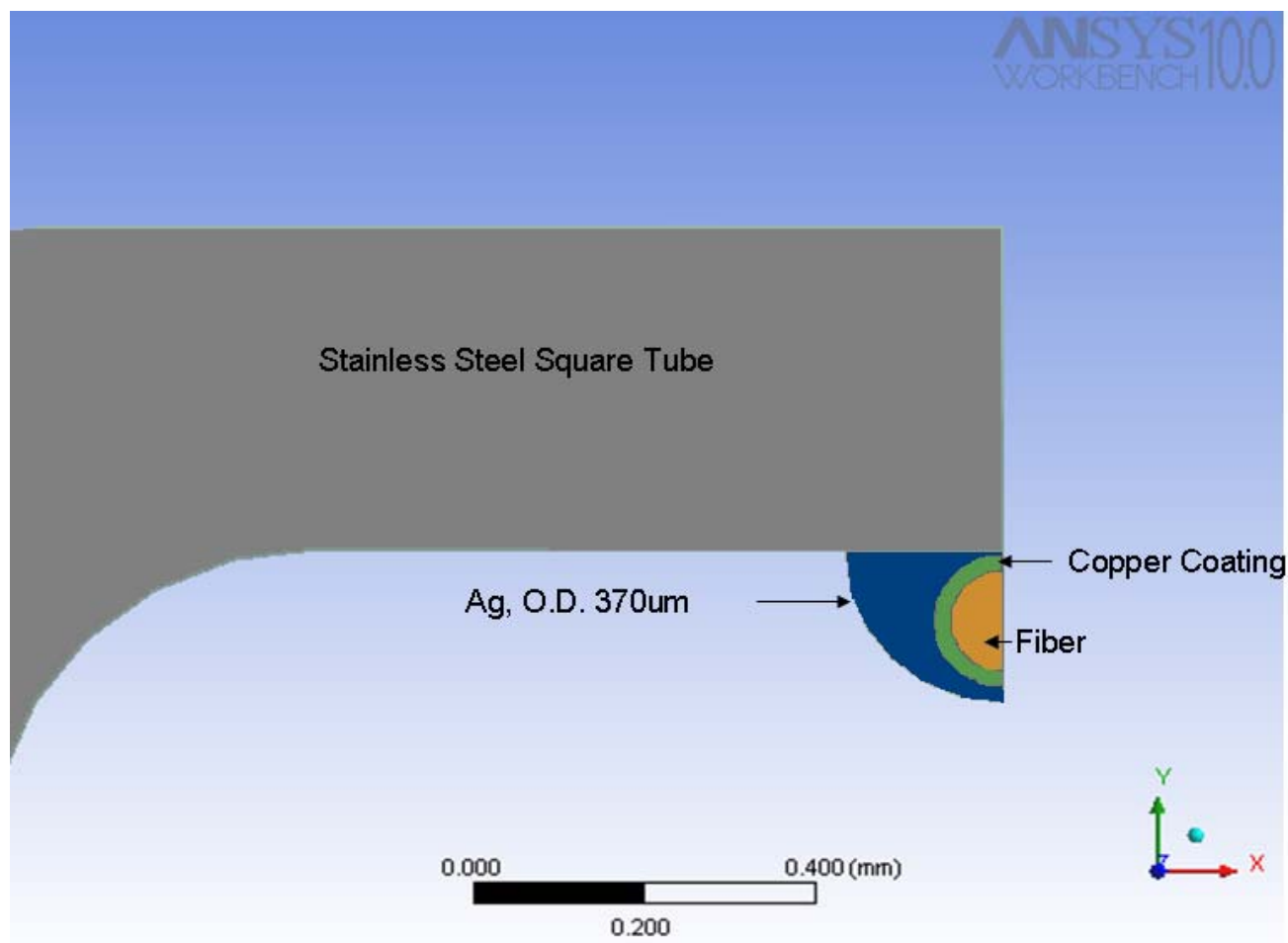

Figure 4.2: Silver hemisphere bonding technique 


\subsection{Square Tube Diaphragm Design Finite Ele- ment Model}

The fiber is to be bonded longitudinally and centered directly underneath the diaphragm face of the stainless steel tube, with the hope that the strain distribution in the fiber will resemble that of the results in Fig. 3.9. Four different bonding scenarios, two for each bonding technique (plate/hemisphere), were simulated in order to decide on the best configuration. In method one, the entire length of the fiber is bonded to the stainless steel tube, while method two restricts the bonding to directly underneath the diaphragm section only. The general loading and boundary conditions for the model is illustrated in Fig. 4.3. The model was made $10 \mathrm{~mm}$ long so that the large stress gradients generated at the fixed edge support would not interfere with the results in the area of interest (diaphragm section).

\subsubsection{Finite Element Model Description}

Each of the four models described above were meshed using the same elements, Solid187, Solid186, Conta174, and Targe170. The total number of nodes ranged from 120,000 to 128,000 and the average CPU time for a single processor machine was 15 minutes. The mesh was deemed sufficient by the structural error tool available 


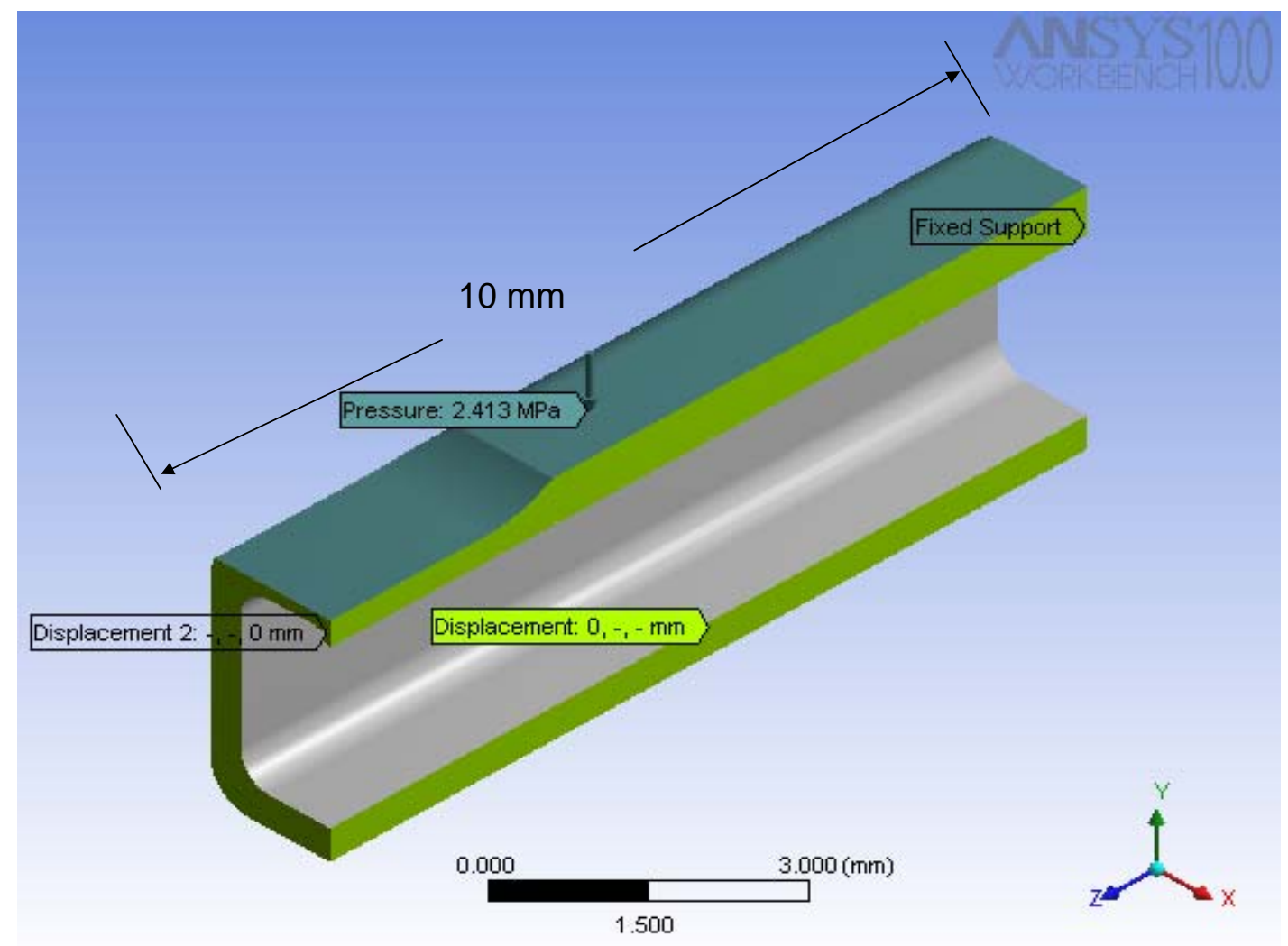

Figure 4.3: Loading and boundary conditions for square tube mill design 
in ANSYS workbench 10. This tool uses an energy approach to give an estimate of error at each element, if the maximum energy value at an element goes above $10^{-5} \mathrm{~mJ}$. It is advised to refine the mesh at that area. Typically the maximum error of any element found in this analysis was on the order of $10^{-7}$.

Solid187 is a higher order 3-D tetrahedron element with 10 nodes and three degrees of freedom at each node (translation in the $\mathrm{x}, \mathrm{y}, \mathrm{z}$ ). This element is well suited for modeling irregular meshes, such as encountered in milled portion (diaphragm) of the stainless steel tube. Its capabilities include: large deflection, large strain, plasticity, stress stiffening, hyperplasticity, and creep. It also has the ability to simulate the deformed shape of nearly incompressible elastoplastic materials, and fully incompressible hyperelastic materials. Solid186 is a higher order 3-D solid element with 20 nodes and three degrees of freedom at each node (translation in the $\mathrm{x}, \mathrm{y}, \mathrm{z})$. It offers the same special features as Solid18\%.

Conta174 is a 3-D surface to surface contact element with eight nodes. It is used to represent sliding between 3-D target surfaces (Targe170) and a deformable surface. This element is located on solid/shell elements with midside nodes, i.e. Solid186, Solid18\%...etc, and has the same geometric characteristics as that of the element face it is connected to. Contact occurs when the contact element penetrates the target surface. The target surface is discretized by a set of target elements, 
Table 4.1: Material Properties, Diaphragm Model. $\mathrm{E}=$ Young's Modulus, $\nu=$ Poisson's Ratio, $\sigma_{y}=$ Yield Stress, $\alpha=$ Coefficient of Thermal Expansion

\begin{tabular}{|c|c|c|c|c|}
\hline Material & $\mathrm{E}(\mathrm{GPa})$ & $\nu$ & $\sigma_{y}(\mathrm{MPa})$ & $\alpha\left(\frac{\mu \epsilon}{{ }^{\circ} \mathrm{C}}\right)$ \\
\hline Glass Fiber & 72.4 & 0.2 & $152.2-167.8$ & 0.5 \\
\hline Silver & 72 & 0.37 & $45-65$ & $19.5-19.9$ \\
\hline Stainless Steel & 193 & 0.25 & $170-310$ & $15-18$ \\
\hline Copper & 110 & 0.34 & 33.3 & 16.5 \\
\hline
\end{tabular}

Targe170. Targe170 is a 3-D element used to represent various surfaces associated with certain contact elements. Refer to [28] for more details on ANSYS elements. Table 4.1 shows the linear elastic material properties defined in each model.

Since other parts were added to the finite element model, i.e. the copper coated fiber and silver epoxy, contact regions were defined using Conta174 and Targe170 elements described above. In ANSYS workbench, each contact region can be described in one of five ways: bonded, frictional, frictionless, rough, or no separation. Because of the differences in bonding methods one and two, described above, ANSYS contact options "bonded" and "frictionless" were used. The "bonded" option enforces no sliding or separation between faces or edges and allows for a linear solution, since the contact length/area will not change during application of a load [37]. This option accurately models the fiber attachment described by method one. For the fiber attachment described in method two, the "frictionless" option was used to describe the behavior between the copper coated fiber and stainless steel wall at regions away 
from the diaphragm. The "frictionless" option allows free sliding between faces. This solution is nonlinear because the contact area changes with the deformation [37]. A pictorial view of bonding method one is shown in Fig. 4.4. It can be noticed here that all regions are defined as bonded, meaning that the surfaces are not allowed to slide with respect to each other in the model, more specifically the displacements at the bonding interfaces are the same. In method two, if the copper coated fiber were to come into contact with inside wall of the stainless steel, they would be able to slide freely against one another.

\subsubsection{Finite Element Results}

The axial strain simulation results through the center of the fiber are depicted in the following figures. The simulation results for the bonding techniques defined by method two, are shown in Figs. 4.5 and 4.6. Only one case for the method one technique is shown, Fig. 4.7. For the cases where the bond is restricted to only $2 \mathrm{~mm}$ (method two), there is very little compressive strain generated beyond the diaphragm region. This is in contrast to the other case, Fig. 4.7. The large compressive strain, Fig. 4.7, has the potential to negatively influence the desired tensile strain. Thus, the configurations shown in Figures 4.5 and 4.6 are more desirable.

The strain curve versus position trend for each case is as expected, recall the 


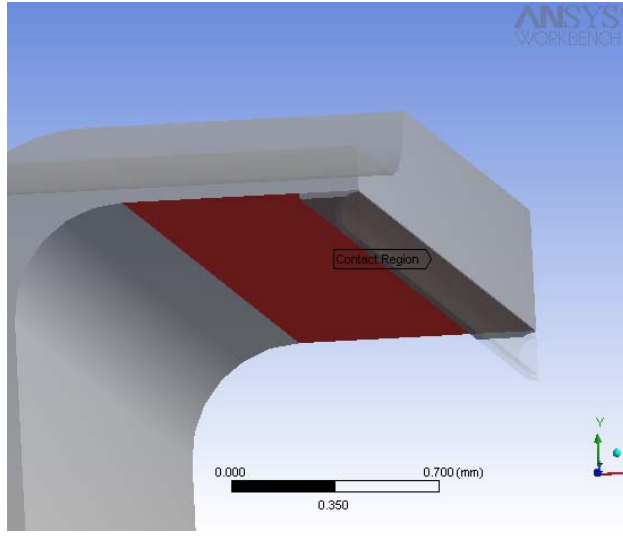

a)

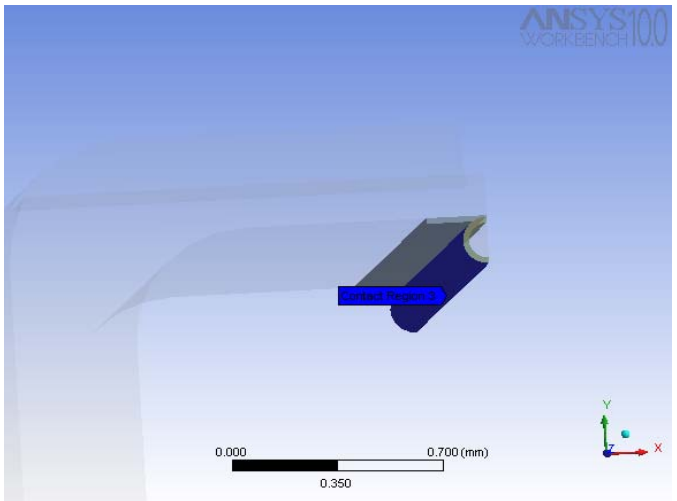

c)

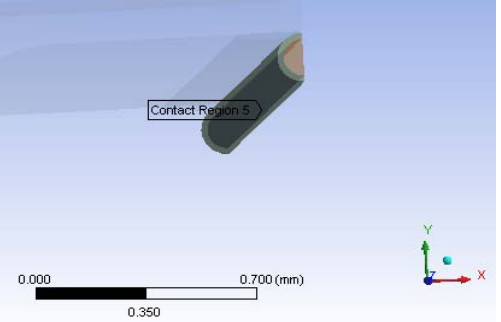

b)

a) Between Tube Wall and $\mathrm{Ag}$ ANSYS Status: BONDED

b) Between The inner surface of copper coating and outer surface of the fiber ANSYS Status: BONDED

c) Between The outer surface of the copper and Ag ANSYS Status: BONDED

If contact regions are BONDED, then no sliding or separation between faces or edges is allowed

Figure 4.4: Contact regions between parts 


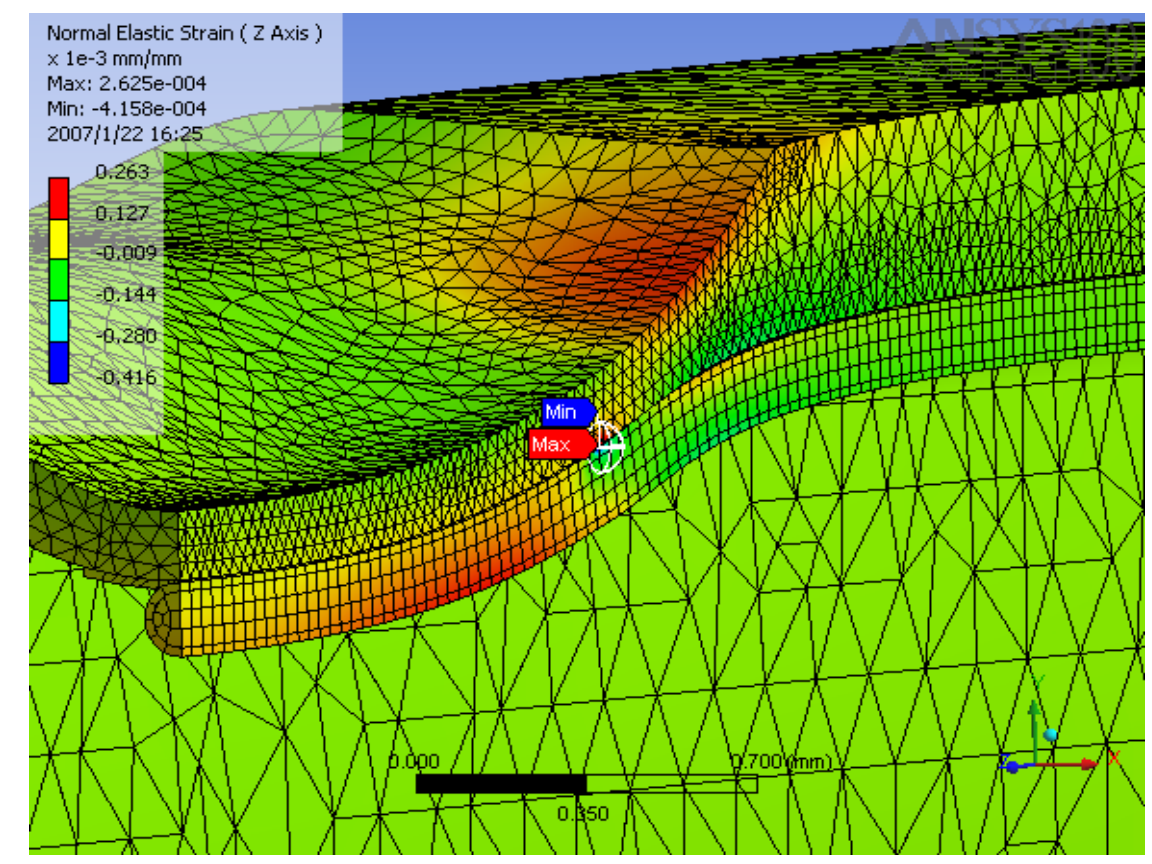

(a) ANSYS axial strain elemental contour plot and deformed shape

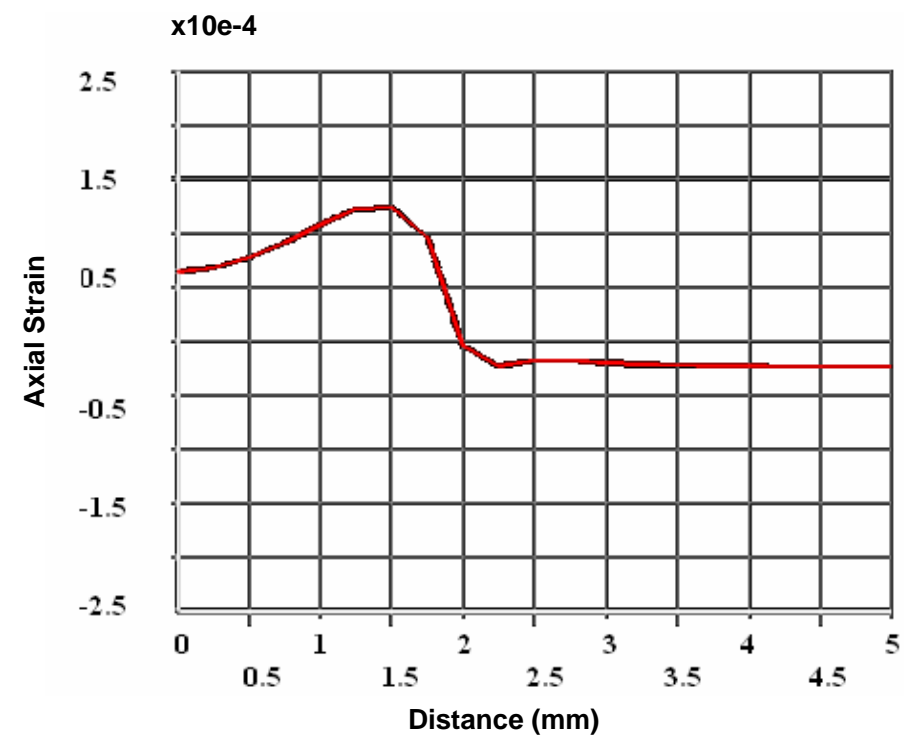

(b) Axial strain through the center of the fiber, beginning at the midpoint

Figure 4.5: ANSYS axial strain results for a plate bonded structure using method two, Applied Pressure: $2.413 \mathrm{MPa}$ [350 psi] 


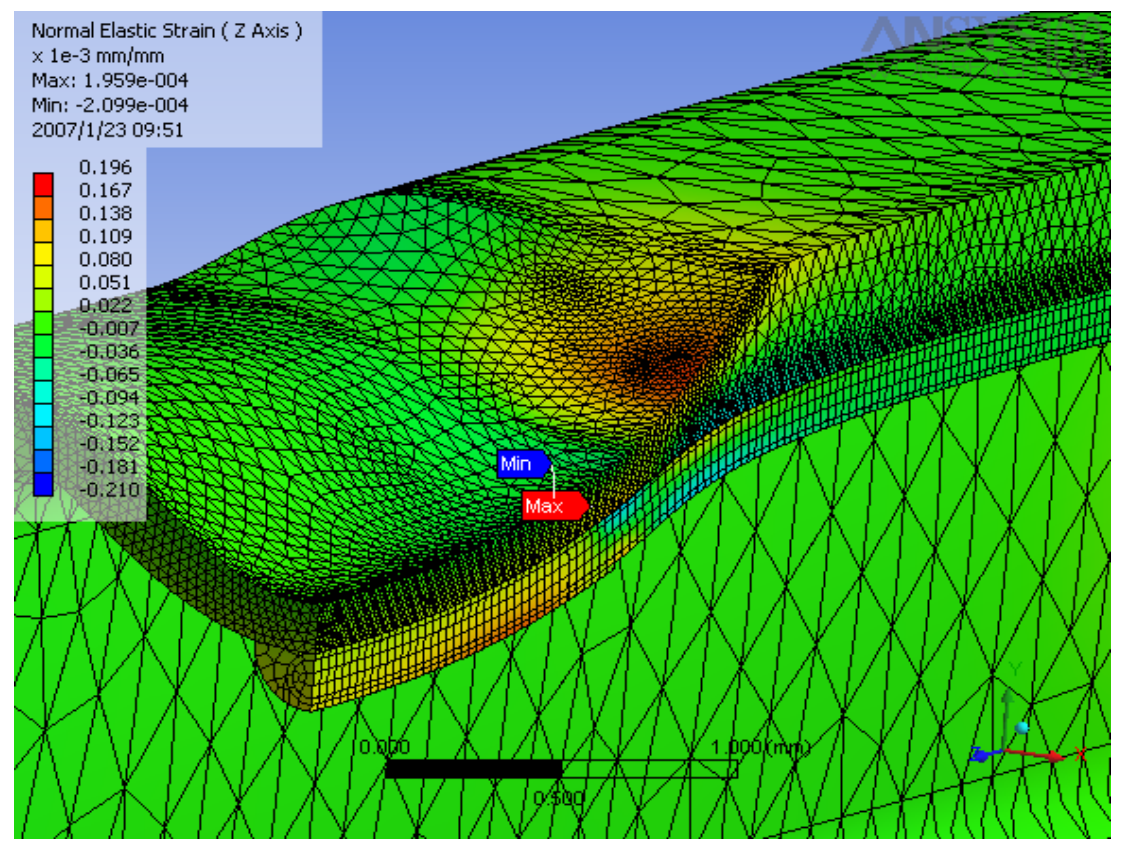

(a) ANSYS axial strain elemental contour plot and deformed shape

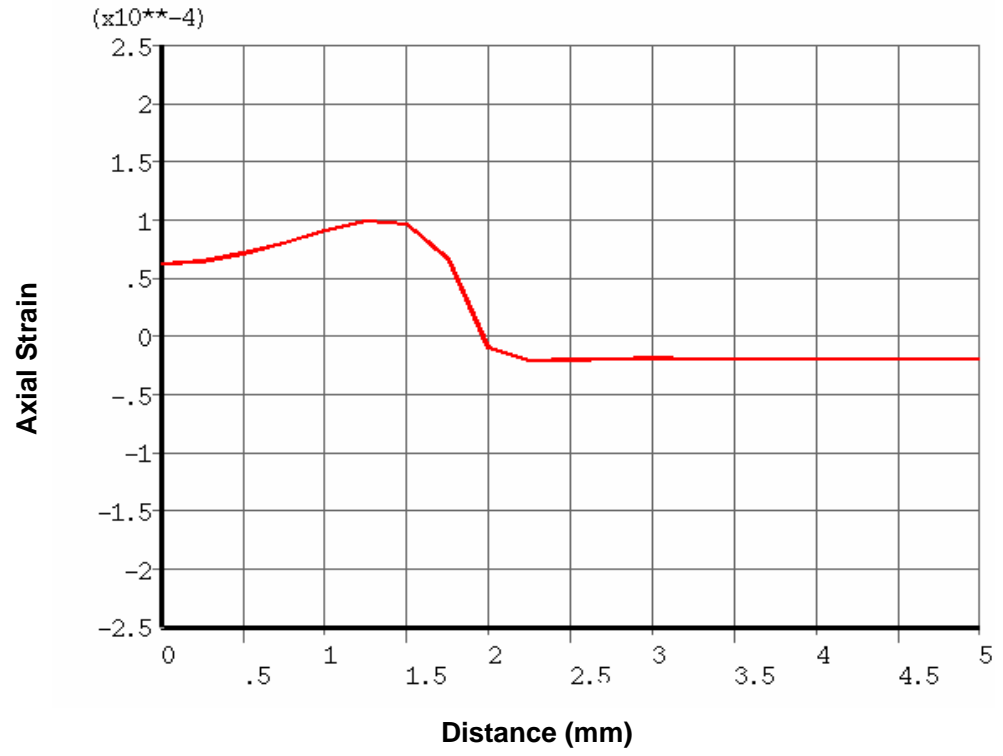

(b) Axial strain through the center of the fiber, beginning at the midpoint

Figure 4.6: ANSYS axial strain results for a hemisphere bonded structure using method two, Applied Pressure: 2.413 MPa [350 psi] 


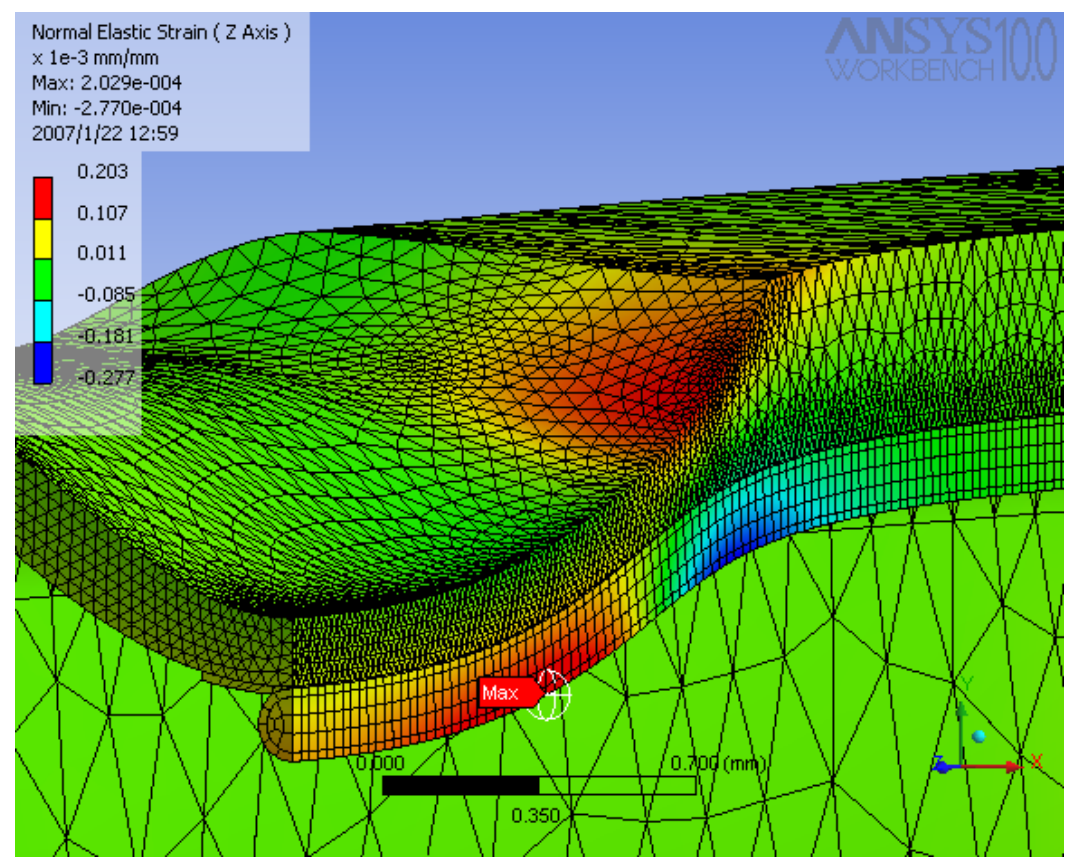

(a) ANSYS axial strain elemental contour plot and deformed shape

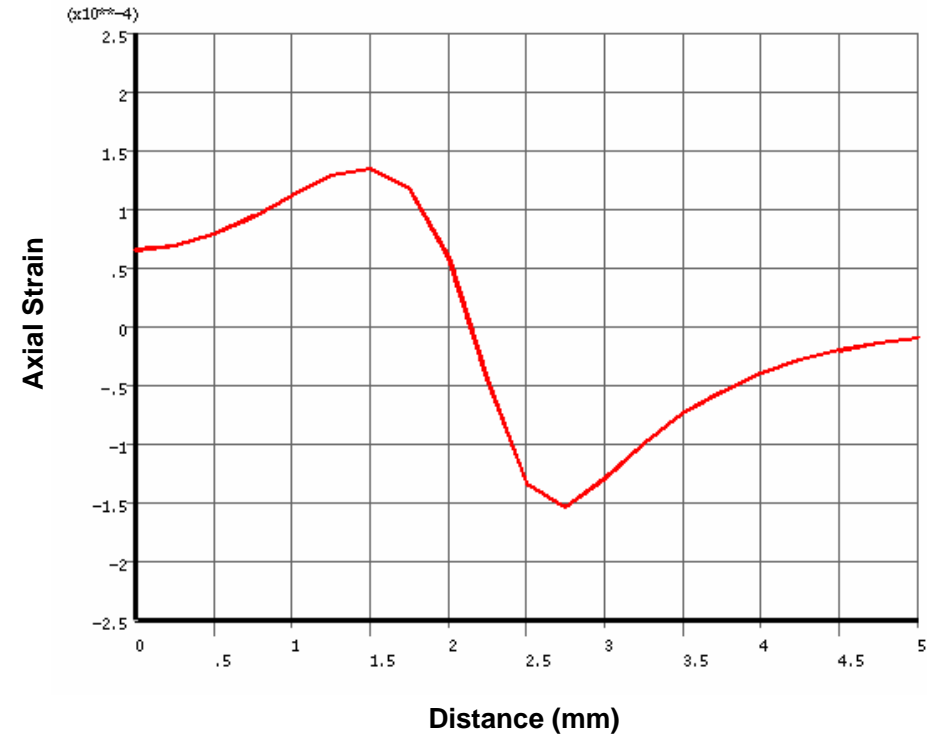

(b) Axial strain through the center of the fiber, beginning at the midpoint

Figure 4.7: ANSYS axial strain results for a plate bonded structure using method one, Applied Pressure: 2.413 MPa [350 psi] 
earlier development of a thin diaphragm (Fig. 3.9), i.e. the central axial strain of a clamped elliptical diaphragm subject to uniform pressure is not maximum for large semimajor axis to semiminor axis ratios. It can also be noticed that it is apparent that the geometry of the bonding agent Ag directly effects the strain results. This can be seen from Figures 4.5 and 4.6. The peak strain for the flat plate bonding technique yields $125 \mu \epsilon$ while the hemisphere bonding technique yields $100 \mu \epsilon$. This is most likely due to the amount of material stiffness in both cases. However, to ensure a more secure bond, a near hemisphere bonding style was used in the fabrication of a prototype sensor. It is worth noting that for the current room temperature simulation, the von-Mises stress was well below the yield stress of each material. However, at the boundary of the diaphragm the von-Mises stress was maximum. Thus, thinner diaphragms $(<150 \mu m)$ are likely to plastically deform when using stainless steel.

A prototype sensor was fabricated based off the recommended dimensions established in Chapter 3, Fig. 4.8. 


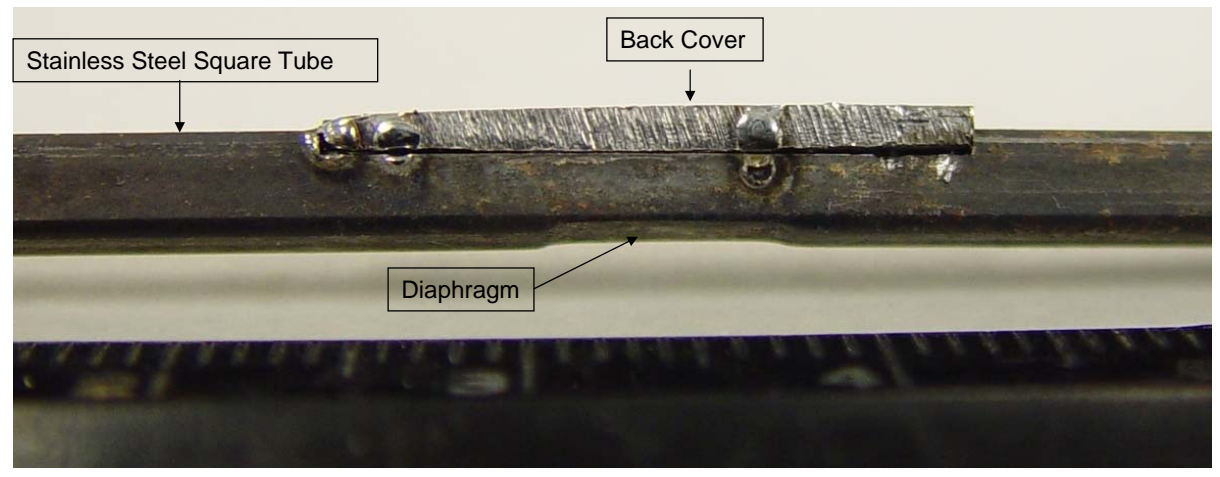

Figure 4.8: Prototype, diaphragm design, after fabrication. Face opposite to diaphragm was removed for the bonding of the fiber. A back cover was used to seal the opening.

\subsection{Square Tube Diaphragm Design Preliminary Testing Results}

Plans to test the sensor at room temperature at pressures up to 500 psig were made. Unfortunately, the sensor was accidentally broken while being photographed. The testing results were not really that necessary, because we would be validating finite element results at room temperature. We feel quite confident that the finite element results described in this chapter are correct as they resemble that of the validation results discussed in Chapter 3. Validation using experimental testing will become important when finite simulations become highly nonlinear due to plasticity and creep effects. This scenario becomes apparent in the next phase of design, sustained pressure measurement at $800^{\circ} \mathrm{C}$, and is the topic of the next chapter. 


\section{Chapter 5}

\section{Conclusions and Future Work}

\subsection{Discussion}

Monitoring accurate temperature and pressure profiles in harsh environments is currently in high demand in aerospace, gas turbine engines and nuclear reactor simulators. The work performed in this thesis is for NASA's SAFE100, a simulator being developed to supplement the introduction of early flight fission. The idea is, that by properly characterizing the thermo-mechanical activity within non-nuclear reactor simulators, nuclear operation can be more accurately controlled and confidence in thermo-mechanical simulations will be high. However, the ability to characterize non-nuclear test core simulators is currently limited by the lack of instrumentation 
options available for distributing large numbers of sensors throughout the core to monitor these parameters in real-time. Previous literature shows that fiber-optical measurement techniques provide numerous advantages over electrical based devices. This fact is especially true for an array of sensors operating at temperatures approaching $800^{\circ} \mathrm{C}$.

In this thesis, we have proposed a distributed fiber-optics based sensor, with its largest planar dimension equal to $3.175 \mathrm{~mm}$. In Chapter Two, a simple design was initiated, involving a glass capillary tube enclosing a fiber optic cable, with end caps made from fused silica. We found that the external capillary tube acted as a somewhat effective transducing element. We found room for improvement and modified the design by incorporating two bellows. It was seen that the bellows design is about 7.6 times more effective than the initial design. A scaled model of the prototype was made to show proof of concept. The scaled bellows sensor was tested at room temperature for pressures up to 55 psig. The experimental results were not as good as we would have liked, due to a bonding failure, however it brought to light the complexities that would be involved with pressure and temperature sensors having small envelopes. It was then apparent if we were unsuccessful at 10x scale, it would be very difficult for the bellows design to be fabricated at much smaller dimensions. In light of this, a new design, one that eliminated complex bonding of 
many parts, was sought.

In Chapter Three, a diaphragm based pressure sensor design was presented, which effectively reduced the complexity associated with the bellows design. In this study, a square stainless steel tube was used as the base structure, a diaphragm of length L, and thickness h, was then created using a flat endmill. ANSYS finite element software was used in a parameter study to discover the dimensions of a diaphragm necessary to create a desired distribution of strain, $200 \mu \epsilon$ at $2.413 \mathrm{MPa}$ [350 psig] over 3 to $4 \mathrm{~mm}$. The source code (input file) written for the parameter study was verified through an elliptic plate example in both the linear and nonlinear regimes. Finite element results showed that the target distribution of strain could be realized for $L=3 m m, 4 m m$ and $h=150 \mu m$.

Chapter Four, presents the expected performance of the sensor at room temperature through a detailed finite element model. The model coalesces both the base structure and diaphragm with the fiber and bonding agents. Based on their experience with bonding fibers to metals, technicians from Luna Innovations recommended using a silver epoxy, and thus making it necessary to copper coat the fiber. The finite element simulations of the complete model agreed with the expected results of Chapter Three. We feel that the current design is cheap, easy to manufacture, and yields adequate results for the pressure portion of the design. Plans to operate this 
sensor at high temperatures is the topic of the next section.

\subsection{Future Work}

Thus far only the pressure transducer portion of this research has been studied with plans of implementation of a temperature discrimination technique discussed in Chapter One. We can easily add a reference fiber for use of temperature discrimination. We foresee many difficulties with the current design at high temperatures due to mainly the misfit strains generated due to thermal CTE differences between bonds. The fear is not only failure, which may or may not be an issue depending on material nonlinearity effects such as creep and stress relaxation as sometimes these phenomena tend to undo deformations due to geometric nonlinearities, see [38], but also signal wash out of our strain signal due to pressure, and long term reliability.

In the current bond configuration there is a broad range of CTE values throughout the bond layers, with the largest difference coming at the interface between the copper $\left(16.5 \frac{\mu \epsilon}{{ }^{\circ} \mathrm{C}}\right)$ and the fiber $\left(0.5 \frac{\mu \epsilon}{{ }^{\circ} \mathrm{C}}\right)$. We are interested in learning the deformation/stress state of the bond after it has cooled. In the bonding procedure, initially, all components are approximately at $800^{\circ} \mathrm{C}$ and a thin silver layer is applied to the under side of the diaphragm. The copper coated fiber is subsequently attached and then more silver is added over the fiber to form a hemispherical shape, refer to Fig. 
4.2. The structure is then allowed to cool to the room temperature. Because of large temperature gradients in process described above, we can expect to have residual stresses in the fiber due to CTE mismatch. The questions that arise are: What is the magnitude of residual strain? Has any material plastically deformed? Was work hardening initiated in the copper or silver? Did buckling occur in the fiber? To gain a preliminary understanding about the physical phenomena that are taking place, a literature review was conducted and finite element simulations of the design were pursued. To simplify things, we began by studying the thermoelastic deformation response of a single bi-layer with different linear elastic material properties.

A simplified finite element model was created using ANSYS Workbench 11 to observe the deformation of a copper/fiber bonded structure. A state of plane stress was assumed and the in-plane dimensions of each layer were made the same size. Figure 5.1, illustrates the geometry and boundary conditions applied to the model. ANSYS Plane rry elements were used in this analysis. Planer7y is an 8-noded thermal element with one degree of freedom at each node (temperature). It is suitable for 2-D steady-state or transient analysis. Plane $77^{7}$ also has orthotropic material properties capabilities [28]. The reference temperature was set to $\left(50^{\circ} \mathrm{C}\right)$ and the structure was defined with a temperature of $0^{\circ} \mathrm{C}$ at each node. This was done to observe the structural response to the cooling process. As shown in Fig. 5.2, we observe an 


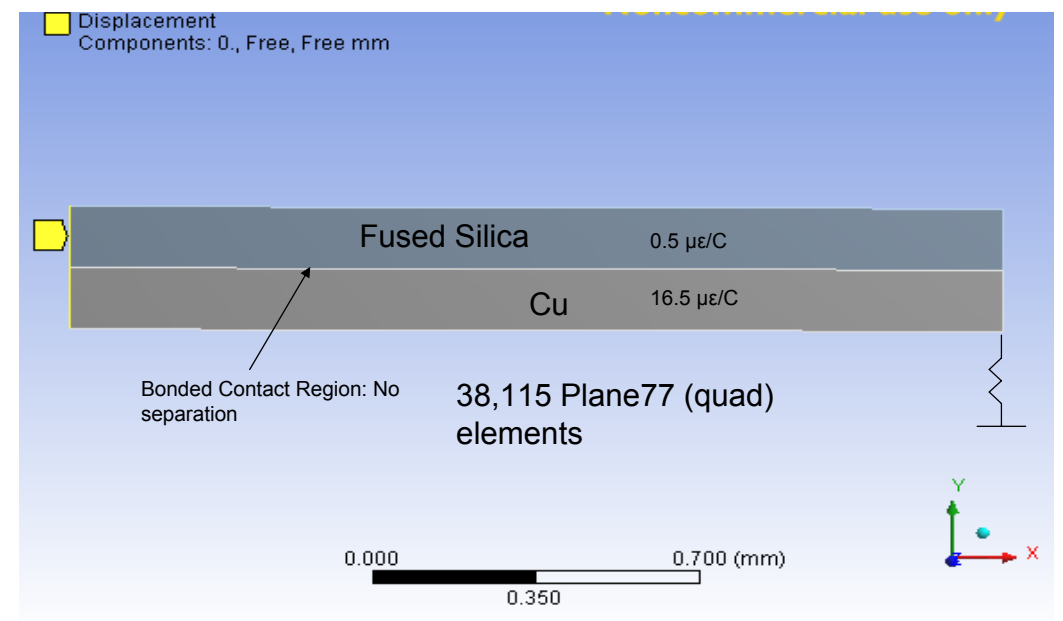

Figure 5.1: The dimensions of each layer are $\mathrm{L}=2 \mathrm{~mm}$, and $\mathrm{h}=0.15 \mathrm{~mm}$. $E_{\text {copper }}$ $=110 \mathrm{GPa}, \nu_{\text {copper }}=0.34, E_{\text {fiber }}=72.4 \mathrm{GPa}, \nu_{\text {fiber }}=0.2$. The contact region is defined as bonded, weak springs have been added to prevent rigid body motion.

effective actuation device and can see that a large amount of bending and midplane strain occurs.

The structural deformation of multilayer materials is well documented in literature, [39, 40, 38, 41], as these types of structures are used in MEMS systems. When layered plates, of comparable thickness and of different material properties, are subjected to temperature change, two main structural deformations occur; straining of the midplane and bending [40], agreeing with the results shown in Fig. 5.2. Typically, the average curvature developed as a function of temperature is used to characterize the deformation response. There are three deformation regimes that have been identified in this characterization. The first regime involves a linear relationship be- 


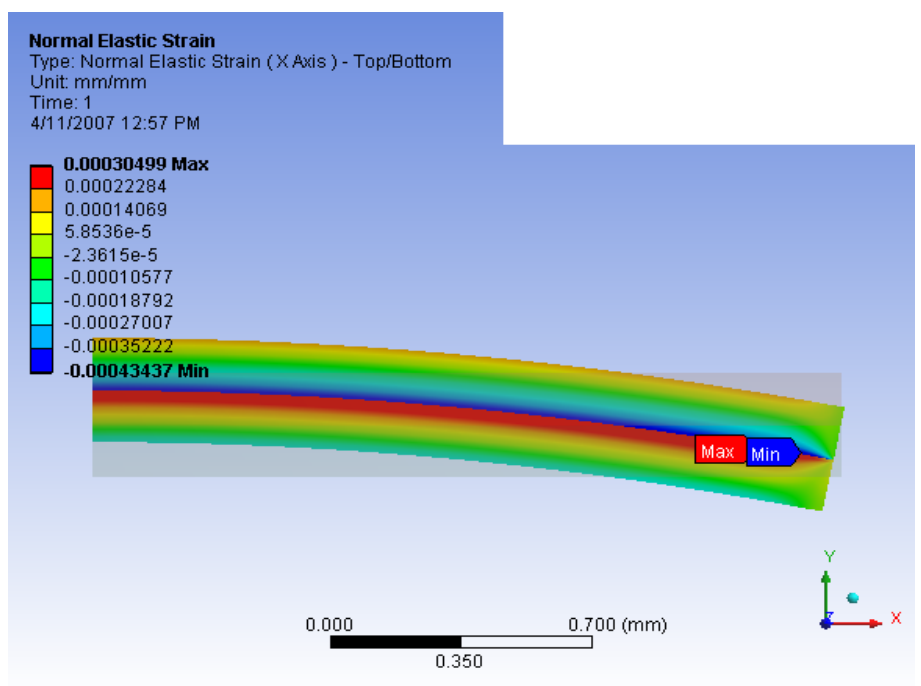

Figure 5.2: Resulting deformation and axial strain state from model described in Fig. 5.1

tween the average curvature and temperature, where conventional thin-plate theory adequately describes the deformation. The second regime consists of a nonlinear relation, due to geometric nonlinearity, between the curvature and temperature and still exhibits spherical symmetric curvature $\left(\kappa_{x}=\kappa_{y}\right)$. The third regime begins when the deformation response bifurcates from spherical to ellipsoidal deformation $\left(\kappa_{x} \neq \kappa_{y}\right)$, in the material's effort to relieve its midplane strain [40]. In general bifurcation is dependent on the size of the plate in question and as its in plane dimensions grow larger, the temperature at which bifurcation occurs decreases. Additional deformations regimes exist when material nonlinearities are present and are discussed in $[39]$. 
The reliability of our sensor at sustained high temperatures is another concern. Dunn, et al. [38], observed both creep and stress relaxation in thin-film bilayers at isothermal holds. They note that when one or more layers are metallic, the deformation of the structure can be significantly altered due to combined action of creep and stress relaxation especially at high temperatures. Previous research has shown that stress relaxation is most pronounced during the early stages of an isothermal hold. Also, for many metal thin film systems, significant stress relaxation can occur over a couple of hours and at temperatures as low as $100^{\circ} \mathrm{C}$. A question left to answer is what is the state of the fiber after the initial cooling, i.e. large residual strain, buckled, and whether this state will be relaxed due to nonlinear phenomena such as creep or stress relaxation. Dunn, et al. [38], showed a case where initial cooling caused buckling in a plate and creep/stress relaxation acted to unbuckle the plate during an isothermal hold. These questions need to be answered in order to further the development of this sensor.

\subsubsection{Conclusions}

High temperature sensing is currently in high demand. Fiber-optic methods offer many advantages over other systems. However the road to distributed sensing of pressure at high temperatures possess the same problem met by other actuators and 
sensors and that is unwanted deformations due to misfit strains, commonly caused by thermal CTE mismatch. In order to make the sensor design described in this paper a reality, a more robust bonding strategy is needed. An obvious start would be to select materials that have high temperature sustainability and similar CTE values to the fiber, thus reducing the magnitude of the misfit strain. A materials search was conducted using the CES software. The CES software contains 2,699 different materials with a detailed list of their properties. Information for each material includes values such as: composition, mechanical, thermal, and electrical properties, durability, geo-economic data, energy and emissions, and useful notes [42]. Materials include: ceramics and glasses, hybrids (composites, foams, natural materials), fibers and particulates, and metals. The graphing feature of CES was used to discover the materials which have a comparable CTE value to fused silica (fiber material) and whose working temperature was above $800^{\circ} \mathrm{C}$. Typically, metals, ceramics and glasses can withstand high temperatures, thus two separate searches with above stated criteria was done for metal, and ceramics/glasses. Figure 5.3 shows the results from the metals material database.

Notice from Fig. 5.3, at temperatures exceeding $800^{\circ} \mathrm{C}$ there are no metals with CTE's between 0.5-2 $\frac{\mu s t r a i n}{{ }^{\circ} \mathrm{C}}$. The next closest materials are Molybdenum Alloy 366, Tungsten-2\% Nickel-1\%Iron and Tantalum-10\% Tungsten Alloy. Of these three ma- 


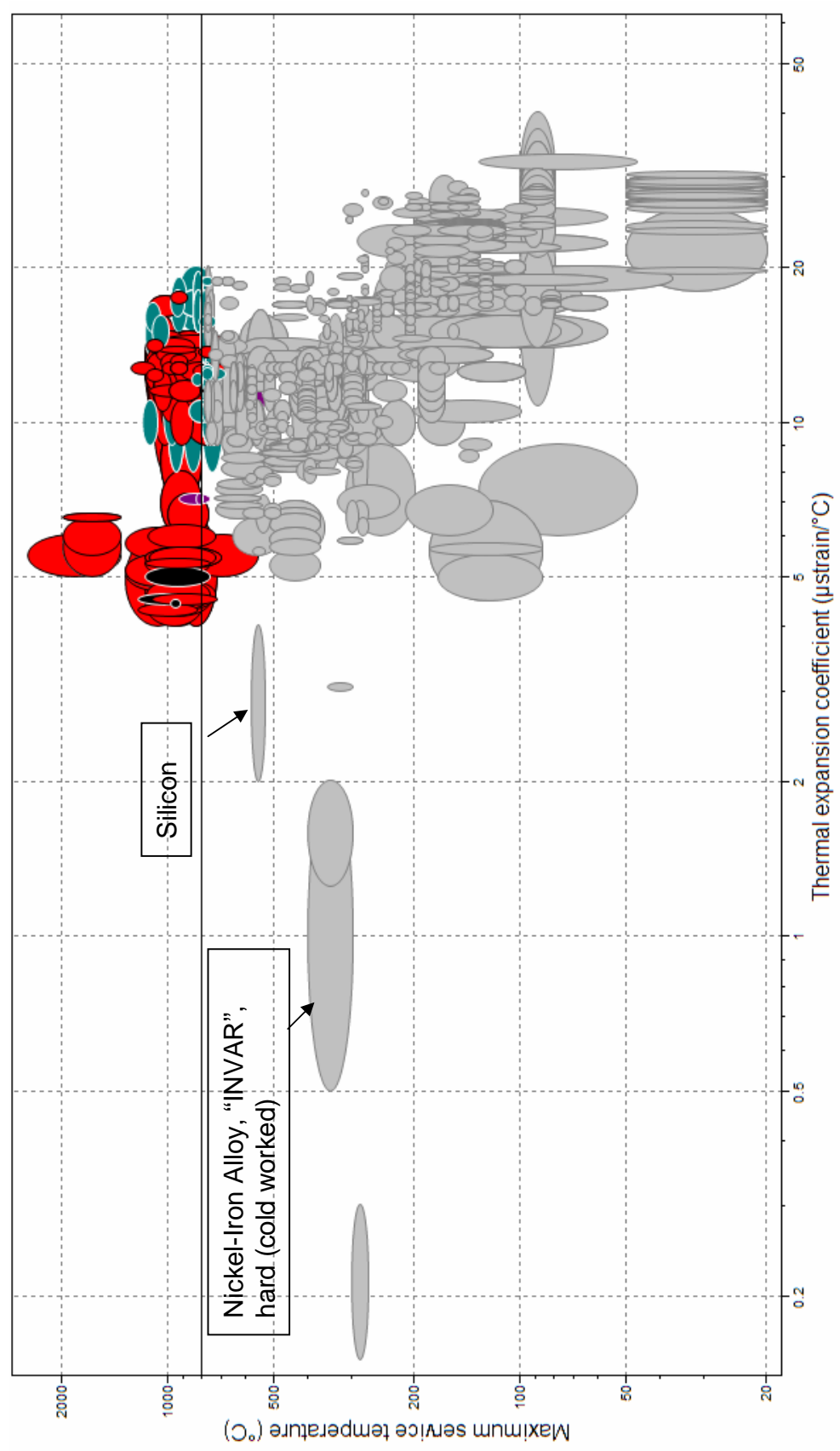

Figure 5.3: Material Search (Metals), Maximum Service Temperature vs. CTE 


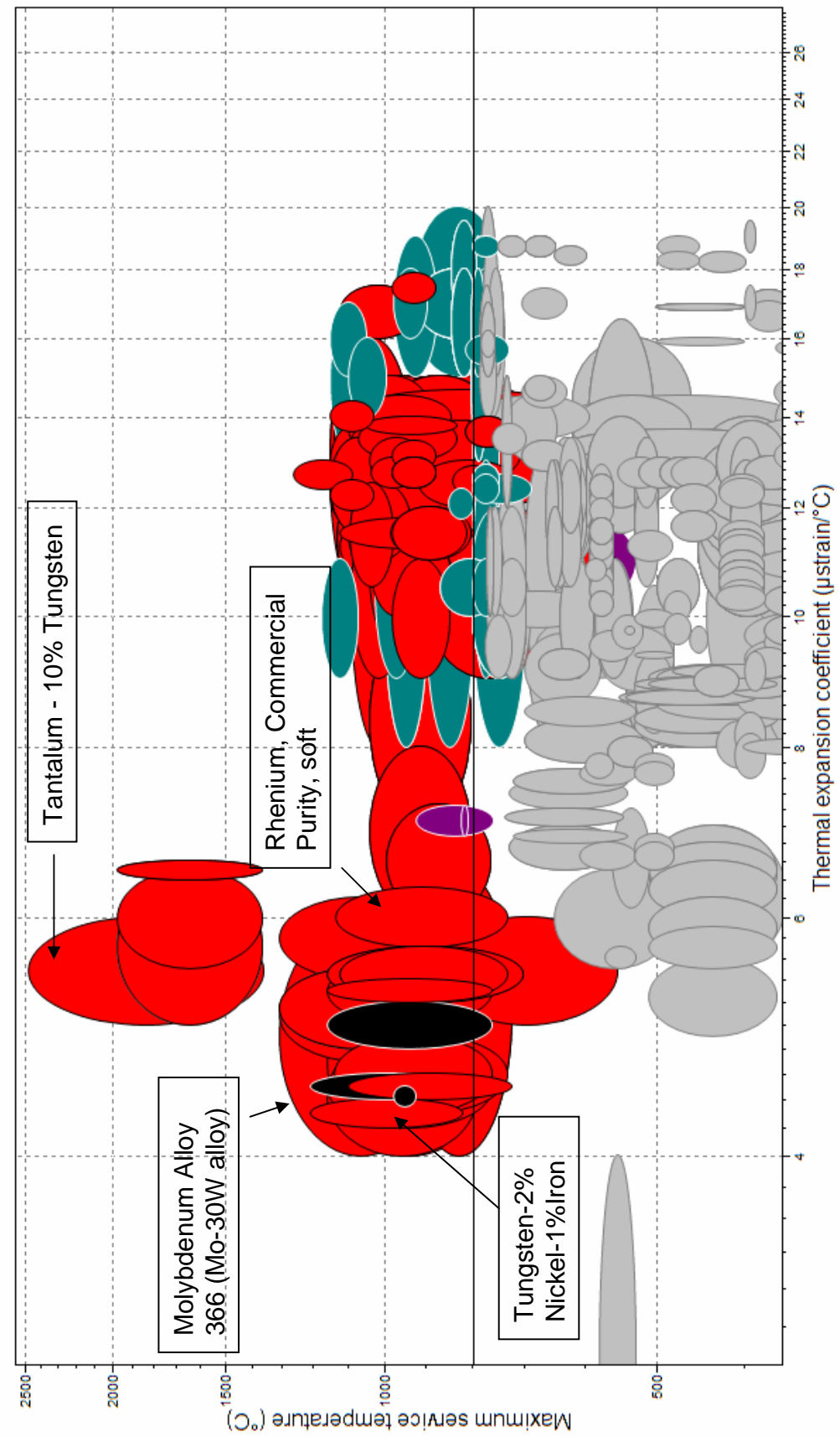

Figure 5.4: Material Search (Metals), Figure 5.3 zoomed in, with color rendering turned off. 
Table 5.1: Material Properties

\begin{tabular}{|c|c|c|}
\hline & Stainless Steel AISI 316L & Tantalum-10\% Tungsten Alloy \\
\hline Young's Modulus (GPa) & $190-205$ & $195-215$ \\
\hline Poisson's Ratio & $0.265-0.275$ & $0.33-0.36$ \\
\hline Yield Strength $(\mathrm{MPa})$ & $170-310$ & $380-560$ \\
\hline Max. Service Temp. $\left({ }^{\circ} \mathrm{C}\right)$ & $750-925$ & $1367-2482$ \\
\hline Melting point $\left({ }^{\circ} \mathrm{C}\right)$ & $1375-1400$ & $3030-3040$ \\
\hline Fatigue Strength at $10^{7}$ cycles $(\mathrm{MPa})$ & $256-307$ & $300-400$ \\
\hline CTE $\left(\frac{\mu s t r a i n}{{ }^{\circ} \mathrm{C}}\right)$ & $15-18$ & $5-6$ \\
\hline
\end{tabular}

terials, Tantalum-10\% Tungsten Alloy is most appealing because it shares similar mechanical material properies to that of stainless steel. In contrast, Molybdenum Alloy 366 and Tungsten-2\% Nickel-1\%Iron are very stiff materials (Young's modulus $>340 \mathrm{GPa}$ ), and will not work well for diaphragm based sensors. Table 5.1 compares some important material properties to diaphragm based sensor designs between Tantalum-10\% Tungsten Alloy and stainless steel.

For our purposes, Tantalum-10\% Tungsten Alloy shows much better characteristics over stainless steel: higher strength, maximum operating temperature and most importantly a three times lower CTE value. The major draw back of Tantalum is its cost (518.4-829.4 USD/kg) [42].

Figure 5.5 shows the maximum service temperature versus coefficient of thermal expansion for ceramics and glasses. There are many materials which fall into our 
desired range, however since they are mostly a ceramic material, high stiffness and low strength characteristics, they are not useful for our purposes.

Beyond the material selection, the exact behavior of the bonded structure at working temperatures needs to be identified. Buckling analysis of the fiber due to thermal loading as well as thermal cycling experiments at high temperatures, where the deformation of the diaphragm and the bond are locally monitored, may lead to some interesting realizations and designs. 


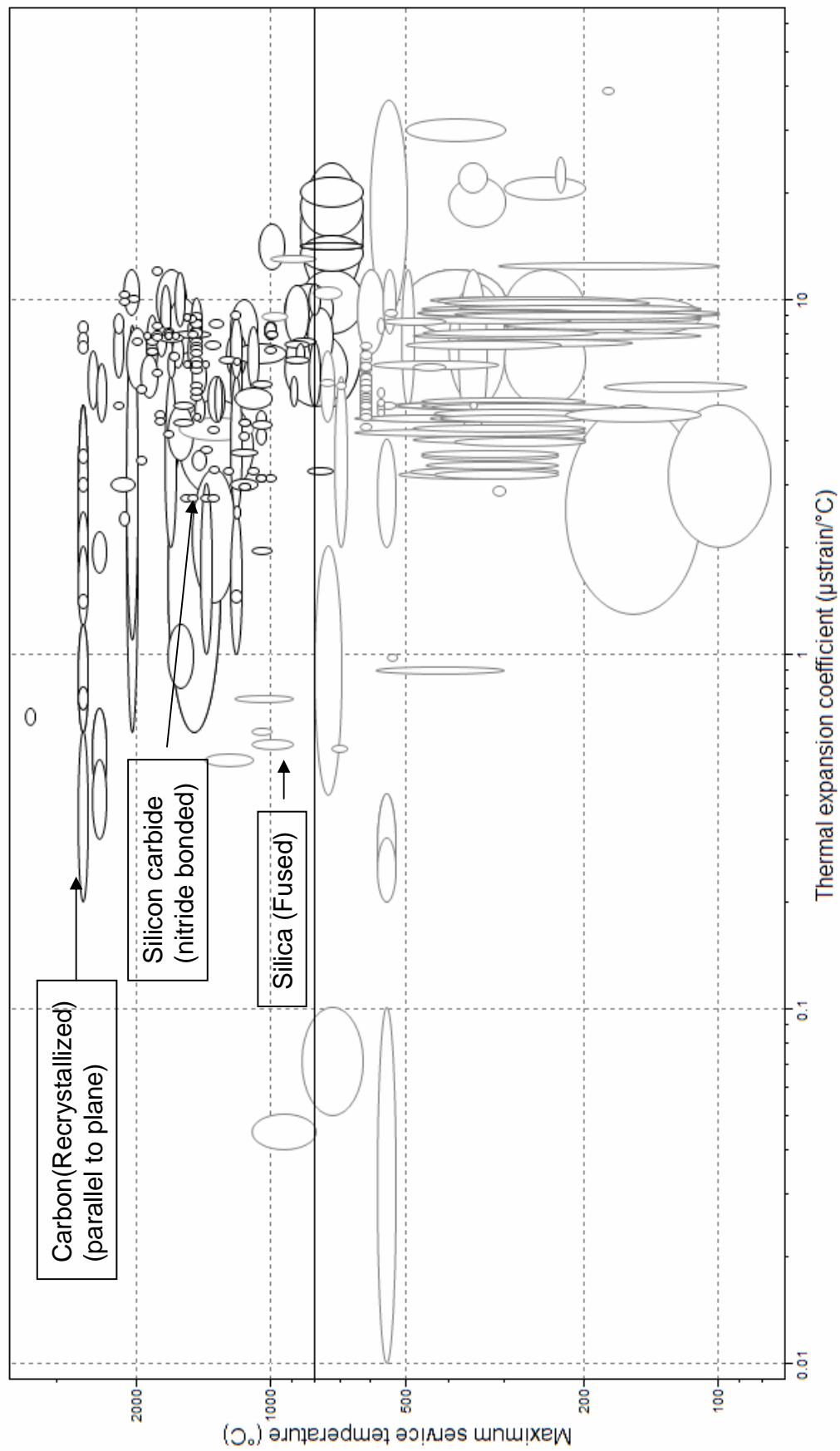

Figure 5.5: Material Search (Ceramic and glasses), Maximum Service Temperature vs. CTE 


\section{Bibliography}

[1] M.E. Palmer, M.A. Davis, G.P. EngelBrecht, R.S. Fielder, Wing Ng, Bo Song, and Aditya Ringshia. Un-cooled Fiber-Optic Pressure Sensor for Gas Turbine Engines, Operation to $1922^{\circ} \mathrm{F}$ and 500psig. In 44th AIAA Aerospace Sciences Meeting and Exhibit, Reno, Nevada, 2006.

[2] http://www.intersema.ch/site/applic_automotive.php.

[3] http://www.uscg.mil/hq/g-cp/cb/aug2000/deep.html.

[4] http://www.newscientist.com/article.ns?id=dn4715.

[5] H. Xiao. Self-Calibrated Interferometric/Intensity Based Fiber Optic Pressure Sensors. PhD thesis, Virginia Polytechnic Institute and State University, Blacksburg, VA, 2000.

[6] Duane Tandeske. Pressure Sensors Selection and Application. Marcel Dekker, INC, 270 Madison Ave. New York, NY 10016, 1991. 
[7] G. Fowles. Flow, level and pressure measurement in the water industry. Butterworth-Heineman Ltd, 1993.

[8] C. Linder, T. Tschan, and N.F. deRooij. Deep dry etching techniques as a new IC compatible tool for silicon micromachining. In Procedding of Transducers'91, 1991.

[9] Juncheng Xu. High Temperature High Bandwidth Fiber Optic Pressure Sensors. PhD thesis, Virginia Polytechnic Institute and State University, Blacksburg, VA, 2005.

[10] R. Puers. Capacitive sensors: When and how to use them. Sensors and Actuators A, 37:93-105, 1993.

[11] B. Diem, P. Rey, S. Renard, S. Viollet Bosson, H. Bono, F. Michel, M.T. Delaye, and G. Delapierre. SOI 'SIMOX'; from bulk to surface micromachining, a new age for silicon sensors and actuators. Sensors and Actuators A, 46:8-16, 1995.

[12] Gerhard H. Kroetz, Martin H. Eickhoff, and Helmut Moeller. Silicon compatible materials for harsh environment sensors. Sensors and Actuators A, 74:182-189, 1999.

[13] L. Michalski, K. Eckersdorf, J. Kucharski, and J. McGhee. Temperature Measurement. John Wiley \& Sons, West Sussex PO19 1UD, England, 2000. 
[14] D.A. Krohn. Fiber Optic Sensors Fundamentals and Applications. Instrument Society of America, Research Triangle Park, NC 27709, 2000.

[15] J.W. Berthold. Historical review of microbend fiber-optic sensors. Journal of Lightwave Technology, 13:1193-1199, 1995.

[16] Robert S. Fielder, Roger G. Duncan, Carrie L. Kozikowski, and Matthew T. Raum. High-Temperature Fiber Bragg Grating Sensor Optimization for 3D Temperature Mapping of the SAFE-100A Thermal Simulator. In Proceedings of the Space Nuclear Conference, San Diego, California,, 2005.

[17] A.D. Kersey, M.A. Davis, H.J. Patrick, M. LeBlac, K.P. Koo, C.G. Atkins, M.A. Putnam, and E.S. Frieback. Fiber Grating Sensors. Journal of Lightwave Technology, 1:1442-1463, 1997.

[18] Sriraman Kannan, Jerry Z, Y. Guo, and Paul J. Lemaire. Thermal Stability Analysis of UV-Induced Fiber Bragg Gratings. Journal of Lightwave Technology, $15,1997$.

[19] U. Sennhauser, A. Frank, P. Mauron, and Ph. M. Nellen. Reliability of optical fiber bragg grating sensors at elevated temperature. In Proceedings of 38th Annual 2000 IEEE International Symposium on Reliability Physics, 2003.

[20] http://www.ivgfiber.com. 
[21] M.G. Xu, J.L. Archambault, L. Reekie, and J.P. Dankin. Discrimination Between Strain and Temperature Effects using Dual Wavelength Fiber Grating Sensors. Elec. Lett., 30:1085, 1994.

[22] Klaus Kasten, Jörg Amelung, and Wilfried Mokwa. CMOS-compatible capacitive high temperature pressure sensors. Sensors and Actuators A, 85:147-152, 2000.

[23] M. Eickhoff, P. Reinhardt, and G. Kroetz. Accelerated Aging of Micromachined Silicon/Silicon Carbide Diaphragms by the Application of Pressure and Temperature. In 2nd Micro Materials, Berlin, 1997.

[24] Alexander A. Ned, Robert S. Okojie, and Anthony D. Kurtz. 6H-SiC Pressure Sensor Operation t $600^{\circ} \mathrm{C}$. In IEEE 1998, 1998.

[25] X. Fang, R.G. May, A. Wang, and R.O. Claus. A fiber-optic high-temperature sensor. Sensors and Actuators A, 44:19-24, 1994.

[26] S. Dasgupta, J.S. Scofield, and J.T. Boyd. Design and Fabrication of Monolithically Integrated Pressure and Temperature Sensor Using MEMS Processes for Wide Band Gap Materials. In AIAA Space 2001 Conference and Exposition, Albuquerque, NM, 2001. 
[27] Irving H. Shames and Clive L. Dym. Energy and Finite Element Methods in Structural Mechanics. Taylor and Francis, New York, 2003.

[28] ANSYS release notes 10.0.

[29] Maron et al. Multi-Parameter Fiber Optic Sensor for Use in Harsh Environments. United States Patent No. 5,892,860, 1999.

[30] Ramos et al. Methods and Apparatus for Mechanically Enhancing the Sensitivity of Longitudinally Loaded Fiber Optic Sensors. United States Patent No. 6,246,048 B1, 2001.

[31] Bailey et al. Fluid Diffusion Resistant Glass Encased Optical Sensor. United States Patent No. 6,877,378 B2, 2005.

[32] J. N. Reddy. An Introduction to Nonlinear Finite Element Analysis. Oxford University Press, Oxford, UK, 2004.

[33] William P. Eaton, Fernando Bitsie, James H. Smith, and David W. Plummer. A new analytical solution for diaphragm deflection and its application to a sufacemicromachined pressure sensor. In International Conference on Modeling and Simulation of Microsystems, 1999. 
[34] W. A. Nash and I. D. Cooley. Large Deflections of a Clamped Elliptical Plate Subjected to Uniform Pressure. Journal of Applied Mechanics, 26:291-293, 1959.

[35] S. Timoshenko. Theory of Plates and Shells. McGraw Hill, New York, NY 10020, 1940.

[36] Ephraim Suhir. Structural Analysis in Microelectronic and Fiber-Optic Systems. Van Nostrand Reinhold, New York, NY 10003, 1991.

[37] ANSYS workbench release notes 10.0.

[38] Zhang Yanhang and Martin L. Dunn. Geometric and material nonlinearity during the deformation of micron-scale thin-film bilayers subject to thermal loading. Journal of the Mechanics and Physics of Solids, 52:2101-2126, 2004.

[39] M. Finot and S. Suresh. Small and Large Deformation of Thick and ThinFilm Multi-Layers: Effects of Layer Geometry, Plasticity and Compositional Gradients. Journal of the Mechanics and Physics of Solids, 44:683-721, 1996.

[40] Zhang Yanhang, Martin L. Dunn, and Victor M. Bright. Deformation and Structural Stability of Layered Plate Microstructures Subjected to Thermal Loading. Journal of Microelectromechanical Systems, 11:372-384, 2002.

[41] Ken Gall, Zhang Yanhang, Martin L. Dunn, and Brian A. Corff. Thermal cycling 
response of layered gold/polysiliconMEMS structures. Journal of Mechanics of Materials, 36:45-55, 2004.

[42] CES online notes 2006.

[43] J.N. Reddy. An Introduction to the Finite Element Method. McGraw Hill, New York, NY 10020, 2006.

[44] Saad A. Ragab. An Introduction to the Finite Element Method Class Notes. Virginia Polytechnic Institute and State University, Blacksburg, VA, 2002. 


\section{Appendix A}

\section{Assembly Details of Bellows}

\section{Design}

The materials are as follows, refer to Fig. 2.5: Stainless steel rod, 0.020"O.D., 0.014"

I.D., Pyrex glass capillary tube, 0.236" (6mm) O.D., 0.197" (5mm) I.D., and 0.25" stainless steel type 303 rod stock was all purchased from the Small Parts Catalog (SMC). The Nickel bellows were given to us, free of charge, by Servometer. A drawing of both the bellows and stainless steel hub are given in the bellow figures. Notice that in drawing b, there are two off-center holes called out. These are the fiber exit holes, thus there will be four fiber ends exiting the sensor. This will allow a safety net, should one of the fibers break during instrumentation. 


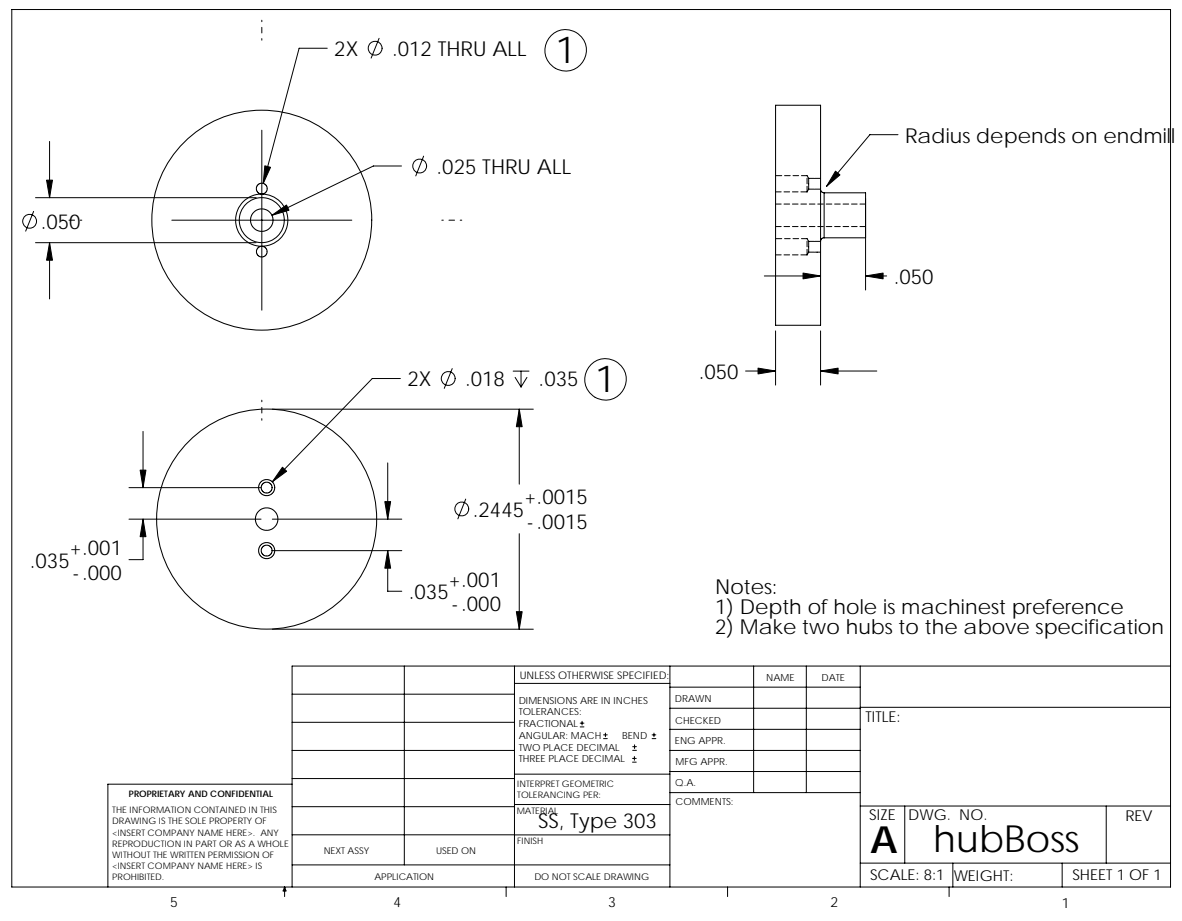

Figure A.1: Detailed Drawing of the Hub

A detailed assembly of the sensor prototype is as follows. First the pieces were to be cut to length. The glass tube was cut to length using a diamond saw cutter located at Luna Innovations. The first step in the assembly procedure was to tack weld one of the hubs to the stainless steel rod using the micro lathe welding tool at Luna Innovations. Next, the fibers were to be bonded into place. Along the top of the rod, a fiber consisting of Bragg gratings was attached to the stainless steel rod at three 


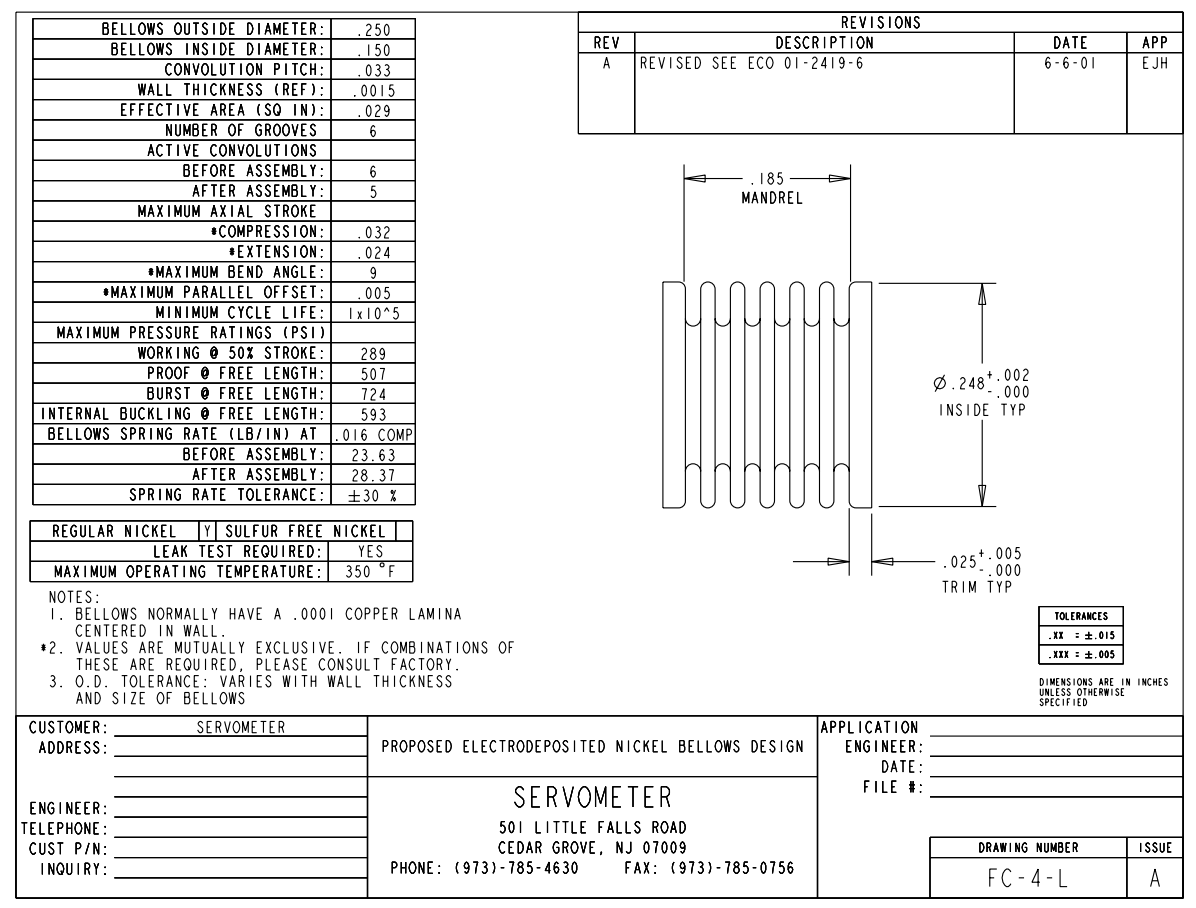

Figure A.2: Detailed Drawing of the Bellows 
points using epoxy EPY 150, a product by BLH, Vishay Measurements Group. In other words, three gratings were attached to the rod, one in the center and the other two near the hubs. Along the bottom of the rod, a fiber without Bragg gratings was attached to the rod using the same epoxy. This fiber was attached throughout the available length between the hubs. The exiting fibers were then sealed using EPY 500, a Vishay Measurements group product. Next, a bellows was attached via EPY 500 epoxy, to the fixed hub and allowed time for curing. Meanwhile the other bellows was attached, by the same method, to the glass tube and also cured for twenty four hours. The final steps involved attaching the free end of the glass tube to the fixed bellows with EPY 500. Next the second hub was ready to be put into place. First the fibers were fed through the free hub, then the hub was attached via EPY 500 to the bellows and finally the structure was placed in moderate compression and tack the hub boss portion was tack welded to the stainless steel rod. The very last step involved sealing both welds and the remaining fiber exit holes with EPY 500. 


\section{Appendix B}

\section{Bellows Testing Procedure}

An Ametek pneumatic pressure regulator, i.e. dead weight calibrator, was used to regulate the pressure. Because the dead weight calibrator can only regulate pressure manually, only one test cycle was preformed. The output of the dead weight calibrator connects to a Swagelok T-fitting. On one branch a low pressure needle gauge, made by Omega, was attached to give further confidence in the pressure being regulated. The other branches were input from the dead weight calibrator and the output went to a port in the pressure vessel. One port on the pressure vessel was equipped with another needle pressure gauge for verification of the pressure inside. The rest of the ports were sealed with a plug. One end cap allowed for fiber exit to the Distributed Sensing System (DSS) an in-house fiber signal processing unit, and 
the other end allowed for a K-type temperature transducer to be placed just above the sensor. Note that in this experiment the response of the fiber bragg grating was monitored using the DSS. The Pressure valve on the Nitrogen tank only allowed a maximum of 60 psi to be discharged to the input of the dead weight calibrator. Thus, the test was modified for one cycle (up and down) of the pressure changing by increments of 5psi every 5 minutes from 0-55psi. Every 5 minutes the appropriate weight was added or subtracted to the main shaft of the dead weight calibrator to regulate the desired temperature. The test started at approximately 13:36 pm and ended at 15:43 pm. The test began with increasing the pressure to 10 psi from zero, because in previous testing lower pressure resulted in no response. 


\section{Appendix C}

\section{Brief Review of the Structural}

\section{Concepts Used in Ch. 2}

\section{C.1 Strain}

Before introducing the sensor design, a short introduction to the concept of strain is presented to help the reader. Strain is a dimensionless quantity used to quantify the deformation of a body under some loading condition or stress state. Consider the one-dimensional case of a rod of length, L, fixed at one end and being pulled by 
some force, F, at the other end. The resulting strain is defined as:

$$
\epsilon=\frac{\dot{L}-L}{L}
$$

Where, $\dot{L}$ is the new measured length of the rod. Applying this concept to a differential element of length, $\Delta x$ and taking the limit as this length approaches zero yields,

$$
\epsilon=\lim _{\Delta x \rightarrow 0} \frac{\Delta u}{\Delta x}=\frac{d u}{d x}
$$

Here, $\Delta u$ is the change in length of the differential element due to some external loading. Similarly, in three dimensions, strain is defined as a second order tensor with nine components, it can be shown that the strain matrix is symmetric [27], thus only six strain terms are needed to define the strain-state of a body:

$$
\begin{aligned}
\epsilon_{x x}=\frac{\partial u_{x}}{\partial x} & \epsilon_{x y}=\frac{1}{2}\left(\frac{\partial u_{x}}{\partial y}+\frac{\partial u_{y}}{\partial x}\right) \\
\epsilon_{y y}=\frac{\partial u_{y}}{\partial y} & \epsilon_{y z}=\frac{1}{2}\left(\frac{\partial u_{y}}{\partial z}+\frac{\partial u_{z}}{\partial y}\right) \\
\epsilon_{z z}=\frac{\partial u_{z}}{\partial z} & \epsilon_{x z}=\frac{1}{2}\left(\frac{\partial u_{x}}{\partial z}+\frac{\partial u_{z}}{\partial x}\right)
\end{aligned}
$$

$x, y$ and $z$ are in reference to a right-handed Cartesian reference frame. 


\section{C.1.1 A Note About the Compatibility Equations}

From the six strains terms, for a given displacement field one can easily compute the strain matrix. In contrast, for a given strain matrix, one must obtain the displacement field by solving six partial differential equations. In order to ensure a single-valued, continuous displacement field, restrictions must be imposed on $\epsilon_{i j}$. These restrictions are known as the compatibility equations [27]. It turns out that there are 81 equations of compatibility, only six of which are independent.

\section{C.2 The Finite Element Method}

The finite element method is a numerical method used to solve complicated governing equations with sometimes abstruse associated boundary conditions. In this method the problem domain is viewed as a collection of subdomains (finite elements), and in these subdomains the governing equations are approximated by any traditional variational method, Ritz, Galerkin, collocation, etc... [43]. The motivation being that it is easier to represent a complicated function by a collection of simple polynomials. In most approximate methods, the primary variable is approximated by the following linear combination of appropriately chosen approximation functions $\phi_{j}(x)$ 
and unknown coefficients, $c_{j}$ :

$$
u(x) \approx U_{N}(x)=\sum_{j=1}^{N} c_{j} \phi_{j}(x)
$$

Here, $\mathrm{u}$ represents the exact solution to a particular differential equation and associated boundary conditions, and $U_{N}$ represents the approximation of the primary variable. $U_{N}$ is completely known once $c_{j}$ is known. $\phi_{j}(x)$ is chosen such as to satisfy the essential boundary conditions of the problem. Consider solving the second-order differential equation, an equation common to many engineering fields :

$$
-\frac{d}{d x}\left[a(x) \frac{d u}{d x}\right]=f(x)
$$

Substituting Eq. C.6 into Eq. C.7

$$
-\frac{d}{d x}\left[a(x) \frac{d U_{N}}{d x}\right]=f(x)
$$

Since we are using an approximation for the primary variable, the left-hand side of the above equality will be not be equal to the right-hand side, thus, there will be a residual, $R\left(x, c_{j}\right)$ :

$$
R\left(x, c_{j}\right)=-\frac{d}{d x}\left[a(x) \frac{d U_{N}}{d x}\right]-f(x)
$$


Some variational methods seek to find $\mathrm{N}$ equations among $c_{j}$ to make $R\left(x, c_{j}\right)$ equal to zero. Since this cannot happen at every point, the weighted-residual method is used, which requires $R\left(x, c_{j}\right)$ to vanish in a weighted-residual sense [43]:

$$
\int_{0}^{L} w_{i}(x) R\left(x, c_{j}\right) d x=0 \quad(i=1,2 . . N)
$$

Where, $w_{i}$ are the linearly independent weight functions. Different methods define different weight functions, the Galerkin method for instance, defines $w_{i}=\phi_{i}$. The weak formulation also provides a means of classifying the boundary conditions into natural and essential types. An essential boundary condition specifies the primary variable $(\mathrm{u})$ at the boundary, while a natural boundary condition specifies the secondary variable $\left(Q=p \frac{d u}{d x}\right)$ at the boundary. Note, that the term weak refers to the transfer of the differentiation from the dependent variable to the weight function such that all natural boundary conditions are satisfied [43], as will be shown below.

\section{C.2.1 Development of the Weak Form}

First we start with the weighted integral statement Eq. C.10, yielding for Eq. C.7

$$
\int_{0}^{L} w_{i}(x)\left[-\frac{d}{d x}\left[a(x) \frac{d u}{d x}\right]-f(x)\right] d x=0
$$


To split the differentiation between, $\mathrm{w}$ and $\mathrm{u}$, integration by parts on the first term must be employed, yielding:

$$
\int_{0}^{L}\left(a \frac{d w}{d x} \frac{d u}{d x}-w f\right) d x-\left[w a \frac{d u}{d x}\right]_{0}^{L}=0
$$

In the weak formulation, the weight function has the meaning of a virtual change of the primary variable, $w \approx \delta u$. So if an essential boundary condition (one that specifies the primary variable at the boundary) is specified, then the virtual change (weight) is zero at that boundary. So for the boundary conditions, $u(0)=u_{0}$ and $\left(a \frac{d u}{d x}\right)_{x=L}=Q_{L}$, the above equation becomes:

$$
\int_{0}^{L}\left(a \frac{d w}{d x} \frac{d u}{d x}-w f\right) d x-w(L) Q_{L}=0
$$

Notice that the finalized weak form is composed of a bilinear symmetric $(\mathrm{B}(\mathrm{w}, \mathrm{u}))$ and linear $(\mathrm{l}(\mathrm{w}))$ term, and thus can be rewritten as:

$$
\begin{aligned}
B(w, u) & =l(w) \\
B(w, u) & =\int_{0}^{L} a \frac{d w}{d x} \frac{d u}{d x} d x \\
l(w) & =\int_{0}^{L} w f d x+w(L) Q_{L}
\end{aligned}
$$




\section{C.2.2 Assembly of Finite Elements}

As mentioned above, the finite element method breaks the problem domain into subdomians (finite elements). The solution to the governing mathematical model is approximated over each finite element, using a polynomial approximation of the form:

$$
U_{h}^{e}=\sum_{j=1}^{n} u_{j}^{e} \psi_{j}^{e}(x)
$$

where, $U_{h}^{e}$ are the values of the solution $\mathrm{u}(\mathrm{x})$ at the nodes of the element, and $\psi_{j}(x)$ are the approximation functions over the element. The choice of the use of a polynomial approximation, in this case, helps allow for easy integration, along with presenting access to interpolation theory, thus helping to develop the approximation functions. The approximate solution must satisfy three conditions in order to be convergent to the actual solution $\mathrm{u}$. First it should be continuous over the element and differentiable, the number of times stem as required by the weak form. Secondly, it should be a complete polynomial, that is include all lower order terms up to the highest. This is to ensure that all the possible states will be captured in the solution. Lastly, the values at nodal junctions need be the same, that is, continuity of the primary variable at points common to elements must be enforced. 
After the domain is broken into elements (finite element mesh), the elemental equations are formed, and then the system is assembled into one matrix equation. This process begins by solving for the approximate primary variable locally, i.e. at each node of an element. Thus, the weak form of the problem is defined for each element. To illustrate this consider again, a second order differential equation of the form:

$$
-\frac{d}{d x}\left[p(x) \frac{d u}{d x}\right]+q(x) u-f(x)=0
$$

Recall that the weak form formulation of a second order differential equation contains only the first derivative of the unknown function, hence it requires continuity of the variable $\mathrm{u}(\mathrm{x})$ only. In general, if the weak form of your problem has $\frac{d^{m} u}{d x^{m}}$, it will require continuity of $\frac{d^{m-1} u}{d x^{m-1}}$ down to $\mathrm{u}(\mathrm{x})$. In the problem under consideration we can use a linear element defined by two nodes, $x_{1}^{e}, x_{2}^{e}$. Here e, is used to define the element number. The weak form over the element is then [44]:

$$
\int_{x_{1}^{e}}^{x_{2}^{e}}\left(\frac{d w}{d x} p \frac{d u}{d x}+w q u\right) d x=\int_{x_{1}^{e}}^{x_{2}^{e}} w f d x+w\left(x_{1}^{e}\right) Q_{1}^{e}+w\left(x_{2}^{e}\right) Q_{2}^{e}
$$

where, $Q_{1}^{e}=-\left(p \frac{d u}{d x}\right) x_{1}^{e}$ and $Q_{2}^{e}=-\left(p \frac{d u}{d x}\right) x_{2}^{e}$ are the secondary variables at the nodes. There are no boundary conditions applied at the nodes, the boundary condi- 
tions are only applied at the first node and the last node. So at inter-element nodes the secondary variables satisfy:

$$
\begin{aligned}
& Q_{2}^{e-1}+Q_{1}^{e}=0 \\
& Q_{2}^{e}+Q_{1}^{e+1}=0
\end{aligned}
$$

However, in the case of externally applied sources of secondary variables at these nodes, additional terms will contribute to the right hand side of the above equations.

The approximation function, $\psi_{j}(x)$, also known as shape functions are used to interpolate the value of the approximated solution over an element. For the problem under consideration, $U^{e}$ needs only to be once differentiable, thus at least a linear approximation is needed.

$$
U^{e}(\xi)=c_{1}+c_{2} \xi
$$

where $\xi$ is defined as a local coordinate related to the global coordinates by $\xi=$ $\left(x-x_{1}^{e}\right) / h_{e}$, here $h_{e}$ is the element length. The conditions wanted are:

$$
\begin{array}{ll}
\xi=0: & U^{e}=U_{1}^{e} \\
\xi=1: & U^{e}=U_{2}^{e}
\end{array}
$$


Verify that this linear approximation meets the three stated criteria above. From these conditions it is easy to obtain the coefficients $c_{1}$ and $c_{2}$ and finally,

$$
\begin{aligned}
& U^{e}(\xi)=\psi_{1} U_{1}^{e}+\psi_{2} U_{2}^{e} \\
& \psi_{1}=(1-\xi) \quad \psi_{2}=\xi
\end{aligned}
$$

To obtain the element matrices we choose two linearly independent weight functions, in the Galerkin method, the weight functions are simply the shape functions. Recalling Eq. C.16, the weak form over the element becomes:

$$
\begin{aligned}
B^{e}\left(\psi_{i}, U^{e}(x)\right) & =l\left(\psi_{i}\right) \\
B^{e}\left(\psi_{i}, \psi_{1}\right) U_{1}^{e}+B\left(\psi_{i}, \psi_{2}\right) U_{2}^{e} & =l\left(\psi_{i}\right) \\
{\left[K^{e}\right]\left\{U^{e}\right\} } & =\left\{F^{e}\right\}
\end{aligned}
$$

Finally, the global system can be made by summing the equations, forming the wellknow relation:

$$
[K]\{U\}=\{F\}
$$

where $[K]$ is known as the global stiffness matrix. After the addition of the boundary 
conditions, the primary variable can be solved for in a multitude of ways. This brief presentation of the finite element method was meant to help the reader understand the basic concepts of the finite element method through an example applied to a simple one-dimensional case. 


\section{Appendix D}

\section{Files used in computations}

Below is a listing of some of the more important "codes" used in the development of this thesis. The first two files refer to the input file introduced in Chapter 3. To be used, place the first file in your ANSYS working directory and save the second as a .mac file in your working directory or in the ANSYS macro library:

C: \Program Files\Ansys Inc \v100\ANSYS \apdl\path

Then in the ANSYS black box module select file, read input from, then select the input file (ANSYS input file). Note many parameters can be changed within the input file and its function file. When the solution finishes use the $*$ status command to view the results, see [28]. The last file is an Mathematica file which calculates and plots the deflection and axial strain along the semi-major axis of an ellipse via the 
Nash/Cooley formulation discussed in Chapter 3.

- ANSYS input file

- ANSYS MACRO "path" called in input file

- Nash and Cooley elliptical plate formulation for any ellipse configuration 